\title{
ESTADO DE CONOCIMIENTO ACTUAL Y PROBLEMAS DE CONSERVACIÓN DE LOS MELOIDAE (COLEOPTERA) DE LA COMUNIDAD DE MADRID
}

\author{
M. García-París*, N. Trotta-Moreu y L. Capote
}

\begin{abstract}
RESUMEN
La recopilación de los datos publicados, acompañada de la revisión de colecciones y de observaciones de campo a lo largo de los últimos ocho años, permite registrar un total de 40 especies de la familia Meloidae en la Comunidad de Madrid. De éstas 40 especies, hay siete que únicamente se conocen a partir de capturas realizadas hace más de 25 años, y otras dos que se citan únicamente a partir de los nuevos datos de campo (Meloe rugosus y Nemognatha chrysomelina). Dos de las siete especies sin capturas recientes (Stenoria apicalis y Meloe baudueri) están representadas únicamente por uno o dos ejemplares, por lo que su presencia actual ha podido pasar desapercibida. Este no es el caso de las otras cinco (Meloe variegatus, M. cavensis, M. violaceus, Mylabris uhagonii y M. amorii), que están representadas por numerosos ejemplares en las colecciones históricas. Meloe variegatus, M. cavensis y Mylabris uhagonii, han de ser consideradas En Peligro de Extinción, o tal vez definitivamente extinguidas, si no aparecen nuevos ejemplares en un futuro próximo. Mylabris amorii sólo se ha encontrado en una localidad situada en el límite septentrional de la distribución conocida de la especie, por lo que no tenemos criterios suficientes para evaluar su situación. Meloe violaceus ha sufrido un retroceso evidente, y por lo tanto ha de considerarse como Vulnerable. En cinco especies adicionales se ha constatado una reducción evidente de la distribución actual. Esta situación se presenta en Meloe brevicollis, M. autumnalis, M. proscarabaeus, Hycleus dufourii y Mylabris dejeani. En estas especies la distribución actual se limita a zonas situadas en la Sierra, mientras que el resto de poblaciones parecen haber desaparecido por lo que deberían considerarse en la categoría de Vulnerable. Si continúa la tendencia actual, posiblemente pasarán a la categoría de En Peligro de Extinción. Meloe tuccius, una especie frecuente hace tan sólo 10 ó 15 años en el área urbana, puede considerarse como casi desaparecida y por lo tanto ha de incluirse en la categoría de Vulnerable. La imparable urbanización creciente, el uso de herbicidas en las cunetas, el empleo masivo de productos fitosanitarios en los cultivos, campos de golf y jardines y, las fumigaciones aéreas contra plagas, son en la actualidad problemas serios para las poblaciones de meloidos de Madrid.
\end{abstract}

Palabras clave: Coleoptera, Meloidae, Faunística, Conservación, Madrid, España.

* Museo Nacional de Ciencias Naturales, C.S.I.C. c/ José Gutiérrez Abascal, 2. 28006 Madrid. España. mcnp505@mncn.csic.es 


\section{ABSTRACT \\ A review of the status and conservation problems of the Meloidae (Coleoptera) in Comunidad de Madrid}

Data gathered from publications, entomological collections and field observations along the last eight years, provide information about the presence of 40 especies of the family Meloidae in Comunidad de Madrid. Seven species are only known from collections made more than 25 years ago, while two species have been found only during recent field surveys (Meloe rugosus and Nemognatha chrysomelina). Two out of the seven species without recent records (Stenoria apicalis and Meloe baudueri) are represented in Madrid by one or two specimens, so that their current presence in the region may have been overlooked. This is not the case of the other five (Meloe variegatus, $M$. cavensis, M. violaceus, Mylabris uhagonii and $M$. amorii), which are represented by numerous specimens in the historical collections. Meloe variegatus, M. cavensis and Mylabris uhagonii, must be considered at risk of extinction, but if new specimens are not found in the near future they should be treated as definitively extinct. Mylabris amorii has been found in only one locality at the northernmost edge of its known distribution range, and the evaluation of its status depends on new information. Meloe violaceus has suffered and evident decline and therefore should be treated as Vulnerable. Five additional species suffered evident reductions of their distribution ranges in Madrid (Meloe brevicollis, M. autumnalis, M. proscarabaeus, Hycleus dufourii and Mylabris dejeani). Most of the recent records for those species are restricted to mountain areas, while the lowland populations seem to have been disappeared and consequently, these species should be listed as Vulnerable. If the current tendency continues they will be transferred to the Extinction Risk level. Meloe tuccius, a frequently found species 10 to 15 years ago in the urban area of Madrid can be considerered today as nearly extinct in the area, and therefore should be treated as Vulnerable in the Region. Increasing urban development, masive use of agrochemical products in roadside works, agricultural fields, golf courses, and gardens, and aeroplane treatment of pests, represent serious current threats for the populations of meloid beetles in Madrid.

Key-words: Coleoptera, Meloidae, Faunistics, Conservation, Madrid, Spain.

\section{Introducción}

La fauna entomológica de la Comunidad de Madrid es quizás una de las mejor conocidas dentro del territorio peninsular. La actividad de varias generaciones de entomólogos residentes en Madrid, desde finales del siglo XIX hasta nuestros días, ha generado importantes colecciones, que en gran parte se encuentran ubicadas en el Museo Nacional de Ciencias Naturales (MNCN). Sin embargo, y a pesar del gran volumen de material recogido, muchos de los datos de estas colecciones históricas nunca han sido publicados. La situación es especialmente llamativa en determinados grupos en los que no ha habido especialistas ibéricos o en los que los especialistas sólo pudieron revisar una parte limitada de la colección, como es precisamente el caso que nos ocupa. En estas situaciones la información adquirida durante tantas décadas de muestreo permanece inédita y no está disponible para su utilización. Esta falta de disponibilidad supone un problema especialmente serio a la hora de la toma de decisiones para la conservación de la
Naturaleza regional. Por una parte, sin datos previos, no se puede evaluar el estado de conservación actual de una especie, ya que para determinar su estatus se requieren análisis de tendencias poblacionales, en los que por lo menos se identifique la existencia o no de cambios en su distribución geográfica, o en el tamaño de las poblaciones, a lo largo del tiempo. Por otra parte, sin datos publicados resulta casi imposible determinar en los plazos requeridos para los estudios de impacto ambiental o similares, qué especies existen en un punto geográfico concreto; y puesto que la política habitual tiende a considerar que la ausencia de información equivale a la ausencia "real" de la especie en el punto de estudio, el resultado final es que una especie sin registros publicados es una especie inexistente. Otras aplicaciones de los datos procedentes de colecciones para la identificación de áreas importantes o de especies amenazadas, han sido señaladas por numerosos autores, aunque en general, centrados en el estudio de vertebrados (por ejemplo Pleguezuelos, 1991; Shaffer et al., 1998; Martínez-Solano \& González Fernández, 2003). En 
áreas como la Comunidad de Madrid, los cambios en el uso del suelo han sido tan dramáticos en los últimos años, que en muchas de las localidades clásicas para el estudio de los insectos (como Montarco, Brunete, Villaviciosa de Odón, La Moncloa, Cerro del Telégrafo, Cerro Negro, Cerro Grande de Almodóvar...) ya resulta imposible, localizar una mínima fracción de la fauna que albergaban hace simplemente dos décadas.

Los coleópteros de la familia Meloidae no son una excepción, y la mayor parte de las localidades de captura de los ejemplares de las colecciones históricas de Madrid, incluida la del MNCN son inéditas. De los datos de la fauna de Madrid representada en esta colección únicamente se han publicado algunas citas recogidas por Rodríguez López-Neyra (1914), y las correspondientes a los representantes de las tribus Cerocomini (dos especies), Lyttini (cuatro especies) y Sitarini (tres especies), y de las tres especies madrileñas del género Hycleus (Mylabrini), que han sido el objeto de estudios previos (Capote \& García-París, 2000; García-París, 1998, 2000; García-París et al., 2003; Trotta-Moreu \& García-París, 2001). Puesto que la mayor parte del material de Meloidae de Madrid corresponde a colecciones históricas (Pérez-Arcas, Bolívar, Escalera...) presenta un valor testimonial añadido, ya que muchas de las localidades donde se recogieron especímenes han sido absorbidas por la urbe.

Los Meloidae han sido uno de los pocos grupos de insectos no dañinos para la agricultura que han atraído la atención de naturalistas desde la antigüedad. Durante el siglo XIX, en España se publicaron dos monografías sobre el grupo (Amor Mayor, 1860; Górriz Muñoz, 1882), mientras que el comercio con especies de esta familia, propiciado por sus propiedades farmacológicas, está bien documentado durante gran parte de ese mismo siglo en la Península Ibérica (Texidor Cos, 1880). Los datos geográficos publicados durante el siglo XIX e inicios del XX han sido recopilados recientemente (García-París \& Ruiz, 2005), pero a pesar de que el número de trabajos que recogen datos es relativamente elevado, el número total de citas publicadas durante ese periodo es pequeño.

Los datos disponibles sobre la distribución de las especies presentes en Madrid, además de los ya mencionados relativos a la colección del $\mathrm{MNCN}$, se encuentran dispersos en trabajos, que generalmente se refieren a un ámbito geográfico mayor (Amor Mayor, 1860; Díaz Lizana, 1864; Heyden, 1870; Marseul, 1870; Martínez Sáez, 1873; Górriz Muñoz, 1882; Sumakov, 1930; De la Fuente, 1933), o corresponden a citas puntuales publicadas en las memorias de excursiones naturalísticas (Seidlitz, 1867; Dieck, 1870; Sanz de Diego, 1880; Gómez Carrasco, 1888; Traizet, 1896; Flach, 1907). Los primeros registros publicados de Meloidae de Madrid que hemos localizado corresponden a los trabajos de Graells (1849, 1851a, 1851b, 1853), autor que además describió, entre otras, cuatro especies nuevas de Meloidae de Madrid y sus alrededores (Mylabris hieracii, Mylabris sobrina, Mylabris maculosopunctata e Hycleus dufourii). En el primer tercio del siglo XX se publican las primeras claves de identificación que incluyen a todas las especies de Madrid conocidas en la época (Rodríguez LópezNeyra, 1914), y se publican los fascículos correspondientes a la familia Meloidae del "Catálogo sistemático geográfico de los Coleópteros observados en la Península Ibérica, Pirineos propiamente dichos y Baleares" (De la Fuente, 1933), donde se recopila la mayor parte de la información corológica disponible hasta esa fecha. Estos trabajos se completan con la serie de artículos dedicados a cada tribu por Pardo Alcaide (1950, 1952, 1956a, b, 1958), a los que más recientemente siguen artículos en los que se recogen citas madrileñas concretas de varias especies (por ejemplo López-Colón, 1991; Bologna \& Aloisi, 1994; Pérez Moreno et al., 2003). Varios taxones de Meloidae han sido descritos utilizando ejemplares procedentes de territorio madrileño; algunas de estas especies se describieron con material colectado exclusivamente en Madrid como Mylabris hieracii, Mylabris sobrina, Hycleus dufourii y Euzonitis haroldi (Graells, 1849; Heyden, 1870), mientras que otras lo fueron con material de Madrid y de otras provincias del centro peninsular (Mylabris uhagonii) (Martínez Sáez, 1873).

A pesar de esta aparente diversidad de publicaciones que mencionan registros madrileños, el conjunto de citas publicadas es pequeño, y prueba de ello es que especies bien conocidas en provincias cercanas como Euzonitis sexmaculata y Nemognatha chrysomelina, o especies bien representadas por material madrileño en las colecciones históricas del $\mathrm{MNCN}$, como Meloe variegatus, nunca se han citado como presentes en la Comunidad de Madrid.

Para cubrir parcialmente este vacío, durante los últimos años hemos realizado muestreos centrados en la localización de poblaciones de Meloidae a lo largo de la mayor parte del territorio madrileño. En no pocos casos el desarrollo urbanístico nos ha impedido volver a los puntos clásicos de muestreo, mientras que en otros, las búsquedas no han resultado fructíferas, especialmente para especies del género Meloe, a pesar de una aparente buena con- 
servación de algunos de los hábitats muestreados. Estos problemas para el género Meloe (Nash, 1976; Havelka, 1980), parecen coincidir como en otros países europeos con un incremento en el uso de productos fitosanitarios que afectan sobre todo a sus hospedadores, abejas de varios géneros (Apoidea).

En este trabajo se presentan los datos bibliográficos recopilados, acompañados por los datos de la colección del MNCN y de otras colecciones públicas y privadas, que hemos tenido la oportunidad de examinar, y finalmente de los datos obtenidos en las campañas de muestreo específicas efectuadas sobre todo durante el periodo 1998-2005 en el territorio madrileño. Estos datos suponen el punto de partida a partir del cual se pueden comenzar los análisis de tendencias poblacionales, pero también, la existencia de datos históricos posibilitan una primera evaluación del estado de conservación de las poblaciones en el momento actual.

\section{Material y método}

La mayoría de las especies de Meloidae de Madrid son identificables tras el examen de la morfología externa, aunque en algunos casos se ha recurrido al examen de la genitalia masculina, especialmente en el caso del género Mylabris. La nomenclatura de los taxones tratados sigue los criterios establecidos en los catálogos de Borchmann (1917), Mader (1927) y Sumakov (1930) y sobre todo las obras de Bologna (1991) y Bologna \& Pinto (2002), con algunas excepciones referentes al mantenimiento de la tribu Sitarini, no considerada como tal por Bologna \& Pinto (2002), y a la inclusión del género Physomeloe en la tribu Lyttini, un aspecto discutido por Bologna \& Pinto (2001) y que aún está pendiente de revisión. Por otra parte estudios en curso basados en la utilización de ADN mitocondrial (García-París et al., inédito) ponen de manifiesto que algunos de los taxones que actualmente viven de Madrid deberán cambiar de nombre, por no corresponder a la especie en la que actualmente se incluyen, mientras que otros pasarán a la sinonimia de otras especies, y finalmente algunos forman parte de complejos de especies que tal vez requieran una fragmentación taxonómica. Se desconoce si estos cambios afectarán al estado de conservación ó a las distribuciones ya que por el momento los estudios filogeográficos centrados en Madrid aún no han concluido.

En algunos trabajos bibliográficos se recoge la presencia en Madrid de especies que han sido eli- minadas del catálogo de especies presentes en la Península Ibérica (García-París \& Ruiz, 2005): Mylabris geminata (Fabricius, 1798) citado en la provincia de Madrid por Graells (1853), cita que es atribuida tentativamente a Mylabris varians, única especie con diseño elitral similar encontrada en Madrid, por García-París \& Ruiz (2005). Cerocoma vahli Fabricius, 1787 citada en El Escorial y El Pardo por Rodríguez López-Neyra (1914) y de la provincia de Madrid por De la Fuente (1933), citas que en realidad corresponden a $C$. schaefferi como indicaron Pardo Alcaide (1956), Capote \& GarcíaParís (2001) y García-París \& Ruiz (2005). Mylabris (Eumylabris) fabricii Sumakov, 1924, citado de la provincia de Madrid por De la Fuente (1933), bajo la denominación de $M$. decimpunctata Petagna, 1787; como ya señalaron Pardo Alcaide (1950), Bologna (1991) y Trotta-Moreu \& GarcíaParís (2001) la mayoría de los registros ibéricos de este taxon se deben a errores de identificación con H. scutellatus (Rosenhauer, 1856). Mylabris (Eumylabris) impressa Chevrolat, 1837, citado por De la Fuente (1933) en El Escorial sin que exista ningún ejemplar como referente; siguiendo el criterio de García-París \& Ruiz (2005), no hemos tenido en cuenta este registro. Euzonitis terminata (Abeille de Perrin, 1880), fue citada por vez primera para la Península Ibérica por Rodríguez LópezNeyra (1914) como "Zonitis auricoma Escher. var. bombycina", de Aranjuez (Madrid), registro que es recogido por De la Fuente (1933). Bologna (1991, 1994), seguido por García-París \& Ruiz (2005), indica que esta cita española debe referirse a Euzonitis quadrimaculata (Pallas, 1782). Apalus bimaculatus (Linnaeus, 1761), fue citado en Madrid por Rodríguez López-Neyra (1914) a partir de un ejemplar de la colección Pérez Arcas conservada en el MNCN, pero como argumenta GarcíaParís (2000) se trata de material italiano con etiqueta errónea.

Puesto que hasta los trabajos de Bologna (1988a, 1991) los registros ibéricos de Meloe mediterraneus y de Meloe rugosus se han venido realizando bajo este último nombre, y la especie dominante en Iberia es sin embargo $M$. mediterraneus, García-París \& Ruiz (2005) asignan todas las citas ibéricas publicadas hasta 1933 con el nombre de $M$. rugosus a $M$. mediterraneus. La cita de $M$. rugosus del municipio de Madrid de Rodríguez López-Neyra (1914) y la provincial de De la Fuente (1933) se han asignado por lo tanto a M. mediterraneus, sin que por ello deba excluirse la presencia de $M$. rugosus en Madrid (ver el epígrafe correspondiente a esta especie). 
La mayor parte de los datos de colecciones provienen de la revisión de material en seco de las colecciones entomológicas del Museo Nacional de Ciencias Naturales (MNCN), del Museo de Zoología de Barcelona (Col. MZB), Museo Nacional de Ciencias Naturales de Hungría en Budapest (Col. Kaszab), Museo de Historia Natural de Trondheim, Noruega (Col. Escherich), Departamento de Biología Animal, Universidad de La Laguna (Col. P. Oromí), y las colecciones de R. Sánchez (Col. RS), M. A. S. Sobrino (Col. MAS), P. Bercedo y Lucía Arnáiz (Col. PBLA) y J. L. Zapata (Col. JLZ).

Los muestreos de campo realizados durante los últimos ocho años se efectuaron de manera no sistemática, dirigidos hacia la localización del mayor número posible de especies y por lo tanto centrados en hábitats potencialmente favorables para la presencia de cada una de las especies ya conocidas en Madrid, o citadas en provincias próximas de Castilla-La Mancha y Castilla-León. Un segundo grupo de campañas se dirigieron a la revisión de localidades con presencia histórica constatada de algunas especies, para determinar las posibilidades de permanencia de esas poblaciones. Finalmente otro grupo de muestreos se dirigieron a las zonas peor conocidas faunísticamente de Madrid, en las comarcas del sureste, en especial la Vega del Tajuña; estas campañas en el sureste han posibilitado la localización en Madrid de dos especies que no habían sido encontradas previamente en la región.

Cada registro, bibliográfico o no, fue asignado a una cuadrícula UTM de 10 x $10 \mathrm{~km}$. Los registros que podrían corresponder a más de una cuadrícula, bien por tratarse de términos amplios (por ejemplo «Orillas del Río Jarama»), o por coincidir en la confluencia de varias cuadrículas (por ejemplo «Cercedilla») fueron asignados arbitrariamente a una única cuadrícula. En los mapas de distribución no se han incluido puntos correspondientes a localidades ubicadas en provincias limítrofes ni aún cuando la cuadrícula correspondiente incluye parcialmente territorio de Madrid. La lista completa de registros y cuadrículas se presentan en los apéndices 1 (citas bibliográficas), 2 (observaciones de campo; cuando el número de ejemplares indicado es superior a cinco se trata de observaciones sin captura) y 3 (datos procedentes de colecciones). En los mapas (Figs. 1-7) se indican con diferente entramado las citas bibliográficas y de colecciones correspondientes a ejemplares colectados con anterioridad a 1980 (en gris) y las citas correspondientes a capturas efectuadas durante los últimos 25 años (en negro).

\section{Resultados y discusión}

El material examinado o citado en la bibliografía, procedente de la Comunidad de Madrid (Apéndices, 1, 2 y 3) corresponde a las especies siguientes:

\section{Cerocoma (Cerocoma) schaefferi (Linnaeus, 1758)}

Especie pan-europea de amplia distribución peninsular (Capote \& García-París, 2001) citada de forma general en la provincia de Madrid por Graells (1853) y en diversas localidades por Górriz Muñoz (1882), Rodríguez López-Neyra (1914, bajo el nombre de $C$. vahlii), Capote \& GarcíaParís (2001) y Pérez-Moreno et al. (2003).

Aparentemente dispersa por toda la región (Fig. 1), desde $480 \mathrm{~m}$ de altitud en Aranjuez, hasta los $1830 \mathrm{~m}$ del Puerto de los Cotos. Ocupa zonas abiertas o de matorral, próximas o no a masas forestales, tanto en sustratos silíceos como calcáreos o de yesos.

Las capturas recientes y observaciones de ejemplares adultos se efectuaron entre el 13 de mayo y el 9 de julio. Los adultos en general se localizaron sobre flores, sobre todo Helichrysum, Alchemilla, Santolina y Daucus.

Las localidades en las que no se han efectuado observaciones recientes se distribuyen por toda la región, sin que se concentren en áreas urbanas o periurbanas, por lo que parece que la ausencia de datos recientes en estos enclaves pueda explicarse por defectos de muestreo. En cualquier caso la especie parece mantener colonias saludables en numerosos puntos de la Comunidad y por lo tanto una comparación subjetiva entre datos antiguos y recientes parace sugerir que de momento la tendencia de la especie puede considerarse estable.

\section{Cerocoma (Metacerocoma) schreberi Fabricius, 1781}

Especie mediterránea distribuida por gran parte de la Península Ibérica citada en diversas localidades de Madrid por Martínez Sáez (1883), Capote \& García-París (2001) y Pérez-Moreno et al. (2003).

Se extiende por toda la región (Fig. 1), desde los $560 \mathrm{~m}$ de altitud en San Martín de la Vega, hasta los $1200 \mathrm{~m}$ en Cercedilla. Ocupa zonas abiertas o de matorral, próximas o no a masas forestales, tanto en sustratos silíceos como calcáreos o de yesos.

Las capturas recientes de ejemplares adultos se efectuaron entre el 12 de junio y el 15 de agosto. 
Los adultos se localizaron principalmente sobre flores, sobre todo Helichrysum, Retama y Santolina, con observaciones ocasionales en Eryngium, Daucus y Echium. En el sureste de Madrid a menudo se encuentra en compañía de $C$. schaefferi, aunque en general, cuando están juntas $C$. schreberi presenta densidades mucho más altas.

Las localidades en las que no se han efectuado observaciones recientes parecen concentrarse en áreas periurbanas del sur de la ciudad de Madrid. Pero la especie parece mantener colonias saludables en numerosos puntos de la Comunidad y por lo tanto una comparación subjetiva entre datos antiguos y recientes parace sugerir que de momento la tendencia de la especie puede considerarse estable o cuando menos no preocupante.

\section{Berberomeloe majalis (Linnaeus, 1758)}

Especie ibero-magrebí, abundante en gran parte de la Península Ibérica (García-París et al., 2003), muy frecuente en casi todo el territorio de Madrid (Graells, 1853). Las citas publicadas en la Comunidad de Madrid (a veces con los nombres de Meloe majalis var. fissicornis, M. m. var. laevigatus у M. m. var. maculifrons), corresponden a Górriz Muñoz (1882), Traizet (1895, 1896), Flach (1907), Rodríguez López-Neyra (1914), Sumakov (1934), Bologna (1989), García-París (1998), García-París et al. (2003) y Pérez-Moreno et al. (2003).

Se extiende por toda la región excepto en las zonas de alta montaña (Fig. 1), desde los $580 \mathrm{~m}$ de altitud en Villamanrique de Tajo, hasta los $1440 \mathrm{~m}$ del Puerto de Somosierra. Ocupa zonas abiertas o de matorral, próximas o no a masas forestales, tanto en sustratos silíceos como calcáreos o de yesos. Es la especie de meloido no volador más frecuente en las áreas de cultivos cerealistas de secano.

Las capturas recientes se efectuaron entre el 12 de marzo y el 23 de junio. Los adultos en general se localizaron caminando por suelo y se ha observado una alta mortalidad causada por atropello en carreteras, sobre todo en zonas de pastizales y trigales de altitud media.

Muchas de las localidades históricas de la especie (Aluche, Hortaleza, Canal), y especialmente de una de las formas de coloración totalmente negra (Vicálvaro, Santa Eugenia, Rivas de Jarama), han desaparecido bajo la urbanización y el ajardinamiento. Sin embargo la especie aún puede considerarse frecuente, especialmente en el sureste de la región, por lo que de momento consideramos que su estado de conservación todavía no es preocupante, excepto en las áreas periurbanas, donde antaño era una especie abundantísima.

\section{Lytta (Lytta) vesicatoria (Linnaeus, 1758)}

Especie pan-europea, frecuente en la mitad septentrional de la Península Ibérica (García-París et al., 2003) y en el norte de Madrid (Graells, 1853; De la Fuente, 1933 sub L. v. var. aurantiaca Escherich, 1894). Las citas precisas publicadas corresponden a Díaz Lizana (1864), Górriz Muñoz (1882) y García-París et al. (2003).

Se ha localizado a lo largo de la Sierra de Guadarrama y sus estribaciones, con penetraciones hasta el norte de la ciudad de Madrid (Fig. 1), tanto en zonas de altitud media como San Martín de Valdeiglesias a $760 \mathrm{~m}$ o Robledo de Chavela y Rozas de Puerto Real, a 850 m, como en zonas de alta montaña, hasta los $2100 \mathrm{~m}$ de altitud en las proximidades de la Laguna de los Pájaros. Ocupa zonas abiertas o de matorral, próximas en general a masas forestales, incluidos claros de bosque y bordes de caminos entre pinares, melojares y encinares, sobre sustratos silíceos, desde los piornales del piso oromediterráneo, a los retamares del límitesuperior del mesomediterráneo. Existen citas aisladas en el norte de la ciudad de Madrid (Hortaleza) que posiblemente correspondan a observaciones accidentales, ya que la especie es buena voladora.

Las observaciones de adultos se efectuaron entre el 28 de mayo y el 9 de julio. Todos los ejemplares adultos localizados se observaron sobre piornos y escobas (Cytisus) comiendo sus flores, involucrados en cortejo y cópula, o en reposo. En muchas ocasiones se ha observado en compañía de Mylabris hieracii.

Los datos parecen indicar que la especie presenta una tendencia estable, con poblaciones densas en el piso medio y alto de la Sierra de Guadarrama.

Fig. 1.- Mapas de distribución de especies de Cerocomini y Lyttini de la Comunidad de Madrid. En azul se señalan los registros anteriores a 1980 .

Fig. 1.- Distribution maps for the species of the tribes Cerocomini and Lyttini in Comunidad de Madrid. Presence of the species recorded before 1980 is indicated with blue dots. 

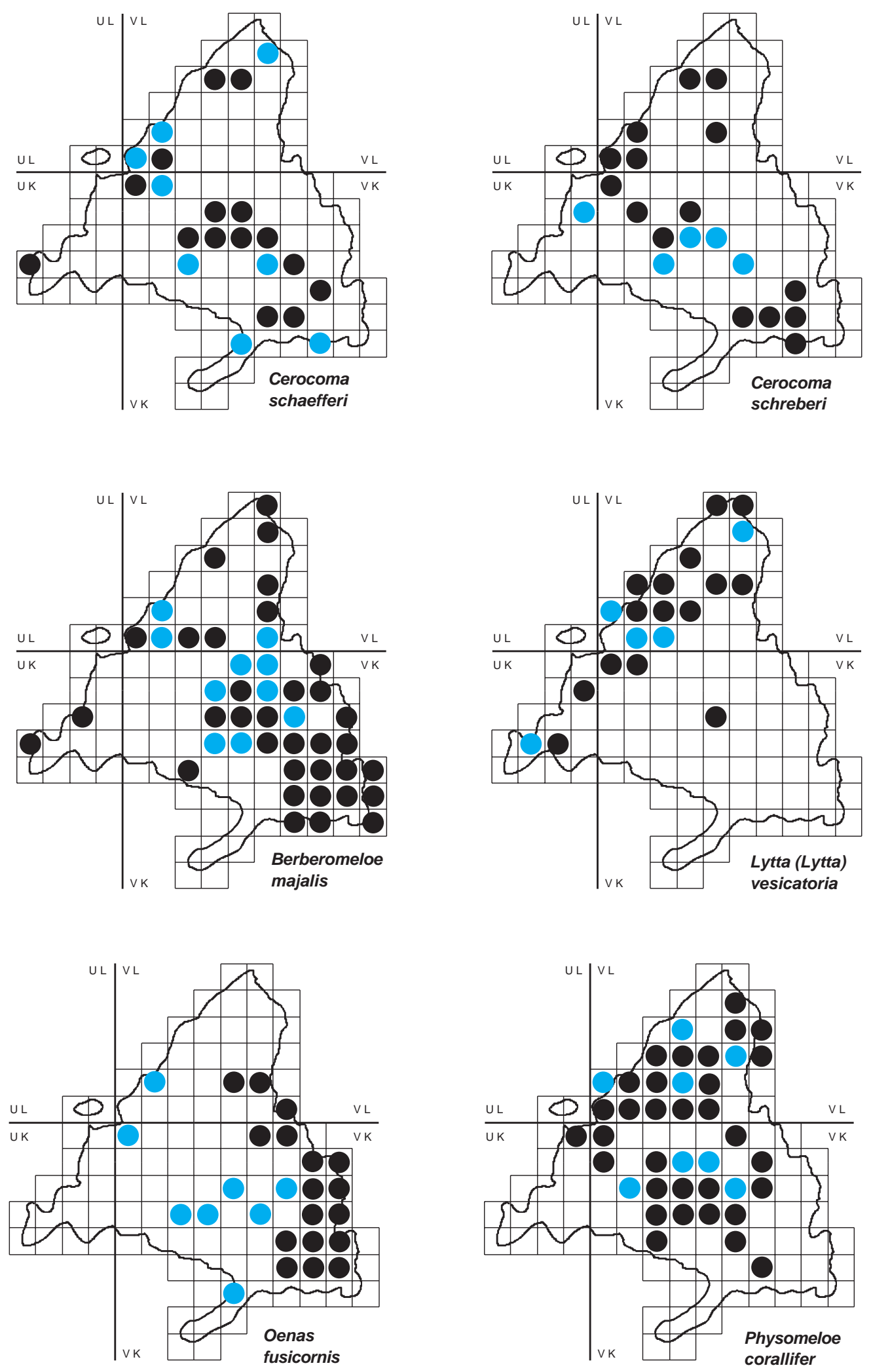
Oenas fusicornis Abeille de Perrin, 1880

Especie endémica de la Península Ibérica (García-París et al., 2003), distribuida por la mayor parte de la región de Madrid. Las citas publicadas corresponden a Rodríguez López-Neyra (1914, sub O. afer) y García-París et al. (2003).

Se ha localizado en la mayor parte de la región (Fig. 1), tanto sobre sustratos silíceos como calcáreos, desde los $480 \mathrm{~m}$ de altitud en Aranjuez, hasta los $1500 \mathrm{~m}$ de altitud del Ventorrillo (Cercedilla). Ocupa zonas abiertas o de matorral, próximas o no, a masas forestales sobre todo melojares, quejigares y encinares, aunque resulta una especie especialmente frecuente en los quejigares y en los yesos del sureste.

Las observaciones recientes de adultos se efectuaron entre el 28 de mayo y el 8 de julio. Los adultos se han observado comiendo principalmente flores de Ferula, Thapsia y Daucus. El cortejo y la cópula tienen lugar sobre las inflorescencencias de estas plantas. En muchas ocasiones las concentraciones de ejemplares sobre una misma inflorescencia son altísimas, de hasta más de 60 ejemplares. Se ha observado en compañía de Mylabris maculosopunctata, Hycleus scutellatus, Euzonitis quadrimaculata y ocasionalmente de M. quadripunctata. $\mathrm{Su}$ aparición en las flores coincide con el final de los picos de abundancia de Heliotaurus (Tenebrionidae: Alleculinae) con los que comparte una coloración similar y con los que posiblemente estén involucrados en complejos miméticos.

Todos los datos de distribución en los últimos 25 años se concentran en áreas del este, desde las calizas de Torrelaguna hasta los yesos de Alameda de las Fuentes, donde la especie mantiene poblaciones densas y abundantes. La falta de datos recientes en áreas occidentales parece sugerir un enrarecimiento de la especie en zonas subóptimas (áreas silíceas y zonas periurbanas) por lo que se recomienda la realización de muestreos específicos en dichas zonas.

\section{Physomeloe corallifer (Germar, 1818)}

Especie endémica de la Península Ibérica (García-París et al., 2003), distribuida por la mayor parte de la región de Madrid. Las citas publicadas corresponden a Graells (1853), Dieck (1870), Sanz de Diego (1880), Górriz Muñoz (1882), Traizet (1896), Flach (1907, sub Meloe c. var. evae), Sumakov (1934), Torres Sala (1962), Ortuño \& Hernández (1993), Bologna \& Aloisi (1994),
García-París et al. (2003) y Pérez-Moreno et al. (2003).

Se extiende por toda la región (Fig. 1), desde los $580 \mathrm{~m}$ de altitud de Gózquez de Abajo y los $600 \mathrm{~m}$ de Batres, hasta los $1790 \mathrm{~m}$ en el Puerto de la Fuenfría. Ocupa zonas abiertas o de matorral, próximas o no a masas forestales, predominantemente sobre sustratos silíceos, aunque también se ha encontrado en algunas localidades con yesos.

Las capturas recientes de ejemplares adultos se efectuaron entre el 16 de febrero y el 24 de mayo. Los adultos se observan cruzando los caminos o comiendo plantas herbáceas en pastizales abiertos.

Se trataba sin duda de la especie más abundante en los solares del centro, norte y oeste de la ciudad de Madrid, donde ocupaba descampados, a veces de escasas dimensiones. Todas las poblaciones urbanas de las que teníamos constancia han desaparecido, posiblemente como consecuencia de la desaparición de los Apoidea a los que atacan, al transformarse estos lugares en zonas ajardinadas sin la cohorte de plantas ruderales que permitían la existencia de las colonias de abejas. En el resto de la Comunidad la especie puede considerarse frecuente, aunque de continuar el uso de herbicidas en áreas periurbanas y carreteras, acompañado de una urbanización creciente, posiblemente tenga que cambiar de estatus en un futuro próximo.

\section{Meloe (Eurymeloe) baudueri Grenier, 1863}

Especie citada en la ciudad de Madrid por Pérez-Moreno et al. (2003) sin que exista ninguna otra documentación de su presencia en la región (Fig. 2).

\section{Meloe (Eurymeloe) brevicollis Panzer, 1793}

Especie citada de forma general en la provincia de Madrid por De la Fuente (1933) y en Somosierra por Pérez-Moreno et al. (2003).

Se trata de una especie bien distribuida por la Sierra de Guadarrama y sus aledaños, con citas procedentes de las colecciones históricas y también con citas recientes en la zona (Fig. 2), que se extienden desde los $640 \mathrm{~m}$ de altitud en Villaviciosa de Odón, hasta 1780 m en el Puerto de Navafría. Los ejemplares observados se localizaron en pastizales de montaña o cruzando la carretera en zonas de pinar, entre el 23 de abril y el 8 de mayo, aunque existe un registro antiguo en el mes de octubre y otro en junio. 
La especie parece mantener su presencia en zonas de montaña, pero en los últimos 25 años no ha vuelto a registrarse en zonas silíceas de cota baja, atestiguadas por las citas históricas de Villaviciosa y Madrid. La aparente desaparición de estas poblaciones y la escasez de los registros recientes indican la necesidad de efectuar muestreos específicos para determinar densidades de población y su estado de conservación. La tendencia aparente es de retroceso poblacional.

\section{Meloe (Eurymeloe) mediterraneus G. Müller, 1925}

Especie citada en la ciudad de Madrid por Rodríguez López-Neyra (1914, sub M. rugosus) y por Pérez-Moreno et al. (2003).

Las poblaciones de la especie se distribuyen por gran parte del área silícea occidental y septentrional de la región (Fig. 2), desde los $620 \mathrm{~m}$ de altitud en Brunete hasta los $1120 \mathrm{~m}$ en Garganta de Los Montes. Los ejemplares de la Comunidad de Madrid se han observado generalmente bajo piedras, en zonas de encinar-melojar, con registros en los meses de octubre, noviembre y mayo. Su presencia en parques urbanos de Madrid ha sido constatada hasta hace menos de una década (Dehesa de la Villa).

Como en el caso de $M$. proscarabaeus, muchas de las citas históricas se localizan en áreas de poca altitud (Brunete, Móstoles, Campamento), mientras que los registros recientes se localizan sobre todo en la Sierra de Guadarrama y sus aledaños (Garganta de los Montes, Colmenar Viejo). Es muy posible que esta especie se encuentre en retroceso en las zonas de menor altitud, aunque la escasez general de citas no permite evaluarlo.

Meloe (Eurymeloe) murinus Brandt et Erichson, 1832

Especie citada en la provincia de Madrid por Górriz Muñoz (1882) y De la Fuente (1933) y en Madrid por Rodríguez López-Neyra (1914). En las colecciones históricas existen ejemplares de la ciudad de Madrid y de Alcalá de Henares, mientras que las capturas recientes se centran en pastizales en dehesas con encinas dispersas en Colmenar Viejo y sus alrededores, a finales de octubre y principios de noviembre (Fig. 2). Los registros se localizan entre los $580 \mathrm{~m}$ y los $960 \mathrm{~m}$ de altitud.

\section{Meloe (Eurymeloe) nanus Lucas, 1849}

Especie no citada previamente en Madrid, pero representada en las colecciones históricas del
MNCN (Pérez Arcas) con ejemplares de Aranjuez y Montarco (Rivas de Jarama) y vuelta a localizar recientemente en los alrededores de Tielmes (Fig. 2 ). Todas las observaciones se han efectuado en los cerros yesíferos del sureste, entre $480 \mathrm{~m}$ y $680 \mathrm{~m}$ de altitud, las actuales, bajo piedras en pequeños pastizales entre atochares de ladera en noviembre. La localidad de Montarco corresponde a una antigua estación de tren (del conocido tren de Arganda), que sin duda puede considerarse como uno de los lugares históricos mejor muestreados entomológicamente de la Comunidad de Madrid, de donde se han descrito numerosos taxones, y que hoy ha desaparecido completamente bajo las urbanizaciones.

\section{Meloe (Eurymeloe) rugosus Marsham, 1802}

Especie citada en Madrid por Rodríguez LópezNeyra (1914) y en la provincia por De la Fuente (1933). García-París \& Ruiz (2005) consideran que estas citas corresponden a M. mediterraneus, y en el caso de Madrid, esta afirmación se confirma con el examen del material del MNCN que utilizó Rodríguez López-Neyra para indicar su presencia en Madrid. Las observaciones de esta especie en Madrid se limitan por lo tanto a dos ejemplares encontrados tras las lluvias otoñales, en octubre, en un prado entre encinas cercano al jaral, en terrenos pizarrosos de los alrededores del Atazar (Fig. 2). La frecuencia de observación de esta especie es por lo tanto muy baja, pero a la vista de los reultados de los muestreos de los últimos años, no difiere realmente de la de la mayoría de las especies del género, que en general se limitan a unas pocas capturas.

\section{Meloe (Coelomeloe) tuccius (Rossi, 1792)}

Especie de amplia distribución mediterránea. Citada de forma general en la provincia de Madrid por Graells (1853). Las citas precisas publicadas de Madrid corresponden a Górriz Muñoz (1882), Traizet (1896), Sumakov (1934), Torres Sala (1962) y Pérez-Moreno et al. (2003).

Las observaciones de esta especie se han realizado entre el 17 de marzo y el 28 de abril, con un registro en mayo; entre $710 \mathrm{~m}$ y $900 \mathrm{~m}$ de altitud. En la bibliografía ibérica esta especie se considera relativamente común y esta idea se ha visto reforzada en Madrid, porque de hecho, muchas de las observaciones de esta especie se han efectuado en parques y solares de barrios de la ciudad Madrid (Alameda de Osuna, Aluche, Campamento, Casa de Campo, 

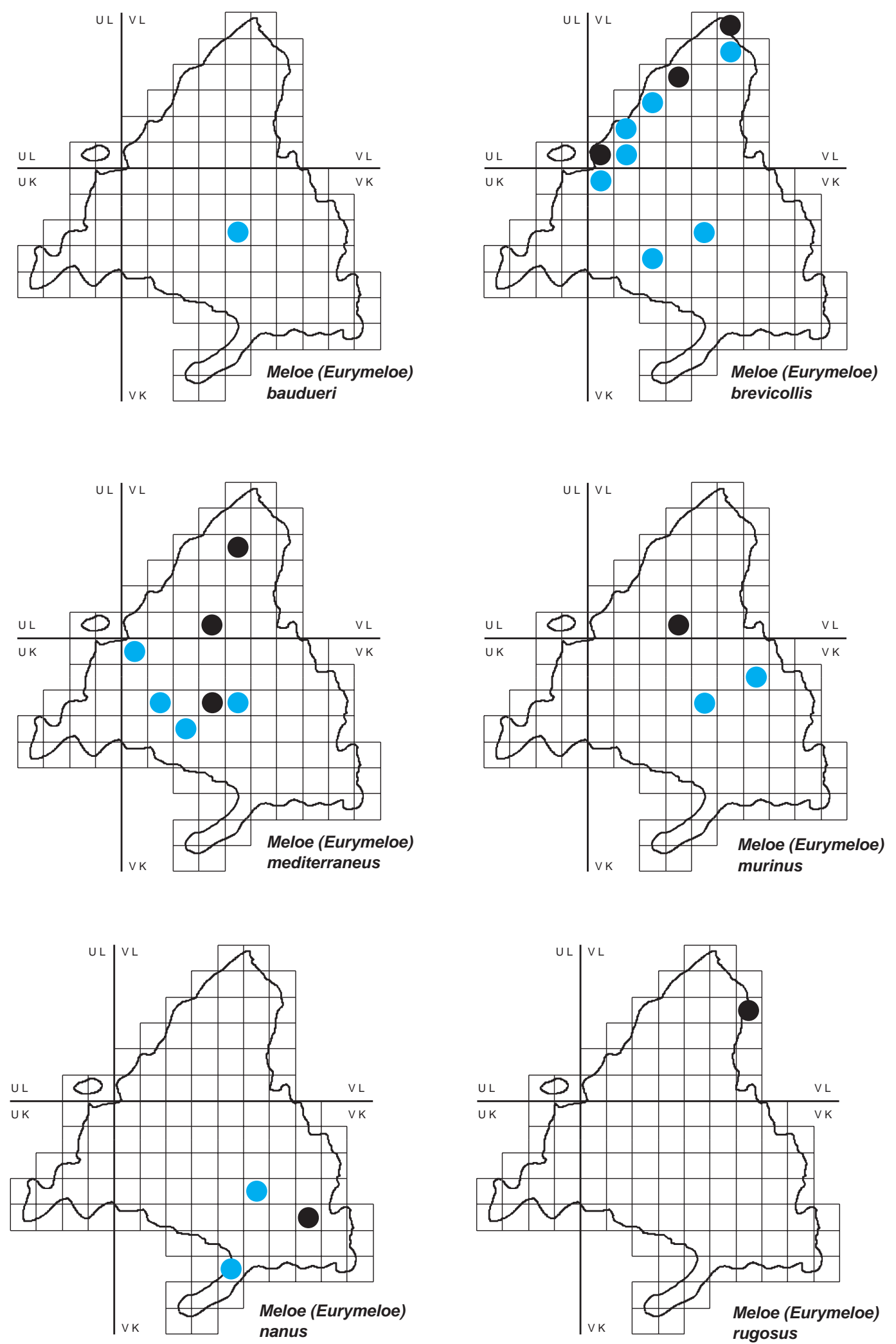
Chamartín, Ciudad Universitaria, Dehesa de la Villa, Puerta de Hierro, Vicálvaro, Vallecas, Virgen del Cortijo...). Pero al examinar el listado completo de registros y de ejemplares de colección resulta llamativo, que excepto contadas excepciones (El Atazar, Paracuellos y Torres de la Alameda), todos los ejemplares se han colectado, precisamente en áreas periurbanas de Madrid (Fig. 3). El disparatado crecimiento urbano de los últimos 10 años de Madrid y de todas las ciudades periféricas ha ocasionado la desaparición de los solares y descampados donde vivía la especie (casos constatados en Aluche, Campamento, Vicálvaro y Virgen del Cortijo). En otros casos ese crecimiento urbano ha provocado el aislamiento de los descampados y de los parques, que a su vez han sufrido una importante labor "restaudora" que ha acabado con casi cualquier vestigio de vegetación autóctona en los mismos (Dehesa de la Villa, Ciudad Universitaria). Como consecuencia, esta especie tradicionalmente considerada "vulgar", bien representada hace 20 años en parques y solares, únicamente parece mantener colonias estables en poquísimas localidades de la Comunidad de Madrid. Se trata por lo tanto de una especie en franca regresión que posiblemente requiera la toma de medidas para garantizar su conservación.

\section{Meloe (Lampromeloe) cavensis Petagna, 1819}

Especie citada en la provincia de Madrid sin más precisión por Graells (1853, como Meloe aeneus Laporte de Castelnau, 1840) y por De la Fuente (1933). Se encuentra bien representada en las colecciones históricas de Madrid, a partir de cuyos datos podemos indicar que a finales del siglo XIX y principios del siglo XX era una especie relativamente común en las afueras del sur de la ciudad (Madrid y Vallecas) y que estaba bien distribuida por los terrenos yesíferos del sur (Aranjuez) (Fig. 3). El último ejemplar localizado en Madrid del que tenemos constancia, debió de ser capturado hacia 1910. Dado su gran tamaño, su llamativo colorido verde metálico y púrpura, y su actividad a pleno sol en los meses más fríos del año, no se trata de una especie que pase desapercibida y por lo tanto la falta de registros (tanto en las colecciones, como en los muestreos específicos centrados durante más de cinco años en las localidades de donde ha sido citada) sugiere una desaparición real. Posiblemente pueda ser considerada como una especie ya extinguida en Madrid.

\section{Meloe (Lampromeloe) variegatus Donovan, 1793}

Esta especie no ha sido citada previamente en Madrid, pero como en el caso de la especie anterior, existen tres ejemplares capturados en diversas localidades de la geografía madrileña (Perales de Tajuña, Rozas de Puerto Real y Madrid), todos ellos anteriores a 1904. La distribución de las localidades permite aventurar que la especie debió de estar bien repartida por todo el sur de la región, desde los terrenos silíceos del suroeste a los calcáreos del sureste (Fig. 3). Su presencia en zonas relativamente bien conservadas y poco muestreadas (encinares y melojares del suroeste y quejigares del sureste) no permiten descartar la presencia actual de $M$. variegatus en Madrid, aunque como en el caso de la especie anterior, si aún existe, debería considerarse en la categoría de En Peligro de Extinción.

\section{Meloe (Meloe) proscarabaeus Linnaeus, 1758}

Especie citada de forma general en la provincia de Madrid por Górriz Muñoz (1882) y De la Fuente (1933) y de forma concreta en varias localidades por Pérez-Moreno et al. (2003). Además de los datos bibliográficos se ha revisado material relativamente abundante, teniendo en cuenta la escasez general del género Meloe en Madrid, de varios puntos de la geografía madrileña. Los datos de las colecciones históricas extienden la distribución de la especie, desde las laderas silíceas de la Sierra de Guadarrama, hasta las vegas sobre terrenos calcáreos del sureste (Perales de Tajuña, Villarejo de Salvanés), pasando por los yesares del centro - sur (Rivas, Vallecas, Paracuellos del Jarama, Pinto) (Fig. 3). Sin embargo, a pesar de los muestreos específicos centrados en el sureste de la Comunidad, todos los registros y observaciones recientes se han efectuado exclusiva-

Fig. 2.- Mapas de distribución de las especies del subgénero Eurymeloe (Meloini) de la Comunidad de Madrid. Los registros anteriores a 1980 se señalan en color azul.

Fig. 2.- Distribution maps of the species of the subgenus Eurymeloe (Meloini) from Comunidad de Madrid. Presence of the species recorded before 1980 is indicated with blue dots. 
mente en la Sierra de Guadarrama y sus aledaños, por lo que la especie puede considerarse en retroceso poblacional.

\section{Meloe (Meloe) violaceus Marsham, 1802}

Especie citada en Madrid por Pérez-Moreno et al. (2003), ampliamente distribuida en la Sierra del Guadarrama a finales del siglo XIX y primera mitad del siglo XX, según atestiguan las colecciones históricas del MNCN, pero que no cuenta con ningún registro ni observación realizada en los últimos 25 años como mínimo (Fig. 3). La presencia actual de esta especie no es descartable, ya que por tratarse de una especie de montaña podría mantener poblaciones acantonadas en áreas de relieve favorable. En cualquier caso la falta de registros recientes de esta especie llamativa es una clara indicación de un marcado retroceso poblacional.

\section{Meloe (Treiodus) autumnalis Olivier, 1792}

Especie citada en Madrid por Graells (1853, como Meloe cyaneus Fabricius, 1801). Se trata de una especie poco frecuente, localizada tanto en áreas silíceas como calcáreas, pero que apenas cuenta con registros en Madrid y casi todos ellos localizados en la Sierra (Fig. 3). Además de los ejemplares señalados en los apéndices, se ha examinado un ejemplar de Villanueva de la Torre (Guadalajara, VK79) localizado a muy poca distancia del límite regional el 4XI-2004 (I. Martínez-Solano). No existen datos suficientes para evaluar su estado actual de conservación, aunque la distribución de citas claramente sugiere un retroceso poblacional.

Actenodia billbergi (Gyllenhal, 1817)

Especie endémica de la Península Ibérica y el sur de Francia (Bologna, 1991), citada en la provincia de Madrid por Graells (1853) y con citas concretas publicadas por Rodríguez López-Neyra (1914), Sumakov (1930, sub Hycleus bilbergi) y Pérez-Moreno et al. (2003).
Se extiende por toda la región excepto en las áreas de montaña (Fig. 4), desde los $560 \mathrm{~m}$ de altitud de Ciempozuelos, hasta los $1000 \mathrm{~m}$ en El Escorial. Ocupa zonas abiertas o de matorral, próximas o no a masas forestales, sobre sustratos silíceos, calizos o de yesos.

Las capturas recientes de ejemplares adultos se efectuaron entre el 18 de mayo y el 23 de junio. Los adultos se ecuentran sobre flores diversas.

Se trata de una especie relativamente frecuente, aunque con densidades bajas.

\section{Hycleus dufourii (Graells, 1849)}

Especie endémica de la Península Ibérica, descrita de Guadarrama por Graells (1849). Las citas publicadas de Madrid corresponden a Graells (1849, 1851a, 1851b, 1853), Górriz Muñoz (1882), Marseul (1870), De la Fuente (1933), Trotta-Moreu \& García-París (2001) y Pérez-Moreno et al. (2003).

En Madrid se localiza únicamente en la Sierra, desde los aldedores de El Escorial hasta Montejo de la Sierra (Fig. 4). Sus registros se distribuyen desde los 1000 m de altitud en El Escorial, hasta casi los $1900 \mathrm{~m}$ del Collado de Marichiva. Ocupa zonas abiertas o de matorral, próximas a pinares y melojares, sobre sustratos silíceos.

Las capturas recientes de ejemplares adultos se efectuaron entre el 14 y el 21 de julio. Los adultos se han encontrado en flores de Rubus y con menos frecuencia de Senecio.

Se trata de una especie muy poco frecuente, que presenta densidades bajas. Las capturas y observaciones efectuadas en los últimos 25 años se concentran en los alrededores del Escorial, y aunque un incremento del muestreo permita localizar otras poblaciones, en el estado actual debe considerarse como una especie en regresión.

\section{Hycleus duodecimpunctatus (Olivier, 1811)}

Especie endémica de la Península Ibérica y el sur de Francia (Bologna, 1991), citada de forma general en la provincia de Madrid (Graells, 1853 sub "Mylabris cyanescens F."). Las citas precisas publi-

Fig. 3.- Mapas de distribución de especies de Meloini de la Comunidad de Madrid. Los registros anteriores a 1980 se indican en color azul.

Fig. 3.- Distribution maps of the species of the tribe Meloini of Comunidad de Madrid. Blue dots represent localities where the species was present before 1980 without further observations. 

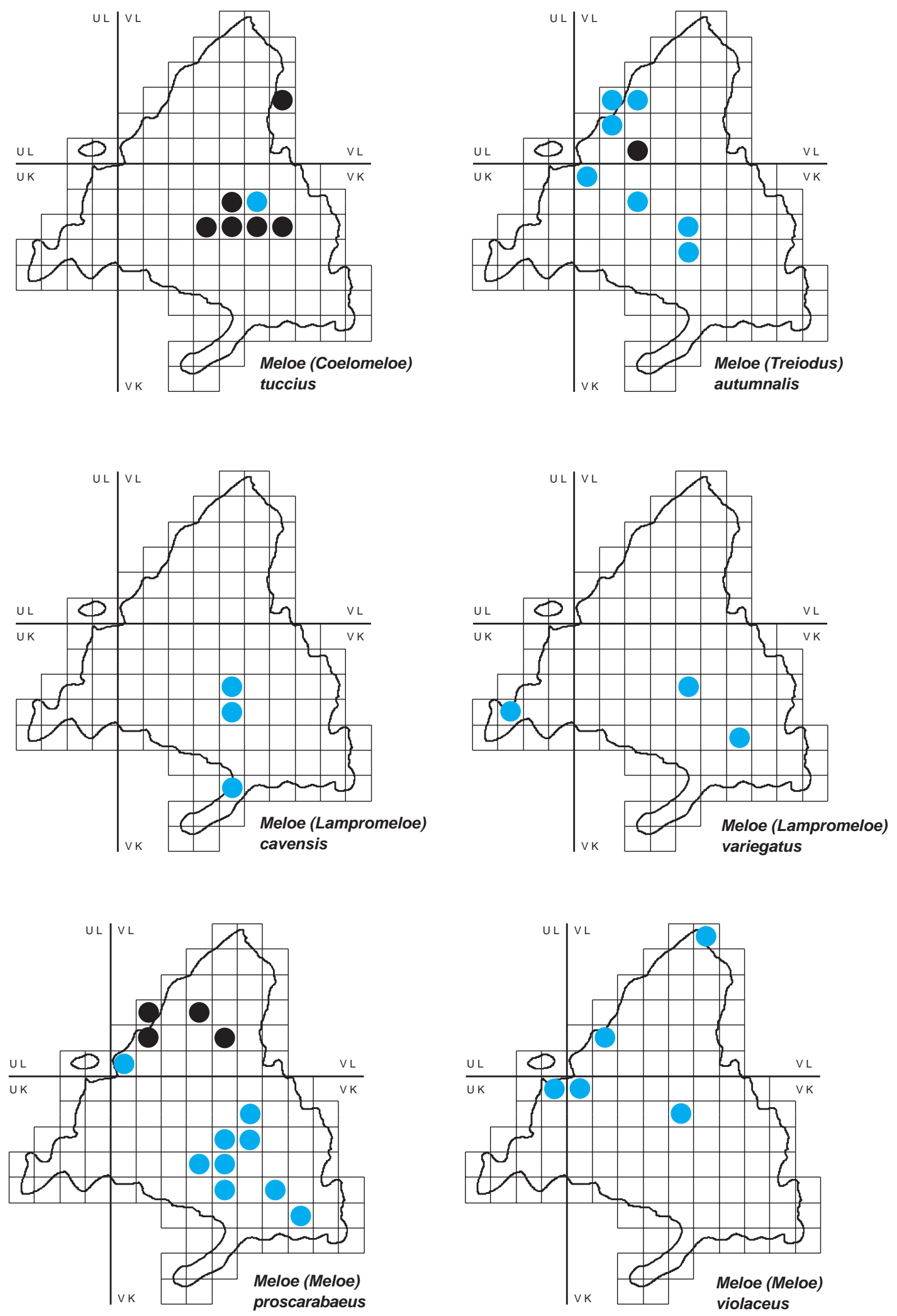
cadas de Madrid fueron recogidas por Górriz Muñoz (1882) y Trotta-Moreu \& García-París (2001).

La información disponible, basada sobre todo en datos históricos, indica que la especie se distribuye de forma dispersa por la mayor parte del territorio de la Comunidad de Madrid, excepto en tercio septentrional, donde la falta de citas posiblemente corresponda a defectos de muestreo (Fig. 4). Sus registros se distribuyen desde los los $480 \mathrm{~m}$ de altitud en Aranjuez, hasta los $1200 \mathrm{~m}$ de altitud en El Escorial y Navacerrada. Ocupa zonas abiertas o de matorral, indiferente con respecto al sustrato.

Las capturas recientes de ejemplares adultos se efectuaron entre el 23 de junio y el 23 de julio. La mayoría de los ejemplares adultos observados se encontraron en tallos secos de gramíneas o en flores de crucíferas.

Se trata de una especie poco frecuente, que puede presentar densidades muy altas a nivel local. Las capturas y observaciones efectuadas en los últimos 25 años son muy escasas con respecto al conjunto de datos anteriores, por lo que en ausencia de nuevos datos debe considerarse como una especie en regresión en Madrid.

\section{Hycleus scutellatus (Rosenhauer, 1856)}

Especie endémica de la Península Ibérica. Citada de forma general en la provincia de Madrid (Rodríguez López-Neyra, 1914; De la Fuente, 1933 como Zonabris decimpunctata), con citas concretas publicadas por Trotta-Moreu \& GarcíaParís (2001), Pérez-Moreno et al. (2003) y LópezColón (2005).

Bien distribuida por la mayor parte de Madrid excepto en el tercio norte donde faltan citas (Fig. 4). Sus registros se distribuyen desde los $460 \mathrm{~m}$ de altitud en Las Infantas, hasta los 1450 m en Santa María de la Alameda. Ocupa zonas abiertas o de matorral, con indiferencia del sustrato.

Las capturas recientes de ejemplares adultos se efectuaron entre el 15 de mayo y el 21 de julio. Los adultos se han encontrado en flores de Thapsia, Senecio y en flores de varias crucíferas de pequeño porte, aunque muchos registros corresponden a ejemplares localizados sobre tallos secos de gramíneas y otras plantas. Ocasionalmente forman agre- gaciones compactas que llegan a cubrir casi por completo las inflorescencias de las umbelíferas.

Se trata de una especie frecuente, abundante a nivel local, que presenta densidades puntuales muy altas. No se han localizado ejemplares en muchas de las áreas urbanas y periurbanas en donde se encontraba hace más de 25 años, pero el resto de poblaciones parece encontrarse en estado satisfactorio.

\section{Mylabris (Chalcabris) uhagonii Martínez Sáez, 1873}

Especie endémica de la Península Ibérica. Las citas precisas publicadas de Madrid corresponden a la descripción original de Martínez Sáez (1873) y a los trabajos de Górriz Muñoz (1882), Rodríguez López-Neyra (1914) y Sumakov (1930).

En las colecciones del Museo existen 118 ejemplares de esta especie capturados en un total de 11 localidades diferentes de la Comunidad de Madrid, distribuidos desde los yesos del sur y sureste hasta El Escorial, aunque la mayor parte de los registros se concentran en las zonas yesíferas localizadas dentro de y al sur del municipio de Madrid (Fig. 4), durante los meses de junio y julio. Todas estas capturas excepto una corresponden a fechas anteriores a 1920 (las fechas registradas se extienden desde 1906 a 1916). La única captura posterior a estas fechas corresponde a un ejemplar capturado en Getafe por S. V. Peris posiblemente en los años 70 ( $\sin$ datos de fecha en la etiqueta). Los muestreos específicos destinados a la localización de nuevos ejemplares en todas las localidades conocidas y en otras próximas, realizados en los últimos ocho años han sido completamente infructuosos.

A la vista de los datos, se trataba de una especie abundante, que incluso colonizaba los jardines y parques urbanos de la ciudad de Madrid (Salón del Prado, Casa de Campo), que ha desaparecido de la Comunidad de Madrid. La existencia de un registro de los años 70 no permite descartar su presencia actual y considerarla una especie extinguida en Madrid, por lo que ha de incluirse en la categoría de En Peligro de Extinción, y deben reforzarse los muestreos para tratar de localizar alguna población superviviente de este endemismo ibérico, único representante occidental del subgénero Chalcabris,

Fig. 4.- Mapas de distribución de especies de Mylabriini de la Comunidad de Madrid. En azul los registros anteriores a 1980.

Fig. 4.- Distribution maps of the species of Mylabrini of Comunidad de Madrid. Localities recorded before 1980 are indicated with blue dots. 

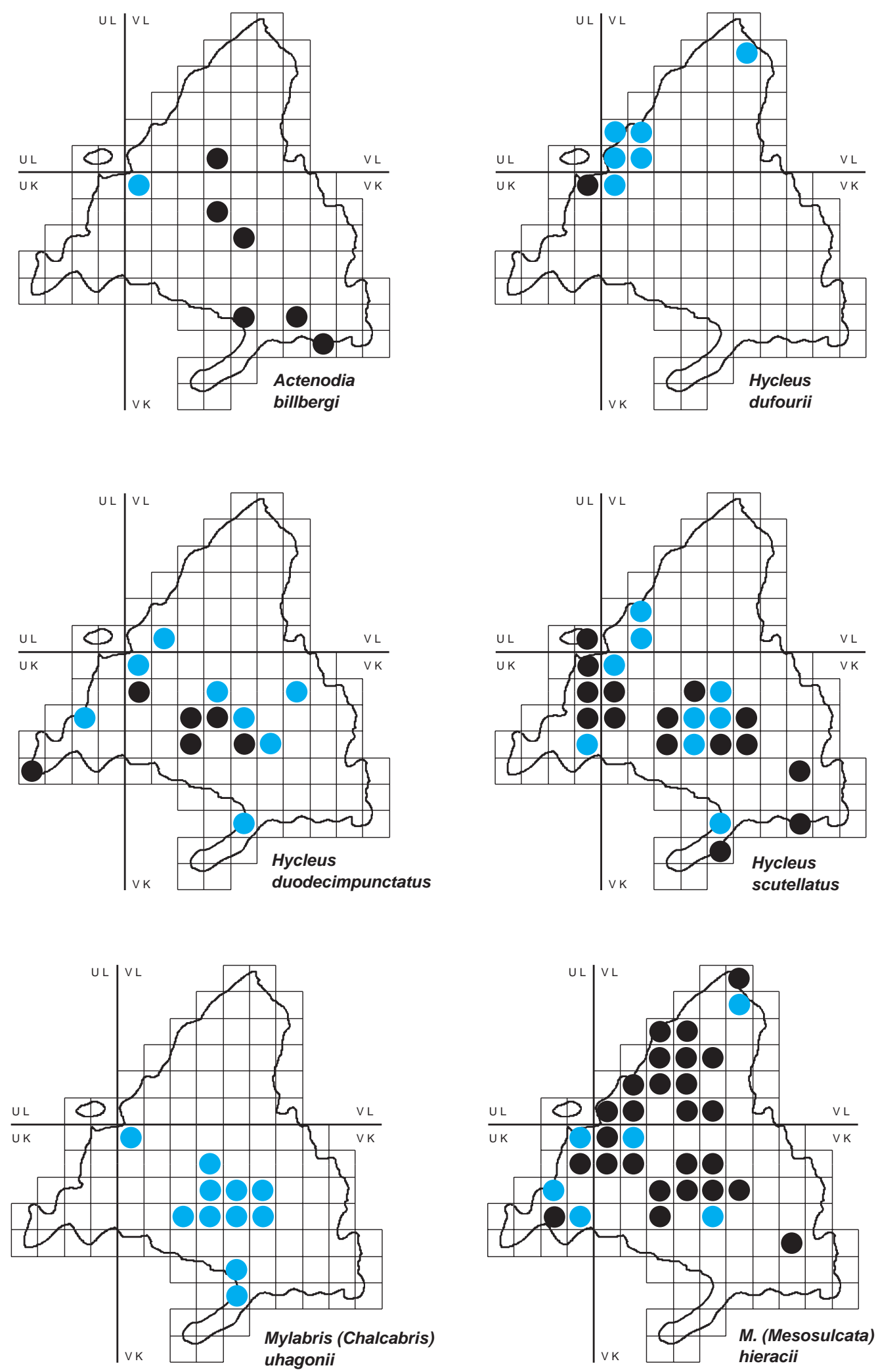
cuyas especies más próximas se localizan en el Caúcaso. Las causas de su desaparición posiblemente se encuentren en el posible retroceso de las poblaciones de la especie de ortóptero de cuyas ootecas se alimentarían sus larvas, aunque al desconocerse cúal es, no se pueden tomar las medidas oportunas.

\section{Mylabris (Mesosulcata) hieracii Graells, 1849}

Endemismo de la Península Ibérica, con un único registro en el suroeste de Francia (Bologna, 1991). Citado en Madrid por Graells (1849, 1851a, 1851b, 1853), Seidlitz (1867), Marseul (1870), Górriz Muñoz (1882), Fuente (1933, sub $M$. $h$. var. suspiciosa), Bologna (1991) y Pérez-Moreno et al. (2003).

Bien distribuido por las Sierras de Guadarrama y Ayllón y sus estribaciones, con citas dispersas en el resto de la Comunidad (Fig. 4). Sus registros se distribuyen desde los $590 \mathrm{~m}$ de altitud en los embalses del Alberche, hasta los $1850 \mathrm{~m}$ de los alrededores del Puerto de los Cotos. Ocupa sobre todo claros de bosque y pastizales en pinares, rebollares y encinares supramediterráneos sobre sustrato silíceo, aunque ocasionalmente se ha localizado en áreas abiertas sobre sustratos yesíferos.

Las capturas recientes de ejemplares adultos se efectuaron entre el 18 de mayo y el 7 de agosto, con registros aislados en marzo. Los adultos se han encontrado sobre todo en flores de Cytisus y Genista, aunque ocasionalmente, sobre todo en fechas tardías, se encuentran sobre flores de otras plantas. A menudo se concentran numerosos ejemplares en unos pocos pies de planta (Senecio...).

Se trata de una especie frecuente, abundante a nivel local, que presenta densidades puntuales altas. La distribución de los datos históricos se corresponde con la de los datos actuales excepto en algunos puntos meridionales.

\section{Mylabris (Micrabris) dejeani (Gyllenhal, 1817)}

Especie endémica de la Península Ibérica, citada en la Provincia de Madrid por Graells (1853) y De la Fuente (1933) y en diversas localidades madrileñas por Górriz Muñoz (1882) y PérezMoreno et al. (2003).

Esta especie se encuentra bien representada por citas históricas en áreas del centro de Madrid y de la Sierra de Guadarrama (Fig. 5), desde los $610 \mathrm{~m}$ de altitud de Montarco, hasta los $1700 \mathrm{~m}$ del Puerto de la Morcuera. Sin embargo los únicos registros relativamente recientes corresponden a los publicados por Pérez-Moreno et al. (2003) del Embalse de Navacerrada-Collado Mediano. Se trata por lo tanto de una especie en franca regresión que podría estar desapareciendo de gran parte del territorio de la Comunidad de Madrid, especialmente del centro y sur, donde se han concentrado los muestreos.

Mylabris (Micrabris) maculosopunctata Graells, 1858

Especie endémica de la Península Ibérica, citada en Madrid por Rodríguez López-Neyra (1914) y Pérez-Moreno et al. (2003).

Sus registros se encuentran dispersos por toda la Comunidad de Madrid, con datos antiguos y recientes que no muestran un patrón diferenciado (Fig. 5). Las citas se distribuyen desde los $480 \mathrm{~m}$ de altitud de Aranjuez, hasta los $1200 \mathrm{~m}$ de los alrededores del Escorial. Ocupa sobre todo zonas abiertas sobre cualquier tipo de sustrato.

Las capturas recientes de ejemplares adultos se efectuaron entre el 11 de junio y el 1 de julio. Las mayores concentraciones de adultos se han observado sobre flores de Thapsia y Echium, aunque se encuentran sobre flores de muchas otras plantas.

Se trata de una especie con fluctuaciones poblacionales muy marcadas, que puede presentar densidades altísimas a nivel local (como las observadas en Tielmes en el año 2000) para desaparecer casi por completo en años sucesivos (sin observaciones en el mismo punto en los años 2001, 2003, 2004 y 2005; y con sólo tres ejemplares observados en el 2002). No disponemos de datos suficientes para evaluar posibles tendencias.

\section{Mylabris (Micrabris) sobrina Graells, 1849}

Endémismo ibérico descrito de Madrid (Graells, 1849) y citado repetidamente por Graells (1851a, 1851b, 1853), Seidlitz (1867), Marseul (1870), Górriz Muñoz (1882), Sumakov (1930) y PérezMoreno et al. (2003).

En Madrid la especie está limitada a las Sierras de Guadarrama y Ayllón (Fig. 5), localizada desde los $1200 \mathrm{~m}$ de altitud en El Escorial, hasta los 2100 $\mathrm{m}$ de los alrededores de la Laguna de los Pájaros. Ocupa los piornales y pastizales culminícolas de los pisos oro y crioromediterraneo, y los claros de bosque del pinar y melojar supramediterráneo.

Las capturas recientes de ejemplares adultos se efectuaron entre el 8 de junio y el 7 de agosto. Los 
adultos se concentran en las flores de piornos (Cytisus) y con menor densidad en Rubus, Genista, Senecio y Linaria, aunque ocasionalmente se encuentran en flores de otras plantas. En muchos lugares se suele encontrar en compañía de Mylabris hieracii y Lytta vesicatoria.

Se trata de una especie frecuente, que puede presentar densidades muy altas a nivel local, y que parece mantener poblaciones saludables.

\section{Mylabris (Micrabris) varians (Gyllenhal, 1817)}

Endémismo ibérico citado de la provincia de Madrid por Graells (1853) y De la Fuente (1933, sub Zonabris varians var. tripunctatofasciata) y de localidades precisas por Pérez-Moreno et al. (2003).

En la Comunidad de Madrid parece presentar dos núcleos poblacionales disjuntos, uno localizado en la Sierra de Guadarrama-Ayllón y el otro en las áreas calizas del sureste (Fig. 5). Las poblaciones de la Sierra se localizan en pastizales oromediterráneos y en claros de bosques de pinos y rebollos supramediterráneos, entre los $1300 \mathrm{~m}$ de los alrededores de El Escorial y los $1800 \mathrm{~m}$ del Puerto de la Fuenfría (aunque supera los $1900 \mathrm{~m}$ en las faldas del cercano pico Montón de Trigo, ya en Segovia). Las poblaciones del este y sureste se localizan en las proximidades de melojares, pero también en terrenos abiertos sobre margas yesos y calizas, entre los $610 \mathrm{~m}$ de altitud de Montarco y los $850 \mathrm{~m}$ de Nuevo Baztán.

Las capturas recientes de ejemplares adultos se efectuaron entre el 20 de junio y el 21 de agosto. En zonas altas de la Sierra los adultos son frecuentes en flores de margaritas, cardos de bajo porte y otras pequeñas compuestas y con menor frecuencia en Linaria, mientras que en zonas de altitud media se localizan principalmente en flores de dipsacáceas (Knautia-Scabiosa). En el sureste suelen encontrarse en flores de compuestas y dipsacáceas, pero también de Daucus, Thapsia y Echium. En muchos lugares se localiza en compañía de Mylabris quadripunctata.

Se trata de una especie poco frecuente, aunque bien representada, que parece mantener poblaciones estables en su área de distribución conocida.

\section{Mylabris (Mylabris) amorii Graells, 1858}

Especie endémica de la Península Ibérica, citada en la provincia de Madrid por Sumakov (1930) y De la Fuente (1933), aunque sin precisar localidades.

En la colección del MNCN existe una serie de 135 ejemplares recolectados en mayo de 1909 en El Rincón, sin que la especie haya vuelto a ser localizada en ningún otro punto de Madrid (Fig. 5). Esta especie debería buscarse con intensidad tanto en el suroeste, de donde proceden los ejemplares citados, como en el sureste, ya que las condiciones ecológicas de los quejigares sobre calizas de Madrid son muy parecidas a las que presentan las poblaciones de esta especie en la provincia de Cuenca.

\section{Mylabris (Mylabris) quadripunctata (Linnaeus, 1767)}

Especie euroasiática (Bologna, 1991), citada en Madrid por Amor Mayor (1860), Górriz Muñoz (1882) y Pérez-Moreno et al. (2003) y a nivel provincial por De la Fuente (1933, sub Zonabris q. var. adamsi Fischer, 1824).

Sus registros se encuentran bien distribuidos por toda la Comunidad de Madrid, con datos antiguos y recientes que no muestran un patrón diferenciado, excepto en el área urbana y periurbana de la ciudad de Madrid, en donde faltan registros recientes (Fig. 5). Las citas se distribuyen desde los $480 \mathrm{~m}$ de altitud de Aranjuez, hasta los $1800 \mathrm{~m}$ de los alrededores del Puerto de la Fuenfría. Especie frecuente al borde de caminos y carreteras y en zonas abiertas, sobre cualquier tipo de sustrato.

Las capturas recientes de ejemplares adultos se efectuaron entre el 3 de junio y el 18 de agosto. Los adultos se han encontrado sobre tallos secos de gramíneas, y sobre flores de plantas muy diversas, Senecio, Thapsia, Daucus... y sobre todo de Scolymus. No suelen formar agregaciones tan compactas como las de otras especies.

Se trata de una especie con fluctuaciones poblacionales marcadas, aunque las diferencias interanuales observadas no son tan patentes como en el caso de $M$. maculosopunctata, quizás porque esta última especie presenta un periodo de actividad más reducido. Exceptuando las áreas urbanas, de donde parece haber desaparecido, el resto de poblaciones parece mantenerse relativamente estable, aunque en especies tan frecuentes como ésta no es posible evaluar cambios con fiabilidad a corto plazo.

Mylabris (Mylabris) variabilis (Pallas, 1781)

Especie euroasiática (Bologna, 1991), citada en la provincia de Madrid por Graells (1853) y en 

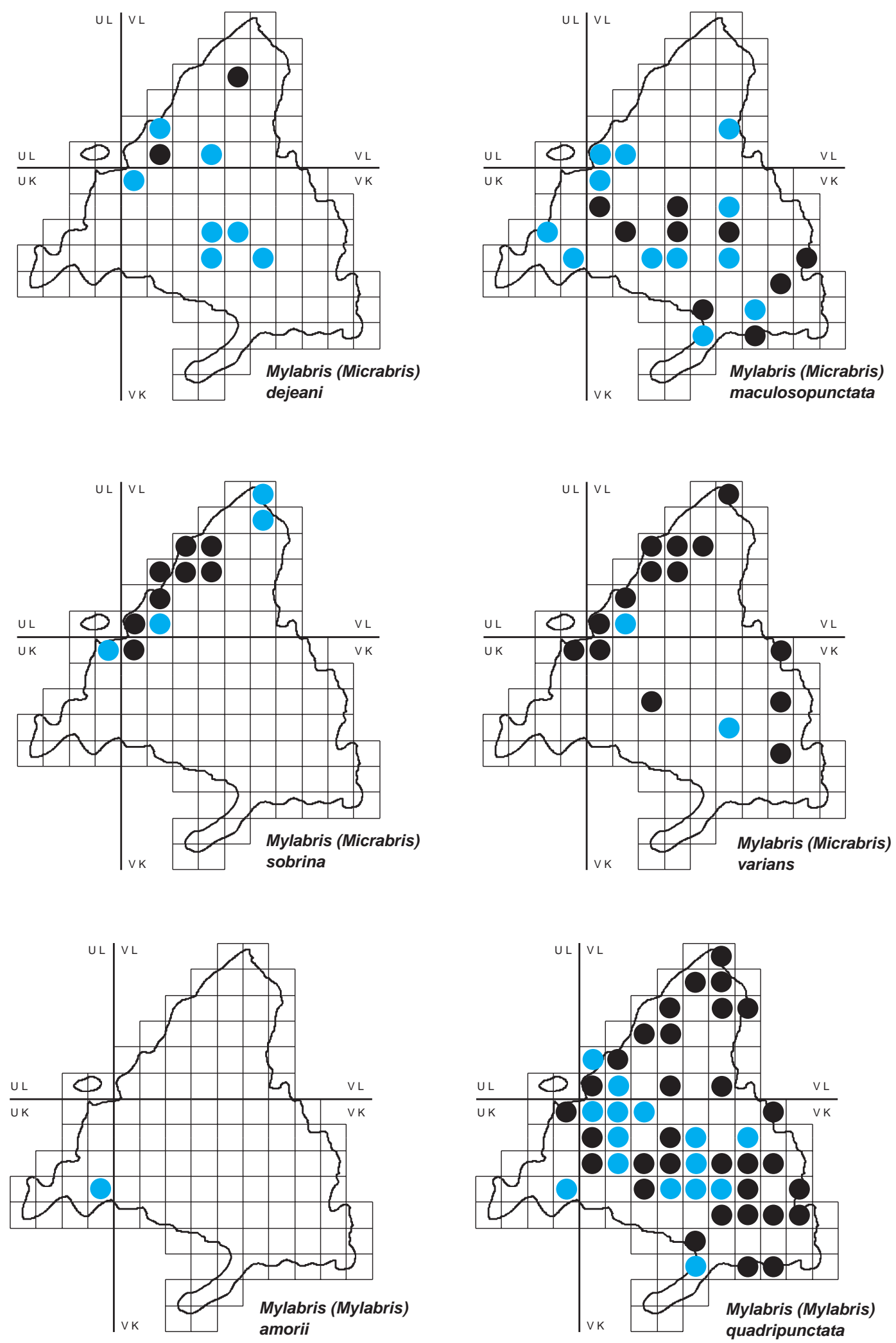
localidades concretas por Górriz Muñoz (1882), Gómez Carrasco (1888), Sumakov (1930) y PérezMoreno et al. (2003).

Parece encontrarse distribuida por la mayor parte de la Comunidad de Madrid, aunque los datos recientes son mucho menos numerosos que los antiguos, ya que de las 17 cuadrículas de ocupación, en los últimos 25 años sólo ha sido registrada en cinco (Fig. 6). Sus registros se distribuyen desde los $480 \mathrm{~m}$ de altitud de Aranjuez, hasta los $1510 \mathrm{~m}$ del Puerto de Guadarrama. Ocupa sobre todo zonas abiertas, próximas a campos de cultivo, o pastizales entre pinares, sobre cualquier tipo de sustrato.

Las capturas recientes de ejemplares adultos se efectuaron entre el 23 de junio y el 28 de julio. Los adultos se encuentran en flores diversas, generalmente en cardos de poco porte, y sobre Daucus, Scabiosa, Thapsia, Eryngium, Echium y Scolymus.

Se trata de una especie poco frecuente, aunque puede presentar altas densidades a nivel local. La comparación de los datos antiguos y recientes apunta hacia una marcada tendencia negativa, con extinción poblacional en el centro de la Comunidad, incluída toda el área urbana y periurbana, donde aparentaba ser una especie común.

\section{Euzonitis haroldi (Heyden, 1870)}

Especie de estatus taxonómico incierto, descrita de Madrid por Heyden (1870) y citada en varias localidades por Rodríguez López-Neyra (1914).

Sus citas, siempre muy escasas, se distribuyen por la mitad oriental de la Comunidad de Madrid, con falta de datos recientes en los alrededores de la ciudad de Madrid (Fig. 6). Todas las citas se concentran entre los 700 y los $800 \mathrm{~m}$ de altitud. Ocupa zonas abiertas esteparias sobre sustrato calizo, aunque la localidad de la que se describió originalmente, la Casa de Campo, se localiza sobre sustratos predominantemente silíceos. Las capturas recientes de ejemplares adultos se efectuaron entre el 28 de mayo y el 15 de junio, sobre flores de Thapsia y Ferula. No disponemos de datos suficientes para evaluar posibles tendencias.

\section{Euzonitis quadrimaculata (Pallas, 1782)}

Especie ampliamente distribuida por el área occidental de la región paleártica (Bologna, 1991), citada en la provincia de Madrid por Graells (1853 sub "Zonitis quadripustulata") y de localidades concretas por Rodríguez López-Neyra (1914, sub Z. auricoma var. bombycina Escherich, 1896).

Sus registros se encuentran dispersos por la mitad oriental de la Comunidad de Madrid. Los datos antiguos se concentraban en los alrededores de la ciudad de Madrid, donde no ha vuelto a ser localizado, mientras que los datos recientes son de áreas calizas del noreste y del sureste (Fig. 6). Sus registros se distribuyen desde los $480 \mathrm{~m}$ de altitud de Aranjuez, hasta cerca de $800 \mathrm{~m}$ al sur de Torrelaguna. Ocupa sobre todo zonas aclaradas, próximas al encinar-quejigar sobre sustrato calizo, aunque los registros antiguos señalan su presencia abundante en encinares silicícolas (por ejemplo: El Pardo, 35 ejemplares; Madrid, 74 ejemplares).

Las capturas recientes de ejemplares adultos se efectuaron entre el 28 de mayo y el 15 de junio. Los adultos se han observado únicamente sobre flores de Thapsia y Ferula. Los registros actuales están constituidos por un menor número de localidades, $\mathrm{y}$ sobre todo un número mucho más bajo de ejemplares observados que los efectuados hace más de 25 años por lo que consideramos que la especie se encuentra en regresión.

\section{Euzonitis sexmaculata (Olivier, 1789)}

Especie euroasiática distribuida por la región mediterránea (Bologna, 1991), citada en la provincia de Madrid por Graells (1853), de la que únicamente se conocen tres ejemplares madrileños (Fig. 6), dos capturados hacia finales de 1800 en Madrid y Villaverde (Madrid) y el otro capturado cien años más tarde en Cercedilla (F.A. Montes, 15-VII-1986) sobre flores de umbelífera. Los dos registros datados corresponden al mes de julio.

Fig. 5.- Mapas de distribución de especies del género Mylabris en la Comunidad de Madrid. Los datos anteriores a 1980 se representan con círculos de color azul.

Fig. 5.- Distribution maps of species of genus Mylabris in Comunidad de Madrid. Observations recorded before 1980 are shown in blue. 
Zonitis (Zonitis) flava Fabricius, 1775

Especie distribuida por gran parte del área sudoccidental de la región paleártica (Bologna, 1991), citada en la provincia de Madrid por Graells (1853) y por Pérez-Moreno et al. (2003).

Sus citas se encuentran dispersas por toda la Comunidad de Madrid, aunque existen grandes lagunas posiblemente por defectos de muestreo (Fig. 6). Los datos antiguos se concentraban en los alrededores de la ciudad de Madrid, mientras que los datos recientes se localizan en áreas calizas del sureste y en terrenos graníticos de la Sierra, con algunas citas en áreas periurbanas. Sus registros se distribuyen desde los $600 \mathrm{~m}$ de altitud de Mejorada del Campo, hasta los $1510 \mathrm{~m}$ en el Puerto de Guadarrama. Ocupa sobre todo zonas abiertas, en todos los pisos de vegetación excepto el oro y el crioromediterráneo.

Las capturas recientes de ejemplares adultos se efectuaron entre el 10 de junio y el 15 de julio. Los adultos se han observado únicamente sobre flores de Eryngium y, aunque se observa con relativa frecuencia, rara vez se ven concentraciones altas de ejemplares sobre un mismo pie de planta. La dispersión de las citas parece indicar que la ausencia de datos es más bien un reflejo de la intensidad de los muestreos que de la presencia de la especie.

\section{Zonitis (Zonitis) immaculata (Olivier, 1789)}

Especie propia de la región mediterránea (Bologna, 1991), citada en Madrid por Rodríguez López-Neyra (1914) y Pérez-Moreno et al. (2003).

Como en el caso anterior sus citas se encuentran dispersas por gran parte de la Comunidad de Madrid, aunque existen grandes lagunas posiblemente por defectos de muestreo (Fig. 6). Los registros se localizan tanto en áreas calizas del sureste, como en terrenos graníticos de la Sierra, y en las áreas de transición, con algunas citas en áreas periurbanas. Sus registros se distribuyen desde los $620 \mathrm{~m}$ de altitud en Villaviciosa de Odón, hasta los $1510 \mathrm{~m}$ en el Puerto de Guadarrama. Ocupa sobre todo zonas abiertas, en todos los pisos de vegetación excepto el oro y el crioromediterráneo.
Las capturas recientes de ejemplares adultos se efectuaron entre el 10 de junio y el 15 de julio. Los adultos se han observado sobre cabezuelas de Centaurea. Se trata de una especie poco frecuente. La dispersión de las citas parece indicar que la ausencia de datos es más bien un reflejo de la intensidad de los muestreos que de la presencia de la especie.

\section{Nemognatha (Nemognatha) chrysomelina (Fabricius, 1775)}

Especie mediterráneo-turánica (Bologna, 1991), no citada previamente en Madrid, sólo representada por un ejemplar de Rivas de Jarama en las colecciones históricas del MNCN, pero que hemos encontrado con relativa frecuencia en zonas yesíferas y calcáreas del sureste (Fig. 7). Los adultos se han encontrado siempre en inflorescencias de Echinops, en zonas relativamente abiertas de atochares con encinas dispersas y quejigares, entre 600 y 800 m de altitud.

\section{Apalus (Criolis) guerini (Mulsant, 1858)}

Especie distribuida por el occidente de la región mediterránea (Bologna, 1991), citada en Madrid por García-París (2000) a partir de ejemplares de la colección del MNCN, de la que únicamente se conoce una observación reciente en el Cerro del Telégrafo, Rivas, el 15 de mayo (R. Sánchez) en una zona yesífera (Fig. 7). Las especies del género Apalus, como las de los demás Sitarini, son difíciles de localizar, y aunque pueden llegar a ser localmente abundantes, sus poblaciones se encuentran muy dispersas.

\section{Sitaris (Sitaris) muralis (Foerster, 1771)}

Especie de amplia distribución en el área sudoccidental de la región paleártica (Bologna, 1991) citada en la provincia de Madrid por Graells (1853, como S. humeralis Westwood, 1839) y de localidades concretas por Torres Sala (1962, sub

Fig. 6.- Mapas de distribución de especies de Mylabrini (Mylabris) y Nemognathini (Euzonitis y Zonitis) en la Comunidad de Madrid. Las cuadrículas de 10x10 km con citas anteriores a 1980 se señalan en azul.

Fig. 6.- Distribution maps of species of Mylabrini (Mylabris) and Nemognathini (Euzonitis and Zonitis) in Comunidad de Madrid. 10x10 km squares including records before 1980 are indicated with blue dots. 

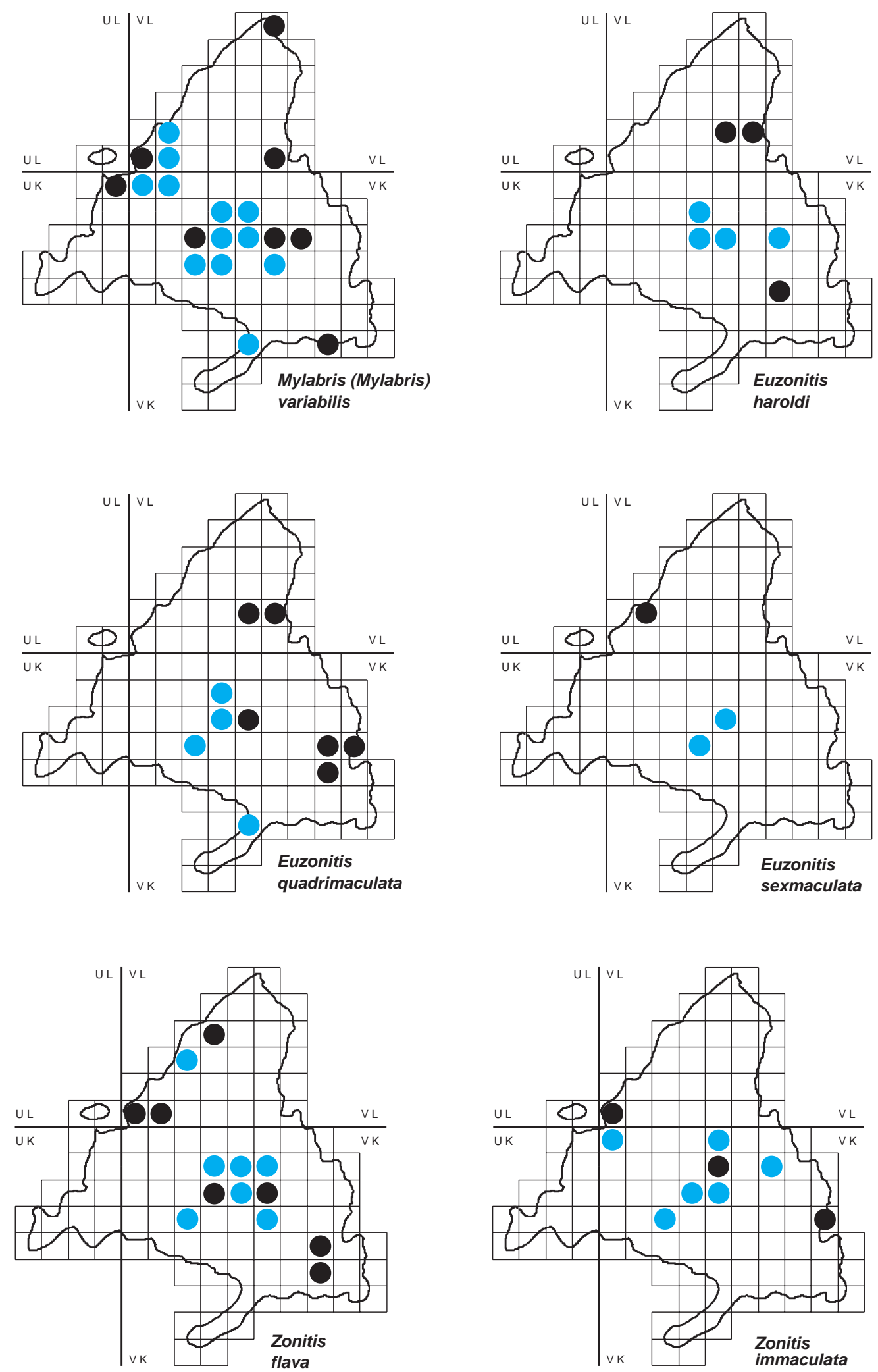
Apalus muralis) y García-París (2000). La presencia recientede la especie en Madrid ha sido documentada en zonas de calizas y yesos del sureste (Fig. 7), Tielmes, 3 de agosto y 5 de septiembre (M. García-París). La presencia en Madrid de otra especie del género, S. solieri Pecchioli, 1839 es posible, ya que se ha encontrado en otras zonas del centro peninsular pero en la actualidad no existe ningún registro.

\section{Stenoria (Stenoria) apicalis (Latreille, 1802)}

Especie ampliamente repartida en el área sudoccidental de la región paleártica (Bologna, 1991), citada en Madrid por Pardo Alcaide (1958), García-París (2000) y Ruiz (2001), sin que exista otra información disponible, ni datos de capturas recientes en los últimos 25 años (Fig. 7). Como ya se comentó en el caso de Apalus, las especies de la tribu Sitarini pasan desapercibidas con facilidad y no existen muestreos específicos (excepto quizás la destrucción de los nidos de sus Apoidea hospedadores) que permitan evaluar realmente la situación de estas especies, que podrían estar mucho mejor distribuidas de lo que los datos permiten estimar.

\section{Conclusiones}

A modo de resúmen final señalamos que de las 40 especies de la familia Meloidae registradas en la Comunidad de Madrid, siete no han vuelto a ser localizadas en los últimos 25 años. Dos de estas siete especies (S. apicalis y $M$. baudueri) están representadas por uno o dos ejemplares y además son de difícil localización, por lo que su presencia actual ha podido pasar desapercibida en los muestreos recientes. Este no es el caso de las otras cinco (Meloe variegatus, M. cavensis, M. violaceus, Mylabris uhagonii y M. amorii), ya que se trata de especies conspícuas, llamativas y representadas por numerosos ejemplares en las colecciones. Los casos más llamativos son los de Meloe variegatus, M. cavensis y Mylabris uhagonii, que cuentan con citas bien distribuidas, y que dada la situación han de ser consideradas En Peligro de Extinción, o tal vez definitivamente extinguidas, si no aparecen nuevos ejemplares en un futuro próximo. El caso de Mylabris amorii, es diferente ya que aunque representado por numerosos ejemplares sólo se ha encontrado en una localidad situada en el límite septentrional de la distribución conocida de la especie, por lo que consideramos que mientras no existan nuevos datos no tenemos criterios suficientes para evaluar su situación. Es posible que Meloe violaceus, por tratarse de una especie preferentemente de montaña que a menudo se encuentra en las mismas zonas que M. proscarabaeus y $M$. brevicollis haya pasado desapercibida entre los ejemplares observados pero no colectados de estas especies. En cualquier caso el retroceso sufrido por esta especie es evidente, y por lo tanto podría considerarse como Vulnerable.

En cinco especies adicionales se ha constatado una reducción evidente de la distribución actual con respecto a la obtenida hace más de 25 años. Esta situación se presenta en Meloe brevicollis, $M$. autumnalis, M. proscarabaeus, Hycleus dufourii y Mylabris dejeani. En todas estas especies la distribución actual se limita a zonas situadas en la Sierra, mientras que el resto de poblaciones parecen haber desaparecido por lo que deberían considerarse en la categoría de Vulnerable. Si continúa la tendencia actual, posiblemente pasarán a la categoría de En Peligro de Extinción. Finalmente hay que destacar el caso de Meloe tuccius, una especie frecuente, que hace tan sólo 10 ó 15 años presentaba la mayoría de las poblaciones conocidas dentro del área urbana de la ciudad de Madrid y sus alrededores, pero que hoy debido a la urbanización y al ajardinamiento pueden considerarse como desaparecidas, aunque esta información no se refleja en los mapas al no haber transcurrido los 25 años utilizados como criterio en la elaboración de éstos; por lo tanto creemos que esta especie ha de incluirse en la categoría de Vulnerable.

Sin duda el grupo que presenta los mayores problemas es el género Meloe. De las 12 especies registradas en Madrid, dos se encuentran en peligro de extinción y cinco claramente amenzadas con pérdida marcada de ocupación, y de las restantes, cuatro son especies que se han encontrado como máximo en tres cuadrículas de $10 \times 10 \mathrm{~km}$. Por lo tanto, la única especie que parece mantenerse en una situación estable en Madrid es Meloe mediterraneus, que aunque tampoco presenta poblaciones numerosas, al menos se conocen citas en siete cuadrículas dispersas, tres de ellas con presencia reciente.

Finalmente no podemos concluir sin indicar que aunque no disponemos de datos cuantitativos, las apreciaciones obtenidas durante los muestreos sugieren que incluso las especies más abundantes, como Berberomeloe majalis, son mucho menos frecuentes en los últimos años. Sin entrar a discu- 

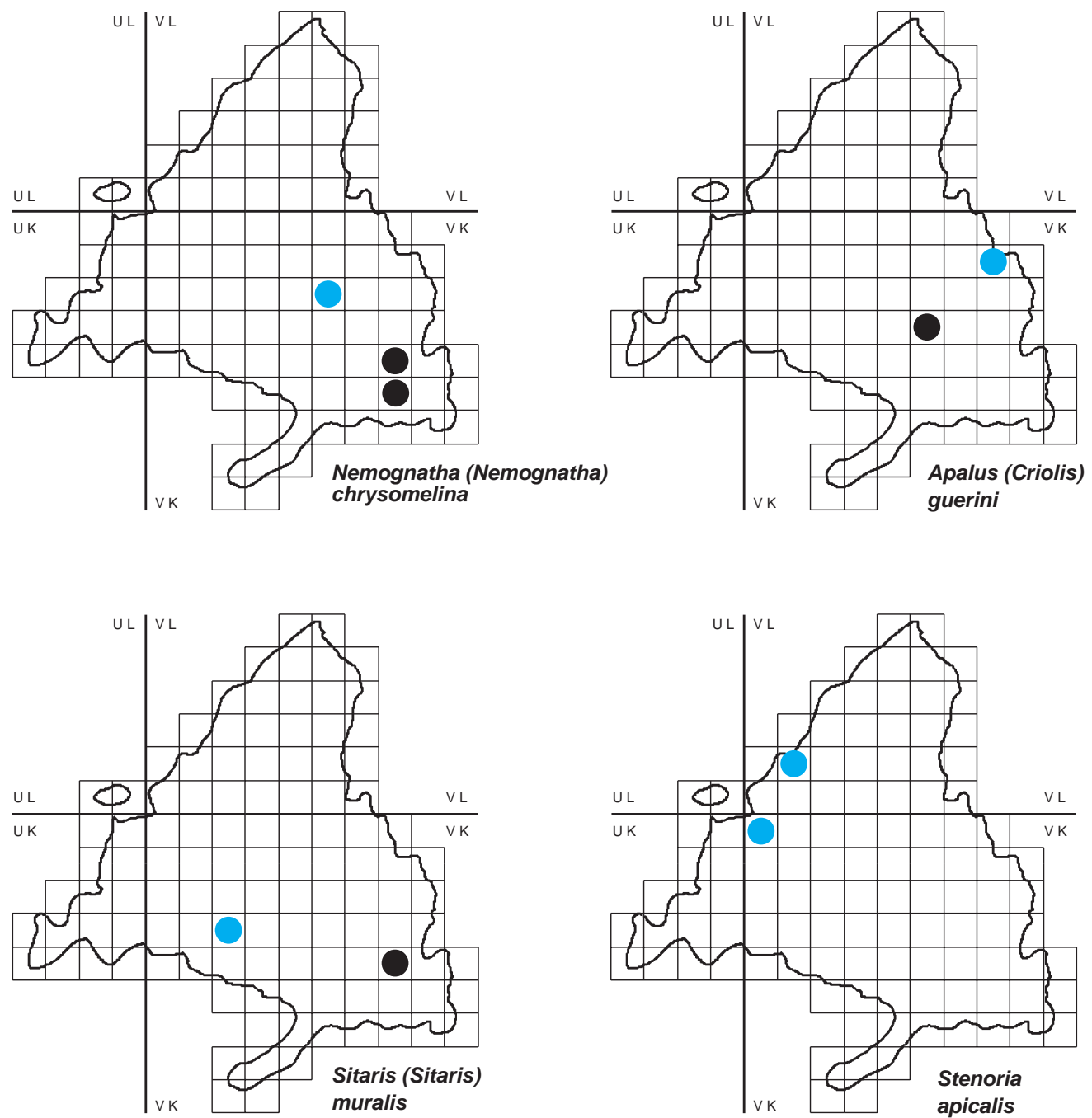

Fig. 7.- Mapas de distribución de especies de Nemognathini (Nemognatha) y Sitarini (Apalus, Sitaris y Stenoria) en la Comunidad de Madrid. Las cuadrículas de 10x10 km con citas anteriores a 1980 se señalan en azul.

Fig. 7.- Distribution maps of species of Nemognathini (Nemognatha) and Sitarini (Apalus, Sitaris and Stenoria) in Comunidad de Madrid. 10x10 km squares including records before 1980 are indicated with blue dots.

tir el tema de la urbanización que acaba con poblaciones completas, el uso de herbicidas en las cunetas, el empleo masivo de productos fitosanitarios en los cultivos, campos de golf y jardines y, las fumigaciones aéreas contra plagas locales, son en la actualidad problemas muy serios para las poblaciones de invertebrados. La disminución de los meloidos, por ser parasitoides de abejas y saltamontes, sugiere una evidente disminución de las poblaciones de sus huéspedes, por lo que el problema actual ha de considerarse a una escala de ecosistema y no sólo de grupos taxonómicos concretos como el que nos ocupa. Nuestras apreciaciones en este sentido son pesimistas, pero con los datos presentados en este trabajo tendremos oportunidad de comprobar, qué ha ocurrido con las poblaciones actuales de Meloidae de Madrid en los próximos años. 


\section{AGRADECIMIENTOS}

Los trabajos sobre el estado de conservación de faunas regionales no pueden realizarse sin el concurso de todos aquellos entomólogos profesionales y aficionados que durante años han depositado sus colecciones en Instituciones públicas de libre acceso a la comunidad científica. Este trabajo se ha podido relizar gracias a todas estas personas que desde finales de 1800 contribuyeron a la formación de colecciones, auténticas bases de datos del pasado consultables "en tiempo real" que a la vez constituyen la fuente de datos y los datos en sí mismos. A todos ellos nuestro agradecimiento por dejarnos "ver" cómo era la fauna de Madrid desde hace más de un siglo. Agradezco a los siempre dispuestos y desinteresados colegas L. Arnáiz, P. Bercedo, J.I. López-Cólon, F.A. Montes, R. Sánchez, M. A. S. Sobrino y J. L. Zapata su amabilidad cediendonos material para estudio y permitiendonos revisar sus colecciones; a los conservadores e investigadores de los museos visitados, O. Merkl y J. Vörös en Budapest, B. Jordal en el Museo de la Universidad NTNU en Trondheim, P. Oromí y M. Báez en La Laguna, I. Izquierdo, M. París y C. Martín en Madrid; a G. Astudillo, G. García Martín, J. Hanken, G. Parra Olea, R. López, I. Martínez-Solano, F.A. Montes, A. Perdices, M. Pola, E. P. Zapirain, y R. Zardoya, que nos acompañaron en muestreos o nos proporcionarnos valioso material de campo; y especialmente a J.L. Ruiz y M.A. Alonso Zarazaga por su apoyo, críticas y saber hacer científico.

Este trabajo ha contado para su realización con los fondos de los proyectos GR/AMB/0750/2004 de la Comunidad de Madrid y CGL2004-04680-C10-10/BOS del Ministerio de Educación y Ciencia. El primero nos ha permitido realizar revisiones de colecciones, muestreos y análisis moleculares, y el segundo ha facilitado el apoyo logístico para el desarrollo del trabajo (desde aquí nuestro agradecimiento a P. Fernández por su imprescindible labor). Agracedemos a la Comunidad de Madrid los permisos necesarios para la realización de los muestreos de campo. La visita a la colección del Magyar Természettudomány Múzeum en Budapest ha sido financiada por el proyecto Synthesys «European Commission's Research Infrstructure Action».

\section{Referencias}

AMOR MaYor, F., 1860. Memoria sobre los insectos epispásticos de algunas provincias de España: presentada al Colegio de Farmaceuticos de Madrid. Impr. Manuel Álvarez, Madrid. 36 pp.

BolognA, M. A., 1988. Note su Eurymeloe e revisione delle specie euromediterranee del gruppo rugosus (Coleoptera, Meloidae). Fragmenta Entomologica, 20(2): 233-301.

BolognA, M. A., 1989. Berberomeloe, a new west Mediterranean genus of Lyttini for Meloe majalis Lineé (Coleoptera, Meloidae). Systematics and bionomics. Bolletino di Zoologia, [1988], 55: 359-366.

Bologna, M. A., 1991. Fauna de Italia. Coleoptera Meloidae. Calderini, Bologna. 541 pp.

Bologna, M. A. \& Aloisi, G., 1994. Systematics and bionomics of Physomeloe Reitter, 1911, with description of the first instar larva (Coleoptera, Meloidae). Eos, [1993], 69: 45-56.
Bologna, M. A. \& Pinto, J. D., 2001. Phylogenetic studies of Meloidae (Coleoptera) with emphasis on the evolution of phoresy. Systematic Entomology, 26: 33-72.

Bologna, M. A. \& Pinto, J. D., 2002. The Old World genera of Meloidae (Coleoptera): a key and synopsis. Journal of Natural History, 36(17): 2013-2102.

Borchmann, F., 1917. Pars 69. Meloidae, Cephaloidae. In: E. Schenkling (ed.). Coleopterorum Catalogus auspiciis et auxilio W. Junk. Junk. Berlin. 208 pp.

Capote, L. \& García-París, M., 2001. Distribución geográfica de los Cerocomini ibéricos (Coleoptera: Meloidae). Boletín de la Asociación Española de Entomología, 25: 31-44.

De la Fuente, J. M., 1933. Catálogo sistemático geográfico de los Coleópteros observados en la Península Ibérica, Pirineos propiamente dichos y Baleares. Boletín de la Sociedad entomológica de España, 16: 18-32; 45-49.

DíAz LizAnA, R., 1864. Consideraciones filosófico-naturales sobre los insectos, seguidas de una Monografia de los epispásticos. Impr. Manuel Tello, Madrid. 42 pp.

DIECK, G., 1870. Eine entomologische Wintercampagne in Spanien. Berliner entomologische Zeitschrift, 14: 145-184.

FLACH, K., 1907. Beiträge zur Käferfauna der Iberische Halbinsel. Wiener entomologische Zeitung, 26: 17-19.

GARCíA-PARís, M., 1998. Revisión del género Berberomeloe Bologna, 1988 (Coleoptera: Meloidae) y diagnosis de un endemismo ibérico olvidado. Graellsia, 54: 97-109.

GARCÍA-PARís, M., 2000. Nuevas localidades de Sitarini (Col. Meloidae) de la Península ibérica. Boletín de la Asociación Española de Entomología, 24: 197-200.

García-París, M., Ruiz, J. L. \& París, M., 2003. Los representantes de la tribu Lyttini (Coleoptera: Meloidae) de la Península Ibérica. Graellsia, 59(23): 69-90.

GarcíA-PArís, M. \& Ruiz, J. L., 2005. Bibliografía y registros ibero-baleares de Meloidae (Coleoptera) publicados hasta la aparición del "Catálogo sistemático geográfico de los Coleópteros observados en la Península Ibérica, Pirineos propiamente dichos y Baleares" de J. M. de la Fuente (1933). Graellsia, 61(2): 225-255.

GÓmEZ CARRASCO, E., 1888. Excursión a San Ildefonso por Peñalara. Anales de la Sociedad Española de Historia natural, 17: 63-66.

GóRriz MuÑoz, R. J., 1882. Ensayo para la monografía de los coleópteros melóidos indígenas con aplicación a las ciencias médicas. Imprenta y librería de J. Sanz, Zaragoza. 199 pp., 2 láms.

Graells, M. P., 1849. Description de coléoptères nouveaux propres à la Faune centrale de l'Espagne. Revue et Magazine de Zoologie, 1849: 621-622. 
Graells, M. P., 1851a. Description de quelques coléoptères nouveaux de la Faune centrale d'Espagne. Annales de la Société Entomologique de France, série 2, 9: 5-27, pl.1.

Graells, M. P., 1851b. Descripción de algunos insectos nuevos pertenecientes a la fauna central de España. Memorias de la Real Academia de Ciencias de Madrid, serie 3, [1850], 1(2): 109-163, pl. 8-10.

Graells, M. P., 1853. “Sección Zoolójica” págs. 36-86. En: Luján, F. de (ed.) Memoria que comprende el resumen de los trabajos verificados en el año de 1852 por las diferentes secciones encargadas de formar el Mapa Geológico de la provincia de Madrid y el General del Reino presentada en 11 de Junio de 1853 al Excmo. Señor Ministro de Fomento. Imprenta de Eusebio Aguado, Madrid. 88 pp., 1 tab., 3 láms.

HaVelKA, P., 1980. Eine interessante Ölkäferart (Meloe violaceus) (Coleoptera, Meloinae) an einem Trockenstandort im Rheinvorland bei Eggenstein. Pfälzer Heimat, 31: 110-112.

HeYden, L. von, 1870. Entomologische reise nach dem südlichen Spanien, der Sierra de Guadarrama und Sierra Morena, Portugal und den Cantabrischen Gebirgen, mit Bescreibungen der neuen Arten. Herausgegeben von dem entomologischen Vereine. Nicolai'sche Verlagsbuchhandlung. Berlin. pp: 1175. (Este trabajo también se publicó como suplemento del Berliner entomologische Zeitschrift).

LÓPEZ-ColÓN, J. I., 1991. Nuevas localizaciones de algunos coleópteros ibéricos. Nouvelle Revue d'Entomologie (N.S.), 8(3): 265.

LÓPEZ-ColÓN, J. I., 2005. La Naturaleza en Rivas: Los milabrinos. Zarabanda (Rivas-Vaciamadrid), 64: 12.

MAder, L., 1927. Meloidae. In: Winkler, A. (ed.). Catalogus Coleopterorum regionis palaearcticae, II. Winkler. Wien: 851-888.

Marseul, S.A. DE, 1870. Monographie des Mylabrides d'Europe et des contrées limitrophes en Afrique et en Asie. L'Abeille, 7(2e partie): 1-204.

Martínez SÁEz, F. P., 1873. Datos sobre algunos coleópteros de los alrededores de Cuenca. Anales de la Sociedad Española de Historia Natural, 2: 53-75.

Martínez SÁEz, F. P., 1883. Sin título [Coleópteros de España recolectados por Don M. Laguna]. Actas de la Sociedad Española de Historia Natural, 12: 1832.

Martínez-Solano, I. \& GonzÁlez Fernández, J. E., 2003. La colección de anfibios de Madrid del Museo Nacional de Ciencias Naturales y su utilidad en conservación. Graellsia, 59(2-3): 105-128.

NASH, D., 1976. Some interesting Coleoptera from Northeast Essex, England. The Entomologist's Record and Journal of Variation, 88: 39-43.
Ortuño, V. M. \& Hernández, J. M., 1993. Diversos casos teratológicos en Coleoptera. Boletín de la Real Sociedad Española de Historia Natural (Sección Biológica), 89: 163-179.

PARdo Alcaide, A., 1950. Estudios sobre Meloidae II. Los Mylabrini de la Península Ibérica. Boletín de Patología Vegetal y Entomología Agrícola, [1949], 17: 61-82.

Pardo Alcaide, A., 1952. Los géneros de Meloidae de la fauna hespérica. Graellsia, [1950], 8: 39-79.

Pardo Alcaide, A., 1956a. Analectas entomológicas. V. Los Zonitis de la Fauna Ibérica (Col. Meloidae). Graellsia, 14: 13-19.

Pardo Alcaide, A., 1956b. Los Cerocoma de la fauna ibérica (Col. Meloidae). Graellsia, 14: 21-27.

Pardo Alcaide, A., 1958. Los Sitarini de la Península Ibérica (Col. Meloidae). Graellsia, 16: 13-22.

Pérez-Moreno, I., San Martín, A. F. \& Recalde IRURZUN, J. I., 2003. Aportaciones corológicas y faunísticas sobre meloidos ibéricos (Coleoptera: Meloidae). Boletín de la Sociedad Entomológica Aragonesa, 33: 195-217.

Pleguezuelos, J. M., 1991. Evolución histórica de la avifauna nidificante en el S.E. de la Península Ibérica (1850-1985). A.M.A. Consejería de Cultura y Medio Ambiente. Junta de Andalucía. Sevilla. 61 pp.

Rodríguez LóPEZ-NEYrA, C., 1914. Claves dicotómicas para la determinación de los meloideos indígenas. Boletín de la Real Sociedad Española de Historia Natural, 14: 461-475.

Ruiz, J. L., 2001. Las Stenoria Mulsant, 1857 del grupo de $S$. apicalis (Latreille, 1802) de la Península Ibérica (Coleoptera, Meloidae). Graellsia, 57(2): 161-173.

SAnZ DE Diego, M., 1880. Sin título [Nota sobre algunos insectos de España]. Actas de la Sociedad Española de Historia Natural, 9: 38-39.

SEIDlitz, G., 1867. Einige entomologische Excursionen in den Castilischen Gebirgen im Sommer 1865. Berliner entomologische Zeitschrift, 11: $167-178$

Shaffer, H. B., Fisher, R. N. \& Davidson, C., 1998. The role of natural history collections in documenting species declines. Trends in Ecology and Evolution, 13(1): 27-30.

Sumakov, G., 1930. Catalogue des espèces paléarctiques de tribu Mylabrina (Coleoptera, Meloidae). Tartu Ülikooli juures oleva Loodusuurijate Seltsi Aruanded, 37(1-2): 1-114.

Sumakov, G., 1934. Inventa Entomologica itineris Hispanici et Maroccani, quod a. 1926 fecerunt Harald et Hakan Lindberg. XXI. Meloides du Bassin Méditerranéen et du Maroc. Societas Scientiarum Fennica, Commentationes Biologicae, 4(13): 1-6. 
TeXIDOR Cos, J., 1880. Apuntes de zoología médico-farmaceútica y farmaco-zoología. Imprenta de Oliveres. Barcelona. $1014+1$ pp.

Torres Sala, J. DE. 1962. Catálogo de la colección entomológica "Torres Sala" de coleópteros y lepidópteros de todo el Mundo. Vol. 1. Instituto Alfonso El Magnánimo. Diputación Provincial de Valencia. Valencia. 487 pp.

Traizet, E., 1895. Sin título. Actas de la Sociedad Española de Historia Natural, 24: 183-184.
TraIZET, E., 1896. Observations biologiques sur le Notoxus monoceros (Col.). Bulletin de la Societé Entomologique de France, 1896: 104.

Trotta-Moreu, N. \& García-París, M., 2001. Los Hycleus (Coleoptera: Meloidae) de la Península Ibérica: distribución geográfica y variabilidad elitral. Graellsia, 57: 99-111.

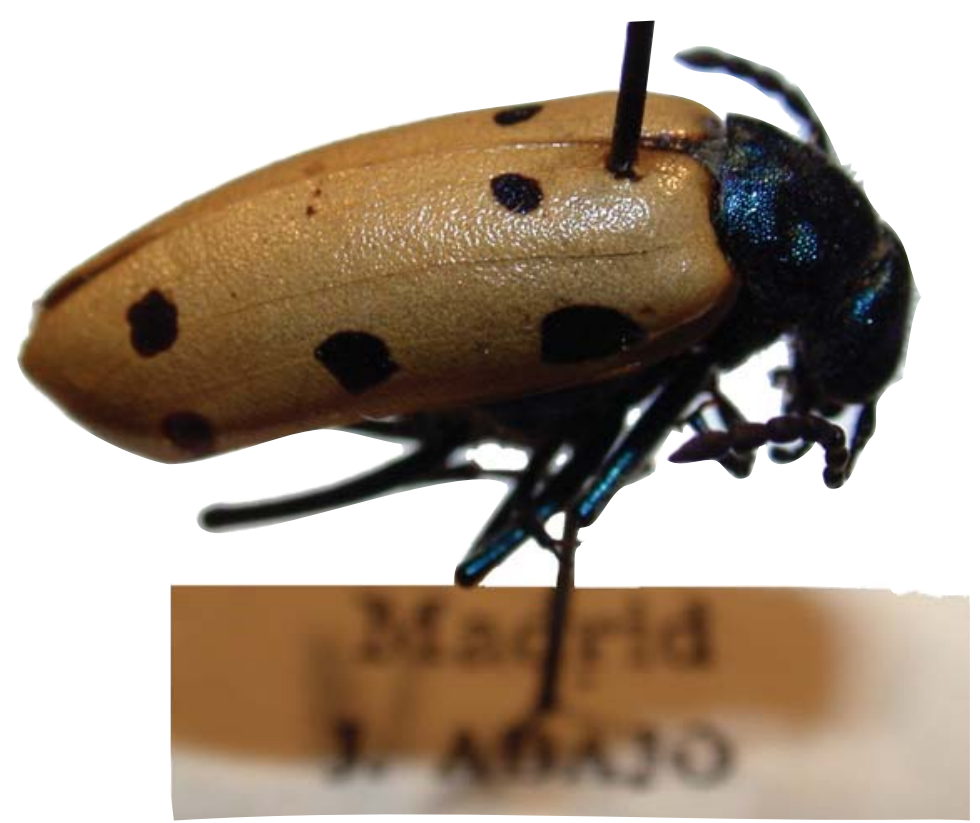

Mylabris (Chalcabris) uhagonii Martínez Sáez, 1873. 
Apéndice 1.- Citas publicadas de Meloidae en la Comunidad de Madrid

Appendix 1.- - Published records of Meloidae from Comunidad de Madrid.

Cerocoma (Cerocoma) schaefferi (Linnaeus, 1758)

Aranjuez (Capote \& García-París, 2001)

Arganda (Capote \& García-París, 2001)

Cantoblanco (Capote \& García-París, 2001)

El Escorial (Górriz Muñoz, 1882; Capote \& García-París, 2001)

El Escorial (Rodríguez López-Neyra, 1914: sub C. wahlii var. chalybeiventris)

El Escorial: Fuente de la Teja (Capote \& García-París, 2001)

El Escorial: Los Arroyos (Capote \& García-París, 2001)

El Pardo (Capote \& García-París, 2001; Pérez-Moreno et al., 2003)

El Pardo (Rodríguez López-Neyra, 1914: sub C. wahlii var. chalybeiventris)

Guadarrama (Capote \& García-París, 2001)

La Navata (Capote \& García-París, 2001)

Lozoya (Capote \& García-París, 2001)

Madrid [UTM Puerta del Sol] (Górriz Muñoz, 1882;

Capote \& García-París, 2001)

Madrid: [Alameda de] Osuna (Capote \& García-París,a 2001)

Madrid: Casa de Campo (Capote \& García-París, 2001)

Madrid: Dehesa de la Villa (Capote \& García-París, 2001)

Montarco [Rivas de Jarama] (Capote \& García-París, 2001)

Montejo de la Sierra (Capote \& García-París, 2001)

Puerto de Cotos (Capote \& García-París, 2001)

Puerto de Navacerrada (Capote \& García-París, 2001)

Ribas [Cristo de] (Capote \& García-París, 2001)

San Sebastián de los Reyes (Capote \& García-París, 2001)

Sierra de Guadarrama [UTM de Guadarrama]

(Capote \& García-París, 2001)

Vaciamadrid (Capote \& García-París, 2001)

Villaviciosa de Odón (Capote \& García-París, 2001)

Cerocoma (Metacerocoma) schreberi Fabricius, 1781

Cercedilla (Capote \& García-París, 2001)

El Escorial (Martínez Sáez, 1883)

El Escorial (Capote \& García-París, 2001; Pérez-Moreno et al., 2003)

El Escorial, Fuente de la Teja (Capote \& García-París, 2001)

El Escorial, Los Arroyos (Capote \& García-París, 2001)

Guadarrama (Capote \& García-París, 2001)

Lozoya (Capote \& García-París, 2001)

Madrid: El Pardo (Capote \& García-París, 2001)

Madrid: Casa de Campo (Capote \& García-París, 2001)

Madrid: Moncloa (Capote \& García-París, 2001)

Montarco [Rivas de Jarama] (Capote \& García-París, 2001)

Embalse de Valmayor (Capote \& García-París, 2001)

Villalba (Capote \& García-París, 2001)

Villaviciosa de Odón (Capote \& García-París, 2001)

Vaciamadrid (Pérez-Moreno et al., 2003)

Berberomeloe majalis (Linnaeus, 1758)

$1 \mathrm{~km}$ al SO de Valdaracete, $800 \mathrm{~m}$ (García-París et al., 2003)

$2 \mathrm{~km}$ al E de Valdilecha (García-París et al., 2003)

2 km al SE de Arganda, 700 m (García-París, 1998;

García-París et al., 2003)

$3 \mathrm{~km}$ al E de Valdaracete (García-París et al., 2003)

$3 \mathrm{~km}$ al NO de Camporreal, $700 \mathrm{~m}$ (García-París, 1998; García-París et al., 2003)

$3 \mathrm{~km}$ al $\mathrm{O}$ de Villamanrique de Tajo, $580 \mathrm{~m}$ (García-París et al.,2003) VK7736

$3 \mathrm{~km}$ al S de Arganda, $680 \mathrm{~m}$ (García-París, 1998

García-París et al., 2003)

$3 \mathrm{~km}$ al S de Valdaracete, $790 \mathrm{~m}$ (García-París et al., 2003)

$4 \mathrm{~km}$ al N de Villarejo de Salvanés (García-París et al., 2003)

$4 \mathrm{~km}$ al S de Pozuelo del Rey, 800 m (García-París et al., 2003)

4 km al SE de Arganda, 720 m (García-París, 1998;

García-París et al., 2003)

$5 \mathrm{~km}$ al NO de Carabaña (García-París et al., 2003)

Arganda (García-París, 1998)

Camporreal - Loeches (García-París et al., 2003)

Casa de la Aldehuela, $5 \mathrm{~km}$ al SO de Torrelaguna, $790 \mathrm{~m}$ (García-París et al., 2003)
Cercedilla (García-París, 1998)

VL11

Chinchon (García-París, 1998)

Cobeñas [UTM de Cobeña] (García-París, 1998) VK59

El Berrueco (García-París et al., 2003) VL52

El Molar (García-París, 1998) VL50

Hayedo de Montejo - Puerto del Cardoso (García-París et al., 2003) VL54

Loeches (García-París, 1998) VK67

Madrid (Bologna, 1989, García-París, 1998) VK47

$\begin{array}{ll}\text { Madrid, alrededores (Górriz Muñoz, 1882; Flach, 1907) } & \text { VK47 }\end{array}$

Madrid, Aluche (García-París, 1998)

Madrid, Aluche (Pérez-Moreno et al., 2003)

Madrid, Canal [UTM Canal de Isabel II] (García-París, 1998) VK47

Madrid, Cerro Negro [Vallecas] (Rodríguez López-Neyra, 1914; García-París, 1998)

Madrid, Cerro Almodóvar (Pérez-Moreno et al., 2003) VK47

Madrid, Ciudad Universitaria (García-París, 1998) VK3878

Madrid, Dehesa de la Villa (García-París, 1998; García-París et al., 2003) VK37

Madrid, El Pardo (García-París, 1998) $\quad$ VK38

Madrid, Hortaleza (García-París et al., 2003) V VK48

Madrid, Moncloa (García-París, 1998) VK37

Madrid, Santa Eugenia, Cerro Almodóvar (García-París, 1998) VK4871

Madrid, Vallecas (García-París, 1998) VK46

Madrid, Vallecas, Cerro Almodóvar (García-París, 1998) $\quad$ VK47

Madrid, Vicálvaro (Sumakov, 1934; García-París, 1998) _ VK47

Madrid, Villaverde (García-París, 1998) VK36

$\begin{array}{ll}\text { Mataelpino (García-París et al., 2003) } & \text { VL20 }\end{array}$

Montejo de la Sierra, 1150m (García-París, 1998) VL54

Moralzarzal (García-París, 1998)

Paracuellos del Jarama (García-París, 1998) VK58

Puerto de Somosierra (Pérez-Moreno et al., 2003) VL55

Ribas [UTM Cristo de Ribas] (García-París, 1998) VK57

Rivas (Traizet, 1895, 1896)

Rivas de Jarama (García-París, 1998) VK56

Rivas, Montarco (García-París, 1998) VK56

Sierra de Guadarrama [UTM de Guadarrama] (García-París, 1998) VL00

Soto del Real-Colmenar Viejo (García-París, 1998) VL30

Universidad Autónoma (Pérez-Moreno et al., 2003) VK49

Vaciamadrid (García-París, 1998) — VK56

Lytta (Lytta) vesicatoria (Linnaeus, 1758)

4 km S Robledo de Chavela, base Pico Almenara

(García-París et al., 2003)

$5 \mathrm{~km}$ al N de Cercedilla (García-París et al., 2003)

Becerril de la Sierra (Pérez-Moreno et al., 2003)

Cercedilla (García-París et al., 2003)

Cercedilla (Pérez-Moreno et al., 2003)

Cercedilla: El Ventorrillo, $1480 \mathrm{~m}$ (García-París et al., 2003)

Cercedilla: Estación Alpina, 1500 m (García-París et al., 2003)

Cercedilla: La Peñota (García-París et al., 2003)

$\begin{aligned} & \text { VK8250 } \text { El Berrueco (García-París et al., 2003) } \\ & \text { VK76 El Escorial (Górriz Muñoz, 1882; García-París et al., 2003) }\end{aligned}$

El Escorial: Fuente de la Teja (García-París et al., 2003)

El Escorial: Urbanización Los Arroyos (García-París et al., 2003)

El Ventorrillo (Pérez-Moreno et al., 2003)

a Pedriza (García-París et al., 2003)

Madrid (Díaz Lizana, 1864; Górriz Muñoz, 1882;

García-París et al., 2003)

Manzanares (Pérez-Moreno et al., 2003)

Montejo de la Sierra (García-París et al., 2003)

Navalquejigo (García-París et al., 2003)

Puerto de Somosierra (García-París et al., 2003)

Rascafría (Pérez-Moreno et al., 2003)

Rascafría: Camino Laguna de los Pájaros (García-París et al., 2003)

Robledo de Chavela (García-París et al., 2003)

Rozas de Puerto Real (García-París et al., 2003)

Somosierra (García-París et al., 2003)

Valdemanco (García-París et al., 2003)

VL5415 Villalba (García-París et al., 2003)
UK9380

VL11

VL10

VL11

VL11

VL11

VL11

VL01

VL52

VK09

VK09

09

L11

VK47

VL20

VL54

VK19

VL55

VL22

VL22

UK98

UK76

VL55

VL42

VK19 
Oenas fusicornis Abeille de Perrin, 1880

$2 \mathrm{~km}$ al E de Carabaña, 600 m (García-París et al., 2003)

$2 \mathrm{~km}$ al S de Tielmes (García-París et al., 2003)

(Garcia-París et al., 2003) VK8084

$3 \mathrm{~km}$ al NE de Olmeda de las Fuentes, $820 \mathrm{~m}$ (García-París et al., 2003) VK8371

$3 \mathrm{~km}$ al O de Pezuela de las Torres, $870 \mathrm{~m}$ (García-París et al., 2003) VK8374

$3 \mathrm{~km}$ al O de Valdilecha, $790 \mathrm{~m}$ (García-París et al., 2003) VK7160

$3 \mathrm{~km}$ al SE de Torres de la Alameda, $760 \mathrm{~m}$ (García-París et al., 2003) VK7071

$3 \mathrm{~km}$ al SE de Villar del Olmo, $780 \mathrm{~m}$ (García-París et al., 2003) VK8164

$5 \mathrm{~km}$ al N de Tielmes (García-París et al., 2003)

$5 \mathrm{~km}$ al O de Valdilecha, $750 \mathrm{~m}$ (García-París et al., 2003)

Alto del Chaparral, Algete (García-París et al., 2003)

Aranjuez (García-París et al., 2003)

Arroyo de la Vega, $1 \mathrm{~km}$ al $\mathrm{O}$ de Olmeda de las Fuentes

(García-París et al., 2003)

Casa de la Aldehuela, $5 \mathrm{~km}$ al SO de Torrelaguna (García-París et al., 2003) VL51

Cercedilla: Estación Alpina (García-París et al., 2003)

El Escorial (García-París et al., 2003)

Fresno de Torote (García-París et al., 2003)

Getafe (García-París et al., 2003)

Loeches (García-París et al., 2003)

Los Santos de la Humosa, 820 m (García-París et al., 2003)

Madrid (Rodríguez López-Neyra, 1914: sub O. afer)

Madrid (García-París et al., 2003) [UTM Puerta del Sol]

Montarco (García-París et al., 2003)

Nuevo Baztán (García-París et al., 2003)

Perales de Tajuña (García-París et al., 2003)

Ribatejada (García-París et al., 2003)

Tielmes (García-París et al., 2003)

Torrelaguna - El Vellón (García-París et al., 2003)

Valdelaguna, $730 \mathrm{~m}$ (García-París et al., 2003)

Venturada (García-París et al., 2003)

Villaviciosa de Odón (García-París et al., 2003)

VK6959

VK5

VK43

VL11

VK09

VK69

VK36

VK6

K7883

VK47

VK47

VK56

VK56
VK76

VK76

VL60

VK75

VL5

VK6845

VL4

VK26

Physomeloe corallifer (Germar, 1818)

$2 \mathrm{~km}$ al O de La Cabrera, 1100 m (García-París et al., 2003)

$2 \mathrm{~km}$ al S de Valdemanco, $1000 \mathrm{~m}$ (García-París et al., 2003)

Alcalá de Henares (García-París et al., 2003)

Alto del Chaparral, Algete (García-París et al., 2003)

Becerril de la Sierra (García-París et al., 2003)

Canto Blanco (García-París et al., 2003)

Cercedilla (Bologna \& Aloisi, 1994; García-París et al., 2003)

Cercedilla (Pérez-Moreno et al., 2003)

Cercedilla: La Peñota (García-París et al., 2003)

Cerro de Los Ángeles (García-París et al., 2003: sólo indicada la cuadrícula UTM)

Cerro San Pedro (García-París et al., 2003)

Chinchón (García-París et al., 2003)

Cienvallejos, Brunete (Bologna \& Aloisi, 1994; García-París et al., 2003)

Collado Mediano (Bologna \& Aloisi, 1994)

Collado Mediano (García-París et al., 2003)

El Escorial (Sanz de Diego, 1880; Bologna \& Aloisi, 1994;

García-París et al., 2003

El Escorial (Pérez-Moreno et al., 2003)

El Ventorrillo, Cercedilla (García-París et al., 2003)

Ermita del Espino, Daganzo (García-París et al., 2003)

Fuente la Teja, El Escorial (García-París et al., 2003)

Gózquez de Abajo (García-París et al., 2003)

Guadarrama (Bologna \& Aloisi, 1994)

Guadarrama (Pérez-Moreno et al., 2003)

Guadarrama: Alto del León (García-París et al., 2003)

La Pedriza de Manzanares (García-París et al., 2003)

Las Rozas (Graells, 1853; García-París et al., 2003)

Los Molinos (García-París et al., 2003)

Los Roncajales (García-París et al., 2003)

Lozoya (Bologna \& Aloisi, 1994; García-París et al., 2003)

Madrid (Torres Sala, 1962) [UTM Puerta del Sol]

Madrid (Bologna \& Aloisi, 1994; García-París et al., 2003)

[UTM Puerta del Sol]

Madrid, alrededores (Flach, 1907) (citado como M. c. var. evae)

Madrid (Górriz Muñoz, 1882) [UTM Puerta del Sol]

Madrid, Campamento (Pérez-Moreno et al., 2003)

Madrid, Chamartín (Pérez-Moreno et al., 2003)

Madrid, Los Pinos-Carabanchel (Pérez-Moreno et al., 2003)

Madrid, Vicálvaro (Sumakov, 1934)

Madrid: Canal (García-París et al., 2003)

Madrid: Cerro Almodóvar (García-París et al., 2003)

VL4623

VL4422

VK68

VK59

VK48

VL1

VL1

VLO

VK46

VL3

VK64

VK17

VL10

VL10

VK09

VK09

VL11
VK68

VK0

VK55

VLOO

VL00

VLO0

VL2

VK28

VL30

VL3

VK47

VK47

VK47

VK4

VK37

VK48

VK36

VK4

VK47

VK47
Madrid: Chamartín (García-París et al., 2003)

Madrid: Ciudad Universitaria (García-París et al., 2003)

Madrid: Dehesa de la Villa (García-París et al., 2003)

Madrid: El Pardo (García-París et al., 2003)

La Fortuna [Leganés] (García-París et al., 2003)

Madrid: Valdebernardo, Vicálvaro (García-París et al., 2003)

Madrid: Vallecas (García-París et al., 2003)

Madrid: Virgen del Cortijo (García-París et al., 2003)

Manjirón, VL5136 (García-París et al., 2003)

Miraflores de la Sierra (Bologna \& Aloisi, 1994;

García-París et al., 2003)

Navacerrada (Dieck, 1870; Bologna \& Aloisi, 1994)

Navacerrada (García-París et al., 2003)

Patones (Ortuño \& Hernández, 1993)

Peña Pintada, Navacerrada (García-París et al., 2003)

Pradera de Navalurraque, Cercedilla (García-París et al.,2003)

Puerto de la Fuenfría, Cercedilla (García-París et al., 2003)

Puerto de la Morcuera (García-París et al., 2003)

Puerto de la Morcuera: 1600 m (García-París et al., 2003)

VL22

Rivas (Traizet, 1896) VK57

Orillas del Jarama [Rivas de Jarama] (Graells, 1853) VK56

Sierra de Guadarrama, Puerto de la Morcuera (Bologna \& Aloisi, 1994) VL22

Valdemanco de la Sierra (García-París et al., 2003)

Villaviciosa de Odón (Pérez-Moreno et al., 2003) VK26

Meloe (Coelomeloe) tuccius (Rossi, 1792)

Madrid: Aluche (Pérez-Moreno et al., 2003)

VLO0
Madrid: Campamento (Pérez-Moreno et al., 2003)

Madrid: Vicálvaro (Sumakov, 1934)

Madrid: Deh. [Dehesa de la] Villa (Torres Sala, 1962)

Madrid (Górriz Muñoz, 1882)

Madrid: Casa de Campo (Górriz Muñoz, 1882)

Rivas (Traizet, 1896)

Meloe (Eurymeloe) baudueri Grenier, 1863

Madrid (Pérez-Moreno et al., 2003)

VK37

VK37

VK47

VK47

VK47

VK37

VK57

Meloe (Eurymeloe) brevicollis Panzer, 1793

Somosierra (Pérez-Moreno et al., 2003)

VK47

Meloe (Eurymeloe) mediterraneus G. Müller, 1925

Madrid (Rodríguez López-Neyra, 1914, citada como M. rugosus) VK47

Madrid, Campamento (Pérez-Moreno et al., 2003)

VK37

Meloe (Eurymeloe) murinus Brandt et Erichson, 1832

Madrid (Rodríguez López-Neyra, 1914)

VK47

Meloe (Meloe) proscarabaeus Linnaeus, 1758

Madrid, Carabanchel (Pérez-Moreno et al., 2003)

Sierra de Guadarrama (Pérez-Moreno et al., 2003)

VK36

Meloe (Meloe) violaceus Marsham, 1802

Madrid, Chamartín (Pérez-Moreno et al., 2003)

Puerto de Malagón (Pérez-Moreno et al., 2003)

Meloe (Treiodus) autumnalis Olivier, 1792

Las Rozas (Graells 1853, sub Meloe cyaneus)

VK28

Actenodia billbergi (Gyllenhal, 1817)

El Escorial (Pérez-Moreno et al., 2003)

Madrid (Rodríguez López-Neyra, 1

Madrid (Sumakov, 1930, sub Hycleus bilbergi)

VK09

VK47

Hycleus dufourii (Graells, 1849)

Cercedilla (Trotta-Moreu \& García-París, 2001)

VL11

Cercedilla: El Ventorrillo, 1480m (Trotta-Moreu \& García-París, 2001) VL11

Cercedilla: Estación Alpina, 1500m (Trotta-Moreu \& García-París, 2001) VL11

El Escorial (Górriz Muñoz, 1882)

El Escorial (Trotta-Moreu \& García-París, 2001;

Pérez-Moreno et al., 2003)

VK09

UK99 
Guadarrama (Graells, 1849; De la Fuente, 1933)

Montejo de la Sierra (Trotta-Moreu \& García-París, 2001)

Montañas de Guadarrama (Graells, 1851a; Marseul, 1870)

Narichiva [Collado de Marichiva] (Graells, 1851a)

Navacerrada (Trotta-Moreu \& García-París, 2001)

Pinares de Navacerrada (Graells, 1851a)

Región montana media del Guadarrama (Graells, 1851b)

Hycleus duodecimpunctatus (Olivier, 1811)

Alberche (Trotta-Moreu \& García-París, 2001) [UTM Embalse de San Juan]

Alcalá (Trotta-Moreu \& García-París, 2001)

Alrededores de Madrid (Trotta-Moreu \& García-París, 2001)

[UTM Villaverde]

Aranjuez (Trotta-Moreu \& García-París, 2001)

El Escorial, 1200 m (Trotta-Moreu \& García-París, 2001)

Madrid: El Pardo (Trotta-Moreu \& García-París, 2001)

Madrid (Górriz Muñoz, 1882)

Madrid (Trotta-Moreu \& García-París, 2001)

Madrid, 689 m (Trotta-Moreu \& García-París, 2001)

Madrid: Moncloa (Trotta-Moreu \& García-París, 2001)

Montarco (Trotta-Moreu \& García-París, 2001)

Navacerrada (Trotta-Moreu \& García-París, 2001)

Madrid: Puerta de Hierro (Trotta-Moreu \& García-París, 2001)

Villaviciosa (Trotta-Moreu \& García-París, 2001)

Hycleus scutellatus (Rosenhauer, 1856)

Alrededores de Madrid (Trotta-Moreu \& García-París, 2001) Aranjuez (Trotta-Moreu \& García-París, 2001)

Carretera Vallecas-Montarco (Trotta-Moreu \& García-París, 2001)

Cercedilla (Trotta-Moreu \& García-París, 2001)

Cerro del Telégrafo, Rivas de Jarama (López-Colón, 2005)

El Escorial (Trotta-Moreu \& García-París, 2001; Pérez-Moreno et al., 2003)

El Escorial, 1200m (Trotta-Moreu \& García-París, 2001)

El Rincón (Trotta-Moreu \& García-París, 2001)

Madrid: Dehesa de la Villa (Trotta-Moreu \& García-París, 2001)

Madrid: El Pardo (Trotta-Moreu \& García-París, 2001)

Madrid: Getafe (Trotta-Moreu \& García-París, 2001)

Madrid: Madrid (Trotta-Moreu \& García-París, 2001)

Madrid: Moncloa (Trotta-Moreu \& García-París, 2001)

Madrid: Villaverde (Trotta-Moreu \& García-París, 2001)

Montarco [Rivas de Jarama] (Trotta-Moreu \& García-París, 2001)

Navacerrada (Trotta-Moreu \& García-París, 2001)

Ribas [Cristo de] (Trotta-Moreu \& García-París, 2001)

Rivas (Trotta-Moreu \& García-París, 2001)

Villaviciosa (Trotta-Moreu \& García-París, 2001)

Mylabris (Chalcabris) uhagonii Martínez Sáez, 1873

El Escorial (Martínez Sáez, 1873)

Madrid (Rodríguez López-Neyra, 1914)

Madrid (Sumakov, 1930)

Montareo [Montarco] (Sumakov, 1930)

Aranjuez (Górriz Muñoz, 1882)

Mylabris (Mesosulcata) hieracii Graells, 1849

Guadarrama (Graells, 1849; Marseul, 1870)

Montañas de Guadarrama (Graells, 1851a)

Subida de Navacerrada (Graells, 1851b)

Región media de Guadarrama (Graells, 1851b)

El Escorial (Seidlitz, 1867; Marseul, 1870; Górriz Muñoz, 1882)

El Escorial (Fuente, 1933, citado como M. h. var. suspiciosa)

El Escorial (Bologna, 1991; Pérez-Moreno et al., 2003)

La Pedriza (Pérez-Moreno et al., 2003)

Madrid (Pérez-Moreno et al., 2003)

Puerto de Somosierra (Pérez-Moreno et al., 2003)

Villaviciosa de Odón (Pérez-Moreno et al., 2003)

Mylabris (Micrabris) dejeani (Gyllenhal, 1817)

El Escorial (Pérez-Moreno et al., 2003)

Embalse de Navacerrada (Pérez-Moreno et al., 2003)

Embalse de Navacerrada, Collado Mediano (Pérez-Moreno et al., 2003) VL10

Madrid (Górriz Muñoz, 1882)
Mylabris (Micrabris) sobrina Graells, 1849

El Escorial (Pérez-Moreno et al., 2003)

El Ventorrillo - Sierra de Guadarrama (Pérez-Moreno et al., 2003)

Estación Alpina Cercedilla (Pérez-Moreno et al., 2003)

Guadarrama (Graells, 1849; De la Fuente, 1933)

Montañas de Guadarrama (Graells, 1851a; Marseul, 1870)

Región montana media de Guadarrama (Graells, 1851b)

El Escorial (Seidlitz, 1867; Górriz Muñoz, 1882)

Sierra de Guadarrama (Sumakov, 1930)

Mylabris (Micrabris) varians (Gyllenhal, 1817)

El Ventorrillo, Sierra de Guadarrama (Pérez-Moreno et al., 2003)

Puerto de Somosierra (Pérez-Moreno et al., 2003)

Mylabris (Mylabris) quadripunctata (Linnaeus, 1767)

Aranjuez (Górriz Muñoz, 1882)

Madrid (Amor Mayor, 1860; Górriz Muñoz, 1882)

Puerto de Somosierra (Pérez-Moreno et al., 2003)

Ventorrillo, Guadarrama (Pérez-Moreno et al., 2003)

KK09

VL11

VL10

VL00

VL00

VLO0

VK09

VL00

VL11

VL55

Mylabris (Mylabris) variabilis (Pallas, 1781)

El Escorial (Górriz Muñoz, 1882; Sumakov, 1930)

Puerto de Somosierra (Pérez-Moreno et al., 2003)

Carretera de Cercedilla a Navacerrada (Gómez Carrasco, 1888)

Madrid (Sumakov, 1930)

Euzonitis haroldi (Heyden, 1870)

Madrid (Rodríguez López-Neyra, 1914)

El Pardo (Rodríguez López-Neyra, 1914)

Casa de Campo, cerca de Madrid (Heyden, 1870)

$\mathrm{VK} 43$

VK47

VL55

VL01

VK09

VL55

VL10

VK47

Euzonitis quadrimaculata (Pallas, 1782)

Madrid (Rodríguez López-Neyra, 1914,

sub Z auricoma Escherich, 1891)

Aranjuez (Rodríguez López-Neyra, 1914

sub Z. a. var. bombycina Escherich, 1896)

VK47

VK43

Zonitis (Zonitis) flava Fabricius, 1775

Vaciamadrid (Pérez-Moreno et al., 2003)

VK58

Zonitis (Zonitis) immaculata (Olivier, 1789)

Madrid (Rodríguez López-Neyra, 1914)

Madrid (Pérez-Moreno et al., 2003)

Universidad Autónoma (Pérez-Moreno et al., 2003)

VK47

VK47

Apalus (Criolis) guerini (Mulsant, 1858)

Nuevo Baztán (García-París, 2000)

Sitaris (Sitaris) muralis (Foerster, 1771)

Alcorcón (Torres Sala, 1962, sub Apalus muralis)

Villaviciosa (García-París, 2000)

VK26

VK26

Stenoria (Stenoria) apicalis (Latreille, 1802)

El Escorial (García-París, 2000; Ruiz, 2001)

Cercedilla (Pardo Alcaide, 1958)

VK09 
Apéndice 2.- Observaciones de Meloidae en la Comunidad de Madrid (no necesariamente acompañadas de capturas; si no se indica el observador corresponden a M. García-París).

Appendix 2.- Field observations of Meloidae in Comunidad de Madrid (not all specimens observed were captured; if observer is not indicated the record was provided by M. García-París).

Cerocoma (Cerocoma) schaefferi (Linnaeus, 1758)

$3 \mathrm{~km}$ al S de San Martín de la Vega, $560 \mathrm{~m}: 14-\mathrm{VI}-1998,12$ ejemplares VK5049 $3 \mathrm{~km}$ E Pinilla del Valle: 22-VI-2000, 4 ejemplares

$5 \mathrm{~km}$ al S de Tielmes: 12-VI-2004, 18 ejemplares

$5 \mathrm{~km}$ al S de Tielmes: 4-VI-2004. 1 ejemplar

$5 \mathrm{~km}$ al S de Tielmes: 8-VII-2004, 1 ejemplar

$7 \mathrm{~km}$ al SE de Morata de Tajuña: 12-VI-2004, 40 ejemplares

Cerro Almodóvar, Vallecas, Madrid, $710 \mathrm{~m}$ : 11-VI-1998, 8 ejemplares

Cerro Almodóvar, Vallecas, Madrid, 710 m: 12-VI-1998, 4 ejemplares

Cerocoma (Metacerocoma) schreberi Fabricius, 1781

$2 \mathrm{~km}$ al S de Tielmes: 17-VI-2000, 13 ejemplares

$3 \mathrm{~km}$ al N de Villarejo de Salvanés: 7-VIII-2004, 5 ejemplares

$3 \mathrm{~km}$ al S de San Martín de la Vega, 560 m: 14-VI-1998, 1 ejemplar

$3 \mathrm{~km}$ al S de Tielmes: 15-VII-2000, 1 ejemplar

$3 \mathrm{~km}$ al S de Tielmes: 15 -VIII-2000, 2 ejemplares

$4 \mathrm{~km}$ al S de Tielmes: 30-VI-20025, 5 ejemplares

$5 \mathrm{~km}$ al N de Tielmes: 15-VII-2000, 2 ejemplares

$5 \mathrm{~km}$ al N de Villarejo de Salvanés: 7-VIII-2004, 3 ejemplares

$5 \mathrm{~km}$ al S de Tielmes: 12-VI-2004, 1 ejemplar

$5 \mathrm{~km}$ al S de Tielmes: 8-VII-2004, 1 ejemplar

$7 \mathrm{~km}$ al SE de Morata de Tajuña: 12-VI-2004, 8 ejemplare

Belmonte de Tajo - Encomienda Mayor de Castilla: 23-VI-2004, 1 ejempla

Cercedilla: 10-VII-1989, 1 ejemplar (F.A. Montes)

Berberomeloe majalis (Linnaeus, 1758)

$0.5 \mathrm{~km}$ al NE de Meco: 7-IV-1999, 1 ejemplar

$1 \mathrm{~km}$ al O de Brea de Tajo: 11-IV-1999, 1 ejemplar

$2 \mathrm{~km}$ al SE de Villanueva de la Torrre: 7-IV-1999, 1 ejemplar

$3 \mathrm{~km}$ al $\mathrm{N}$ de Canencia: 9-IV-1999, 1 ejemplar

$3 \mathrm{~km}$ al NE de Olmeda de las Fuentes, 820 m: 13-VI-1998, 8 ejemplares VK8371

$3 \mathrm{~km}$ al O de Valdilecha: 22-V-2004, 1 ejemplar

$3 \mathrm{~km}$ al SE de Valdelaguna: 5-V-2004, 1 ejemplar

$3 \mathrm{~km}$ al SE de Vicálvaro, $700 \mathrm{~m}: 28-\mathrm{IV}-2000,1$ ejemplar

$4 \mathrm{~km}$ al NE de Estremera: 07-V-2003, 2 ejemplares

$4 \mathrm{~km}$ al $\mathrm{O}$ de Valdelaguna: 12-VI-2005, 1 ejemplar

$5 \mathrm{~km}$ al O de Alcalá de Henares: 18-IV-1999, 2 ejemplares

Alcalá de Henares: II-1999, 1 ejemplar (G. Astudillo)

Caserío El Quexigal, Cebreros-Robledo de Chavela: 21-IV.

1 ejemplar (N. Trotta)

Madrid, Cerro Almodóvar, Santa Eugenia, 710 m: 20-V-1998, 1 ejemplar VK487

Madrid, Cerro Almodóvar, Vallecas, 710 m: 12-III-1989,

4 ejemplares (F.A. Montes)

VK4871

Madrid, Cerro Almodóvar, Vallecas, 710 m: 12-VI-1998, 2 ejemplares VK4871

Madrid, Cerro Almodóvar, Vallecas, 710 m: 23-III-1989, 1 ejemplar (F.A. Montes)

El Atazar: 23-IV-2005 4 ejemplares

Estremera, $6 \mathrm{~km}$ al NE de Belinchón, $600 \mathrm{~m}$ : 8-IV-2004, 1 ejemplar Estremera: $7 \mathrm{~km}$ al NE de Belinchón. $600 \mathrm{~m}$ : 8-IV-2004, 1 ejempla

Estremera: Cerro Casasola, $630 \mathrm{~m}$ : 8-IV-2004, 3 ejemplares

Mejorada del Campo: 24-V-2003, 1 ejemplar

Olmeda de las Fuentes: 16-VI-1997, 2 ejemplares

Valdilecha: 22-V-2004, 1 ejemplar

Lytta (Lytta) vesicatoria (Linnaeus, 1758)

$2 \mathrm{~km}$ al NO del Puerto de Somosierra: 6-VI-1999, 7 ejemplares

$3 \mathrm{~km}$ al E de Valdemanco: 28-V-2000, 12 ejemplares

La Barranca de Navacerrada: 5-VI-2000, 2 ejemplares

Laguna de Los Pájaros, 2100 m: 9-VII-1983, 2 ejemplares

Miraflores de la Sierra: 21-VI-1992, 4 ejemplares (F.A. Montes)

San Martín de Valdeiglesias, 760 m: 24-IV-2004, 8 ejemplares

VK4871

VK9138

VK9139

VK9140

VK5972

VK86

VK76

Oenas fusicornis Abeille de Perrin, 1880

$1 \mathrm{~km}$ al NO de Perales de Tajuña: 2-VI-2000, 35 ejemplares

VL45

VL42

VL11

VL1922

VL3

UK8268

VK65
$1 \mathrm{~km}$ al O de Valdilecha: 30-V-2000, 1 ejemplar

$2 \mathrm{~km}$ al E de Venturada, $800 \mathrm{~m}: 15-\mathrm{VI}-1985,1$ ejemplar VL4816

$2 \mathrm{~km}$ al S de Tielmes: 17-VI-2000, 1 ejemplar $\quad$ VK75

$3 \mathrm{~km}$ al S de Tielmes: 12-VI-2004, 20 ejemplares

$3 \mathrm{~km}$ al S de Tielmes: $3-\mathrm{VI}-2000$, más de 200 ejemplares

$5 \mathrm{~km}$ al S de Carabaña: 4-VI-2004, 13 ejemplares $\quad$ VK85

$5 \mathrm{~km}$ al S de Tielmes: 12-VI-2004, 1 ejemplar $\quad$ VK75

$5 \mathrm{~km}$ al S de Torrelaguna: 28-V-2003, $5 \quad$ VL51

$5 \mathrm{~km}$ al SO de Torrelaguna, $790 \mathrm{~m}$ : 13-VI-1998, más de 200 ejemplares VL5415

$6 \mathrm{~km}$ al NE de Villarejo de Salvanés: 4-VI-2004, 30 ejemplares VK84

Arroyo Vega, $1 \mathrm{~km}$ al O de Olmeda de las Fuentes, $780 \mathrm{~m}$ : 8-VII-1998, 5 ejemplares

Belmonte de Tajo: 23-VI-2005, 1 ejemplar $\quad$ VK74

El Espartal: 12-VI-1999, 2 ejemplares

VL51

Physomeloe corallifer (Germar, 1818)

$1 \mathrm{~km}$ al N de Canencia: 9-IV-1999, 1 ejemplar

VL32

$1 \mathrm{~km}$ al SO del Puerto de La Hiruela, 1300m: 24-IV-1999, 1 ejemplar VL54

$3 \mathrm{~km}$ al NO de Manzanares El Real, $900 \mathrm{~m}$ : 30-IV-2000, 1 ejemplar VL2409

$3 \mathrm{~km}$ al O de Villaviciosa de Odón, $650 \mathrm{~m}$ : 9-IV-2004, 1 ejemplar VK26

Cerro San Pedro: 24-V-2004, 3 ejemplares

Ciudad Universitaria, Madrid: 1-IV-1986, 3 ejemplares

Ciudad Universitaria, Madrid: 2-III-1983, 1 ejemplar

El Atazar: 23-IV-2005, 1 ejemplar

El Escorial: Fuente de la Teja: 4-IV-1999, 2 ejemplares

El Ventorrillo, Cercedilla: 22-III-1998, 1 ejemplar (F.A. Montes)

El Ventorrillo, Cercedilla: 28-II-1999, 7 ejemplares (F.A. Montes)

Embalse de Becerril, 1400 m: 5-III-2000, más de 100 ejemplares (F.A. Montes)

Las Herreras, Santa María de la Alameda: 23-III-2005, 1 ejemplar

Majadahonda: 09-III-2003, 1 ejemplar

Manjirón: 30-III-2002 (I. Martínez-Solano)

Monumento al Guarda Forestal, Rascafría, 1250 m: 9-III-2000,

13 ejemplares

VL30

VK3878

VK 3878

VL63

VK09

VL11

VL11

VL10

UK99

VK2481

VL5136

Navacerrada: Peña Pintada: 11-IV-1999, 1 ejemplar (F.A. Montes)

Presa del Atazar: 23-IV-2005, 1 ejemplar

Puerto de la Morcuera: 21-V-2004, 1 ejemplar

Puerto de La Morcuera: 8-III-1998, 1 ejemplar (F.A. Montes)

Puerto de Los Leones (Guadarrama): 8-III-1998, 1 ejemplar

(F.A. Montes)

Puerto de Malagón, Santa María de la Alameda: 3-V-1998,

1 ejemplar (F.A. Montes)

Puerto de Malagón: 4-IV-1999, 3 ejemplares

Zarzalejo: 22-III-2005, 1 ejemplar

VL2523

VL1512

VL62

VL32

VL32

VL00

UK99

VK09

Meloe (Coelomeloe) tuccius (Rossi, 1792)

Cerro Almodóvar, Vallecas, 710 m: 23-III-1989, 1 ejemplar (F.A. Montes) VK4871

Dehesa de la Villa, Madrid: 28-IV-1986, 1 ejemplar (F.A. Montes) VK3878

Presa del Atazar: 23-IV-2005, 18 ejemplares

Cerro del Viso, Torres de la Alameda: 25-IV-1999, 2 ejemplares

(G. Astudillo)

VK67

Meloe (Eurymeloe) mediterraneus G. Müller, 1925

$5 \mathrm{~km}$ al E de Colmenar Viejo: 30-X-2005, 1 ejemplar

Garganta de los Montes: 4-XI-2001, 1 ejemplar (I. Martínez-Solano)

VL30

Meloe (Eurymeloe) murinus Brandt et Erichson, 1832

$5 \mathrm{~km}$ al E de Colmenar Viejo: 30-X-2005, 1 ejemplar

Colmenar Viejo: 9-XI-1984, 1 ejemplar (F.A. Montes)

VL30

Meloe (Eurymeloe) nanus Lucas, 1849

$3 \mathrm{~km}$ al S de Tielmes: 3-XI-2000, 1 ejemplar

VK75

Meloe (Eurymeloe) rugosus Marsham, 1802 
Meloe (Meloe) proscarabaeus Linnaeus, 1758

$3 \mathrm{~km}$ al N de Miraflores de la Sierra, hacia el Pto. de Canencia: 9-IV-1999, 1 ejemplar

Puerto de Cotos: 8-III-1998, 1 ejemplar (F. A. Montes)

Hoya de Pepe Hernando : 12-IV-2002, 1 ejemplar

Actenodia billbergi (Gyllenhal, 1817)

$1 \mathrm{~km}$ al NE de Ciempozuelos, $560 \mathrm{~m}:$ 14-VI-1998, 1 ejemplar

$7 \mathrm{~km}$ al SE de Morata de Tajuña: 12-VI-2004, 1 ejemplar Cerro Almodóvar, Vallecas, 710 m: 11-VI-1998, 1 ejemplar

El Pardo: 29-V-1983, 1 ejemplar (F.A. Montes)

Villamanrique de Tajo: 23-VI-2004, 1 ejemplar

Hycleus dufourii (Graells, 1849)

Presa del Tobar: 14-VII-2005, 3 ejemplares

Santa María de la Alameda - El Tobar: 20-VII-2004, 4 ejemplares (I. Martínez-Solano)

Hycleus duodecimpunctatus (Olivier, 1811)

Arroyo Culebro, Perales del Río: 23-VI-2005, 1 ejemplar

Sierra de la Higuera: 21-VII-2004, 5 ejemplares

Hycleus scutellatus (Rosenhauer, 1856)

$1 \mathrm{~km}$ N Colmenar del Arroyo: 11-VI-2000, 7 ejemplares

$2 \mathrm{~km}$ al S de Navalagamella: 11-VI-2004, 15 ejemplares

$2 \mathrm{~km}$ al S de Tielmes: 17-VI-2000, 1 ejemplar

$3 \mathrm{~km}$ al S de Valdemorillo: 15-V-2005, 1 ejempla

$4 \mathrm{~km}$ al S de Tielmes: $30-\mathrm{VI}-2002,5$ ejemplares

Arroyo Culebro, Perales del Río: 23-VI-2005, 9 ejemplare

Belmonte de Tajo - Encomienda Mayor de Castilla: 23-VI-2004,

3 ejemplares

Cerro Telégrafo, 2 km SE del Cristo de Rivas, 640 m: 29-VI-1998, más de 50 ejemplares

Dehesa de Cepeda: 16-VI-2005, 5 ejemplares

El Campillo - Rivas: 5-VII-2004, 4 ejemplares

Las Infantas: 14-V-2005, 4 ejemplares

Puerto de la Cruz Verde: 22-VI-2005, 27 ejemplares

Robledo de Chavela: 21-VII-2004, 2 ejemplares

Santa María de la Alameda - El Tobar: 20-VII-2004, 1 ejemplar (I. Martínez-Solano)

Mylabris (Mesosulcata) hieracii Graells, 1849

$3 \mathrm{~km}$ al E de Valdemanco: 28-V-2000, 15 ejemplares

$3 \mathrm{~km}$ al S de Tielmes: 3-VI-2000, 1 ejemplar

$4 \mathrm{~km}$ al S de Robledo de Chavela, Pico Almenara, $850 \mathrm{~m}$ :

10-V-1998, 50 ejemplares

4 km NO Rascafría: 22-VI-2000 3 ejemplares

Becerril de la Sierra: 5-VII-2004, 1 ejemplar (R. Zardoya)

Cercedilla: 24-VII-1983, 1 ejemplar (F.A. Montes)

El Escorial: 4-V-1999, 1 ejemplar

El Pardo: 27-V-1987, 1 ejemplar (F.A. Montes)

La Barranca de Navacerrada: 5-VI-2000, 3 ejemplares

Puerto de Canencia: 22-VI-1999, 2 ejemplares

Puerto de Guadarrama: 14-VII-2005, 1 ejemplar

Puerto de Guadarrama: 18-VII-1998, 1 ejemplar

Puerto de la Morcuera: 21-VI-2003, 1 ejemplar

Puerto de los Cotos - Valdesquí: 17-VI-2004, 1 ejemplar

Rascafría, 1600 m: 7-VIII-2004, 1 ejemplar (R. López)

San Martín de Valdeiglesias, 760 m: 24-IV-2004, 1 ejemplar

Mylabris (Micrabris) maculosopunctata Graells, 1858

$1 \mathrm{~km}$ al NE de Ciempozuelos, $560 \mathrm{~m}:$ 14-VI-1998, 6 ejemplares

$2 \mathrm{~km}$ al SO de Colmenar de Oreja: 23-VI-2005, 1 ejemplar

$3 \mathrm{~km}$ al S de Quijorna: 11-VI-2004, 1 ejemplar

$3 \mathrm{~km}$ al S de Tielmes: 3-VI-2000, más de 200 ejemplares

$4 \mathrm{~km}$ al S de Tielmes: 30-VI-2002, 4 ejemplares

El Pardo: 29-V-1983, 3 ejemplares (F.A. Montes)

El Pardo: 1-VII-1984, 1 ejemplar (F.A. Montes)

Olmeda de Fuentes: 13-VI-2003, 1 ejemplar

Mylabris (Micrabris) sobrina Graells, 1849

3 km E Pinilla del Valle: 22-VI-2000,1 ejemplar

Calzada Romana, 1 km S del Puerto La Fuenfría: 26-VII-1998,

más de 200 ejemplares

Camino de la Laguna de los Pájaros: 22-VI-2000, 2 ejemplares
Laguna de Los Pájaros-Operante: 19-VII-2000, 4 ejemplares

(I. Martínez-Solano)

Laguna de Los Pájaros, 2100 m: 9-VII-1983

VL22

VL1922

VL0807

VL0405

VL11

Puerto de Guadarrama: 18-VII-1998, 8 ejemplares

Puerto de La Fuenfría: 26-VII-1998, 4 ejemplares

Puerto de la Morcuera: 21-VI-2003, 3 ejemplares

Puerto de los Cotos - Valdesquí: 17-VI-2004, 1 ejemplar

VK4845

Puerto de Malagón, 1600 m: 9-VII-1998, más de 50 ejemplare

VL32

VL11

VK0197

VL22

Valdesquí: 11-VI-2005, 4 ejemplares

VL11

Mylabris (Micrabris) varians (Gyllenhal, 1817)

3 km NE Meco: 20-VI-2000, 1 ejemplar

VK79

$4 \mathrm{~km}$ al NO de Nuevo Baztán, $820 \mathrm{~m} \cdot 8$-VII-1998, 6 ejemplares_ VK7871

$4 \mathrm{~km}$ NO Rascafría: 22-VI-2000, 1 ejemplar

$5 \mathrm{~km}$ al N de Tielmes: 15-VII-2000, 4 ejemplares

Cercedilla: 24-VII-1983, 1 ejemplar (F.A. Montes)

Puerto de Canencia, Río Canencia: 21-VIIL-1999, 1 ejemplar

Puerto de Guadarrama. 18-VII-1998, 7 ejemplares

VK46 Puerto de Guadarrama: 14-VII-2005, 10 ejemplares

UK65 Puerto de La Fuenfría: 26-VII-1998, 6 ejemplares

Puerto de Malagón, 1600 m: 9-VII-1998, 5 ejemplares

Santa María de la Alameda - El Tobar: 20-VII-2004, 3 ejemplares (I. Martínez-Solano)

VL23

VK75

VL11

VL32

VL0405

VL00

VL11

VK0197

UK99

UK97

VK07

VK75

VK08

VK75

VK46

VK73

Mylabris (Mylabris) quadripunctata (Linnaeus, 1767)

$1 \mathrm{~km}$ al NE de Ciempozuelos, $560 \mathrm{~m}$ : 14-VI-1998, 4 ejemplares VK4845 $1 \mathrm{~km} \mathrm{~S}$ del Hayedo de Montejo: 12-VI-2005, 1 ejemplar (A. Perdices) VL54

$2 \mathrm{~km}$ al S de Tielmes: 17-VI-2000, 4 ejemplares VK75

$2 \mathrm{~km}$ al SE de Paredes de Buitrago, $1050 \mathrm{~m}: 13-\mathrm{VII}-1998,6$ ejemplares VL5338

$2 \mathrm{~km}$ al SO de Colmenar de Oreja: 23-VI-2005, 6 ejemplares VK63

$2 \mathrm{~km}$ SO Arganda: 12-VI-2005, 1 ejemplar $\quad$ VK66

$3 \mathrm{~km}$ al E de Tielmes, hacia Carabaña: 17-VII-2000, 1 ejemplar VK75

$3 \mathrm{~km}$ al N de Lozoya: 6-VIL-2004, 2 ejemplares

$3 \mathrm{~km}$ al S de Tielmes: 12-VI-2004, 1 ejemplar $\quad$ VK75

$3 \mathrm{~km}$ al S de Tielmes: 15-VII-2000, 5 ejemplares $\quad$ VK75

$3 \mathrm{~km}$ al S de Tielmes: 15-VIII-2000, 3 ejemplares $\quad$ VK75

$3 \mathrm{~km}$ al S de Tielmes: 3-VI-2000 8 ejemplares

$3 \mathrm{~km}$ al SE de Villar del Olmo, $780 \mathrm{~m}:$ 13-VI-1998, 1 ejemplar $\quad$ VK8164

$3 \mathrm{~km}$ NE Meco: 20-VI-2000, 3 ejemplares $\quad$ VK79

$\begin{array}{ll}3 \mathrm{~km} \text { S Valdeavero: 20-VI-2000, } 1 \text { ejemplar } & \text { VK79 }\end{array}$

$3 \mathrm{~km}$ SO Navalagamella: 11-VI-2000, 2 ejemplares

4 km al NO de Nuevo Baztán, 820 m: 8-VII-1998, 16 ejemplares

VL42 $4 \mathrm{~km}$ al O de Morata de Tajuña, $700 \mathrm{~m}$ : 14-VI-1998, 1 ejemplar

$4 \mathrm{~km}$ al S de Tielmes: 30-VI-2002, 5 ejemplares

$4 \mathrm{~km}$ al SE de Arganda, $720 \mathrm{~m}$ : 30-VI-1998, 1 ejemplar

$5 \mathrm{~km}$ al $\mathrm{N}$ de Tielmes: 15-VII-2000, 3 ejemplares

UK9380

Belmonte de Tajo - Encomienda Mayor de Castilla: 23-VI-2004,

3 ejemplares

Cerro del Telégrafo, $2 \mathrm{~km}$ al SE del Cristo de Rivas, $640 \mathrm{~m}$ :

29-VI-1998, 4 ejemplares

El Atazar: 18-VIII-2000, 3 ejemplares

El Pardo: 27-V-1987, 1 ejemplar (F.A. Montes)

La Acebeda, 1200 m: 13-VII-1998, más de 50 ejemplares

Olmeda de las Fuentes: 15-VI-2003, 4 ejemplares

Puerto de Canencia: 22-VI-1999, 3 ejemplares

Puerto de Guadarrama: 14-VII-2005, 4 ejemplares

Puerto de Guadarrama: 18-VII-1998, 8 ejemplares

Puerto de la Cruz Verde: 22-VI-2005, 4 ejemplares

Puerto de la Fuenfría: 26-VII-1998, 1 ejemplar

Rascafría, 1600 m: 7-VIII-2004, 2 ejemplares (R. López)

Santa María de la Alameda - El Tobar: 20-VII-2004, 2 ejemplares

(I. Martínez-Solano)

K4845

VK63

VK17

VK75

VK 75

VK3486

VK86

Valdaracete: 15-VI-2003, 3 ejemplares

Valdetorres de Jarama: VII-1983, 3 ejemplares

Villamanrique de Tajo: 23-VI-2004, 1 ejemplar

VK07

VK7871

VK5652

VK75

VK6558

VK75

VK73

VK5679

VL63

VK3486

VL4747

VK86

VL32

VLO0

VL0405

UK99

VL11

VL22

UK99

VK85

VL5707

VK73

Mylabris (Mylabris) variabilis (Pallas, 1781)

$1 \mathrm{~km}$ al NO de Robledondo, 1400 m: 9-VII-1998, 2 ejemplares

UK9793

VL00

Puerto de Guadarrama: 14-VII-2005, 3 ejemplares

Valdetorres de Jarama: VII-1983, 4 ejemplares

Villamanrique de Tajo: 23-VI-2004, 5 ejemplares

VL5707

VK73

Euzonitis haroldi (Heyden, 1870)

VL11

$2 \mathrm{~km}$ al E de Venturada, $800 \mathrm{~m}$ : 15-VI-1985, 1 ejemplar

VL4816 
$4 \mathrm{~km}$ al SE de Arganda, $720 \mathrm{~m}:$ 9-VI-1998 1 ejemplar $5 \mathrm{~km}$ al S de Torrelaguna: 28-V-2003, 2 ejemplares

Euzonitis quadrimaculata (Pallas, 1782)

$2 \mathrm{~km}$ al E de Venturada, $800 \mathrm{~m}$ : 15-VI-1985, 2 ejemplares $3 \mathrm{~km}$ al E de Nuevo Baztán: 29-V-2003, 1 ejemplar $3 \mathrm{~km}$ al O de Valdilecha. $790 \mathrm{~m}: 10-\mathrm{VI}-1998,1$ ejemplar $3 \mathrm{~km}$ al S de Torrelaguna: $28-\mathrm{V}-2003,5$ ejemplares

$5 \mathrm{~km}$ al S de Tielmes: 4-VI-2004, 1 ejemplar

$5 \mathrm{~km}$ al S de Torrelaguna: $28-\mathrm{V}-2003,9$ ejemplares

$5 \mathrm{~km}$ al SO de Torrelaguna. $790 \mathrm{~m}$ : 13-VI-1998, 9 ejemplare

Torrelaguna-El Vellón: 10-VI-2000, 12 ejemplares

Euzonitis sexmaculata (Olivier, 1789)

Cercedilla: 15-VII-1986, 1 ejemplar (F.A. Montes)

Nemognatha (Nemognatha) chrysomelina (Fabricius, 1775)

$3 \mathrm{~km}$ al E de Tielmes, hacia Carabaña: 17-VII-2000, 1 ejemplar

$3 \mathrm{~km}$ al S de Tielmes: 15-VII-2000, 3 ejemplares

$3 \mathrm{~km}$ N de Villarejo de Salvanés; 7-VIII-2004, 2 ejemplares

Tielmes: 19-VII-2004, 1 ejempla

Villarejo de Salvanés: 19-VII-2004, 5 ejemplares
VK6558 Zonitis (Zonitis) flava Fabricius, 1775

VL5

$3 \mathrm{~km}$ al S de Tielmes: 15-VII-2000, 1 ejemplar

$3 \mathrm{~km}$ N de Villarejo de Salvanés: 7-VIII-2004, 1 ejemplar

$4 \mathrm{~km}$ al S de Tielmes: 30-VI-2002, 1 ejemplar

VL4816 $5 \mathrm{~km}$ al N de Tielmes: 15-VII-2000, 5 ejemplares

VK86 $5 \mathrm{~km} \mathrm{~S}$ de Tielmes: 8-VII-2004, 2 ejemplares

VK7160 Lozoya: 6-VII-1991, 3 ejemplares (F.A. Montes leg.)

Puerto de Guadarrama: 14-VII-2005, 1 ejemplar

Villarejo de Salvanés: 9-VII-2004, 4 ejemplares

VL 5

VL5415

VL5

Zonitis (Zonitis) immaculata (Olivier, 1789)

Olmeda de Fuentes: 15-VI-2003, 1 ejemplar

VK75

VK74

VK75

VK75

VK75

VL33

VL00

$\mathrm{VK} 74$

VK86

Sitaris (Sitaris) muralis (Foerster, 1771)

VL11 $4 \mathrm{~km}$ al S de Tielmes: 3-VIII-2004, 4 ejemplares (larvas) $4 \mathrm{~km}$ al $\mathrm{S}$ de Tielmes: 5-IX-2004, 5 ejemplares

Apéndice 3.- Datos de ejemplares de Meloidae de Madrid procedentes de colecciones científicas (ver texto para abreviaturas y acrónimos).

Appendix 3. - Data obtained from specimens of Meloidae of Madrid located in scientific collections (see text for abbreviations and acronyms).

Cerocoma (Cerocoma) schaefferi (Linnaeus, 1758)

Aravaca: 13-V-1984 (R. Sánchez leg.) (Col. RS)

Casa de Campo, Madrid: 21-V-1983 (R. Sánchez leg.) (Col. RS)

Casa de Campo, Madrid: 4-VI-1985 (R. Sánchez leg.) (Col. RS)

Cercanías de Camporreal: 27-V-1991 (R. Sánchez leg.) (Col. RS)

Cerro Almodóvar, Santa Eugenia: 15-V-1987 (R. Sánchez leg.) (Col. RS)

Cerro Grande de Almodóvar, Santa Eugenia: 7-V-1987 (R. Sánchez leg.) (Col. RS)

El Cuadrón: 25-VI-1994 (R. Sánchez leg.) (Col. RS)

El Pardo: 10-VI-1985 (R. Sánchez leg.) (Col. RS)

El Pardo: 16-VI-1987 (R. Sánchez leg.) (Col. RS

El Pardo: 26-V-1985 (R. Sánchez leg.) (Col. RS)

El Pardo: 27-V-1985 (R. Sánchez leg.) (Col. RS)

Embalse de El Pardo: 16-VI-1987 (R. Sánchez leg.) (Col. RS)

Embalse los Morales, Rozas de Puerto Real: 25-VI-1989 (R. Sánchez leg.) (Col. RS)

Moralzarzal: 9-VII-1982 (R. Sánchez leg.) (Col. RS)

Navacerrada: 3-VII-1988 (R. Sánchez leg.) (Col. RS)

Navacerrada: 5-VII-1987 (R. Sánchez leg.) (Col. RS)

Río Jarama, Mejorada del Campo: 12-VI-1983 (R. Sánchez leg.) (Col. RS)

Río Jarama, Mejorada del Campo: 24-V-1986 (R. Sánchez leg.) (Col. RS) VK57

Río Jarama, Mejorada del Campo: 31-V-1986 (R. Sánchez leg.) (Col. RS) VK57

San Sebastián de los Reyes: 19-VI-1988, 2 ejemplares

(J.L. Zapata leg.) (Col. JLZ)

Somonte, El Pardo: 29-V-1988 (R. Sánchez leg.) (Col. RS)

Villamanrique: 7-V-1964 (R. Sánchez leg.) (Col. RS)

Cerocoma (Metacerocoma) schreberi Fabricius, 1781

Arroyo Garguera, Guadalix de la Sierra: 25-VI-1994

(R. Sánchez leg.) (Col. RS)

VK37

VK37

VK37

VK27

VL43
VK38

VK38

VK73
Cercedilla: 4-VIII-1985 (R. Sánchez leg.) (Col. RS)

VL11

K66

VK38
VK38

VK38

VK38

VK38

UK66

VL10

VL10

VL10

VK57

VK57

VK48

$$
\text { (Col. RS) }
$$

E1 Pardo: 8-VII-1985 (R. Sánchez leg.) (Col. RS)

Embalse Valmayor: 13-VII-1989, 2 ejemplares (J.L. Zapata leg.) (Col. JLZ)

Garganta de Los Montes: 18-VII-1998 (R. Sánchez leg.) (Col. RS)

Garganta de Los Montes: 18-VII-2000 (R. Sánchez leg.) (Col. RS)

Guadarrama: 24-VI-1990, 2 ejemplares (J.L. Zapata leg.) (Col. JLZ)

Navacerrada: 5-VII-1987 (R. Sánchez leg.) (Col. RS)

Valdemorillo: 15-VIII-1963 (C. G. leg.) (Col. P. Oromí)

Valmayor: 13-VII-1989, 2 ejemplares (J.L. Zapata leg.) (Col. JLZ)

Berberomeloe majalis (Linnaeus, 1758)

Casa de Campo, Madrid: 20-IV-1984 (R. Sánchez leg.) (Col. RS)

Cerro Almodóvar: 14-IV-1989, 1 ejemplar (M.A.S. Sobrino leg.) (Col. MAS)

Cerro del Telégrafo, Rivas de Jarama: 11-IV-1987 (R. Sánchez leg.) (Col. RS)

Cerro del Telégrafo, Rivas de Jarama: 25-III-1988 (R. Sánchez leg.) (Col. RS)

Cerro Grande de Almodóvar, Vallecas: 15-IV-1987 (R. Sánchez leg.) (Col. RS)

Cerro Grande de Almodóvar, Vallecas: 15-V-1988 (R. Sánchez leg.) (Col. RS)

Cerro Grande de Almodóvar, Vallecas: 25-III-1988 (R. Sánchez leg.) (Col. RS)

VK27

VK38

VK18

VL43

VL43

VL00

VL10

VK08

VK18

Colmenar Viejo: 1-V-1985 (R. Sánchez leg.) (Col. RS

Corales, Rozas de Puerto Real: 9-V-1993

(R. Sánchez leg.) (Col. RS)

Griñón: 28-IV-1984, 3 ejemplares (P. Bercedo leg.) (Col. PBLA)

$\begin{array}{ll}\text { Guadarrama: 25-V-1986 (R. Sánchez leg.) (Col. RS) } & \text { VL00 } \\ \text { Los Almendros, Rivas de Jarama: 17-IV-1987 (R. Sánchez leg.) (Col. RS) } & \text { VK56 }\end{array}$
VK37

VK47

VK56

VK56

VK47

VK47

VK47

VL30

UK66

VK25

VK56 
Los Almendros, Rivas de Jarama: 17-V-1987 (R. Sánchez leg.) (Col. RS) VK56 Madrid: Dehesa de la Villa: 20-IV-1980, 1 ejemplar

(M.A.S. Sobrino leg.) (Col. MAS)

Madrid: Dehesa [de la Villa]: 27-III-1988, 1 ejemplar

(J.L. Zapata leg.) (Col. JLZ)

Madrid: Santa Eugenia: 27-V-1986, 1 ejemplar (P. Bercedo leg.) (Col. PBLA)

Matalpino [Mataelpino]: 20-III-1995, 1 ejemplar (J.L. Zapata leg.) (Col. JLZ)

Mejorada del Campo: 4-VI-1983 (R. Sánchez leg.) (Col. RS)

Río Jarama, Mejorada del Campo: 30-V-1987 (R. Sánchez leg

Vicálvaro: 6-VI-1986 (R. Sánchez leg.) (Col. RS)

Lytta (Lytta) vesicatoria (Linnaeus, 1758)

La Pedriza: 28-V-1989, 2 ejemplares (J.L. Zapata leg.) (Col. JLZ) Madrid: Madrid [Canillejas]: 13-VI-1984, 1 ejemplar

(P. Bercedo leg.) (Col. PBLA) Mirador, Miraflores de la Sierra: 26-VI-1992 (R. Sánchez leg.) (Col. RS) VL31 Subida al Puerto de Navafría, Lozoya: 28-V-1995 (R. Sánchez leg.)

(Col. RS)

Physomeloe corallifer (Germar, 1818)

Batres: 15-III-1987 (R. Sánchez leg.) (Col. RS)

Boadilla del Monte: 3-V-1986 (R. Sánchez leg.) (Col. RS)

Casa de Campo, Aravaca: 28-IV-1986 (R. Sánchez leg.) (Col. RS

Cercedilla: 1 ejemplar (Exp. del Museo) (Col. MZB)

Cercedilla: 1 ejemplar (Moróder leg.) (Col. MZB)

Cerro del Telégrafo, Rivas de Jarama: 11-IV-1987 (R. Sánchez leg.) (Col. RS)

Cerro Grande de Almodóvar, Vallecas: 7-III-1987 (R. Sánchez leg.) (Col. RS)

Cerro San Pedro: 21-II-1993, 1 ejemplar (J.L. Zapata leg.) (Col. JLZ) VL40

Chinchón: 10-V-1979, 1 ejemplar (M.A.S. Sobrino leg.) (Col. MAS) VK64 Cruce carretera Boadilla-Pozuelo: 5-V-1985 (R. Sánchez leg.) (Col. RS) VK27

Dehesa de la Villa: 16-V-1980, 1 ejemplar (M.A.S. Sobrino leg.) (Col. MAS)

Griñón: 10-V-1984, 1 ejemplar (P. Bercedo leg.) (Col. PBLA) Guadalix de la Sierra: 20-IV-1986 (R. Sánchez leg.) (Col. RS)

Guadarrama: 4-V-1986 (R. Sánchez leg.) (Col. RS)

La Dehesa [de la Villa]: 15-V-1988, 1 ejemplar (J.L. Zapata leg.) (Col. JLZ)

La Dehesa [de la Villa]: 27-III-1988, 1 ejemplar (J.L. Zapata leg.) (Col. JLZ)

Las Rozas: 11-IV-1959, 2 ejemplares (Gras leg.) (Col. MZB)

Loeches: 19-III-1987 (R. Sánchez leg.) (Col. RS)

Los Almendros, Rivas de Jarama: 19-III-1987 (R. Sánchez leg.) (Col. RS) VK56 Los Roncajales: 16-II-1996, 1 ejemplar (J.L. Zapata leg.) (Col. JLZ) VL30

Los Roncajales: 25-II-1996, 1 ejemplar (J.L. Zapata leg.) (Col. JLZ) VL30

Madrid: 16-V-1925, 1 ejemplar (Col. Español) (Col. MZB)

Madrid: 2 ejemplares (A. Sanz leg.) (Col. MZB)

Ventorrillo: VI-1946, 4 ejemplares (Mateu leg.) (Col. MZB)

Meloe (Coelomeloe) tuccius (Rossi, 1792)

Cerro Grande de Almodóvar, Vallecas: 27-IV-1977 (R. Sánchez leg.) (Col. RS)

Cerro Grande de Almodóvar, Vallecas: 20-IV-1987 (R. Sánchez leg.) (Col. RS)

Cerro Grande de Almodóvar, Vallecas: 25-III-1988 (R. Sánchez leg.) (Col. RS

Cerro Grande de Almodóvar, Vallecas: 28-III-1991 (R. Sánchez leg.) (Col. RS)

Madrid: 4 ejemplares (Col. MNCN)

Madrid: 1 ejemplar (Col. Pérez Arcas) (Col. MNCN)

Madrid: Casa de Campo: 1 ejemplar (Ceballos leg.) (Col. MNCN)

Madrid: Ciudad Universitaria: 21-III-43, 2 ejemplares (Col. MNCN)

Madrid: Dehesa de la Villa: 2 ejemplares (Peris Torres leg.) (Col. MNCN)

Madrid: Dominicos: 17-III-1987, 1 ejemplar (M. Candela leg.) (Col. MNCN)

Madrid: Puerta de Hierro: 21-IV-1945, 1 ejemplar (Peris leg.) (Col. MNCN)

Madrid: Virgen del Cortijo: 2-IV-77, 2 ejemplares (C. Rey leg.) (Col. MNCN)
Madrid: Virgen del Cortijo: 14-III-77, 1 ejemplar(C. Rey leg.) (Col. MNCN)

VK47

Madrid: Virgen del Cortijo: 9-III-77, 2 ejemplares (C. Rey leg.) (Col. MNCN)

adrid: El Pinar [de Chamartín]: 15-V-76, 1 ejemplar

(M. Candela leg.) (Col. MNCN)

Madrid: Alameda de Osuna: 1 ejemplar (Col. MNCN)

VK48

VK57

Madrid: Alameda de Osuna: V-1985, 1 ejemplar (Izquierdo leg.) (Col. MNCN)

VK57

Paracuellos de Jarama: VI-935, 1 ejemplar (B. Quirós leg.) (Col. MNCN) VK58

Meloe (Eurymeloe) brevicollis Panzer, 1793

Cerced. [Cercedilla]: 1 ejemplar (Col. MNCN)

Cercedilla: 1 ejemplar (J. Ardois leg.) (Col. MNCN)

Cercedilla: $15-\mathrm{V}-1826,1$ ejemplar (Col. MNCN)

Cercedilla: 3 ejemplares (Col. MNCN)

Cercedilla: 3 ejemplares (G. Schramm leg.) (Col. MNCN)

Cercedilla: 4 ejemplares (Exp. del Museo) (Col. MNCN)

Cercedilla: 4 ejemplares (J. Lauffer leg.) (Col. MNCN)

Cercedilla: 8 ejemplares (C. Bolívar leg.) (Col. MNCN)

Cercedilla: V-1898, 1 ejemplar

El Escorial, Guadarrama:1 ejemplar (G. Schramm leg.) (Col. MNCN)

El Escorial: 2 ejemplares (Arias leg.) (Col. MNCN)

El Escorial: 2 ejemplares (Bolívar leg.) (Col. MNCN)

El Escorial: 2 ejemplares (C. Bolívar leg.) (Col. MNCN)

El Escorial: 2 ejemplares (Col. MNCN)

El Escorial: 2 ejemplares (J. Lauffer leg.) (Col. MNCN)

El Paular: X-1908, 1 ejemplar (Bolívar leg.) (Col. MNCN)

Madrid: 1 ejemplar (Barras leg.) (Col. MNCN)

Madrid: 1 ejemplar (Col. MNCN)

Madrid: 2 ejemplares (Col. del Sr. Pérez Arcas) (Col. MNCN)

2 ejemplares (C. Rodríguez leg.) (Col. MNCN) VL54

Navacerr. [Navacerrada]: 30-IV-1899, 4 ejemplares (Col. MNCN) VL10

Puerto de los Leones: 1-V-1987,1 ejemplar (J.L. Zapata leg.) (Col. JLZ) VL00

Puerto de Navafría: 23-IV-2004 (R. Sánchez leg.) (Col. RS) VL33

Puerto de Navafría: 8-V-2005 (R. Sánchez leg.) (Col. RS) VL33

Puerto de Somosierra: 1-V-1992 (R. Sánchez leg.) (Col. RS) VL55

Sierra de Guadarrama: 1 ejemplar (Col. MNCN) VL00

Sierra de Guadarrama:2 ejemplares (J. Lauffer leg.) (Col. MNCN) VL00

Somosierra; 14-VI, 1 ejemplar (Col. MNCN)

Villaviciosa: 1 ejemplar (J. Ardois leg.) (Col. MNCN)

VK26

Meloe (Eurymeloe) mediterraneus G. Müller, 1925

Cienvallejos, Brunete: 1 ejemplar (C. Bolívar leg.) (Col. MNCN)

El Escorial: 1 ejemplar (Col. Pérez Arcas) (Col. MNCN)

El Escorial: 1 ejemplar (M.A. Bologna det. 1992) (Col. MNCN)

El Escorial: 2 ejemplares (Col. MNCN)

Madrid: 1 ejemplar (Escribano leg.) (Museo) (Col. MNCN)

Madrid: 1 ejemplar (M.A. Bologna det. 1992) (Col. Pérez Arcas) (Col. MNCN)

Madrid: 4 ejemplares (Col. MNCN)

Madrid: 6 ejemplares (J. Ardois leg.) (Col. MNCN)

Madrid: Dehesa de la Villa: 16-V-1980, 1 ejemplar

(M.A.S. Sobrino leg.) (Col. MAS)

Móstoles: 1 ejemplar (J. Ardois leg.) (Col. MNCN)

VK17

VK09

VK09

VK09

VK47

VK47

VK47

VK47

VK37

VK26

Meloe (Eurymeloe) murinus Brandt et Erichson, 1832

Alcalá de Henares; 1 ejemplar (M. A. Bologna det.1992) (Col. MNCN) VK68 Madrid: 1 ejemplar (Col. MNCN)

Meloe (Eurymeloe) nanus Lucas, 1849

Aranjuez: 1 ejemplar (M.A. Bologna det. 1992) (Col. Pérez Arcas) (Col. MNCN)

Montarco [Rivas del Jarama]:1 ejemplar (M.A. Bologna det. 1992) (Col. MNCN)

VK56

Meloe (Lampromeloe) cavensis Petagna, 1819

«V. Vall.» [Vía Vallecas]: 1 ejemplar (Col. MNCN)

Aranjuez: 1 ejemplar (F. Arias leg.) (Col. MNCN)

VK47

Madrid: 1 ejemplar (Col del Sr. Pérez Arcas) (Col. MNCN) VK47

Madrid: 4 ejemplares (Col. MNCN)

Madrid: 5 ejemplares (C. Bolívar leg.) (Col. MNCN)

Vallecas: 1 ejemplar (G. Carrasco leg.) (Col. MNCN) 
Meloe (Lampromeloe) variegatus Donovan, 1793

Madrid: 1 ejemplar (Col. MNCN)

Perales de T. [Tajuña]: 1 ejemplar (Hidalgo leg.) (Col. Pérez Arcas) (Col. MNCN)

Rozas de Puerto Real: 1-V-1904, 1 ejemplar (M. A. Bologna det., 1992) (Col. MNCN)

Meloe (Meloe) proscarabaeus Linnaeus, 1758

Cercedilla: 1 ejemplar (Bolívar leg.) (Col. MNCN) Guadalix de la Sierra: 2-V-1986 (R. Sánchez leg.) (Col. RS) Madrid: 1 ejemplar (Col. MNCN)

Madrid: Tranv.Vall.[Tranvía Vallecas], XII-97 [1897], 1 ejemplar (Col. MNCN)

Paracuellos del Jarama: VI-935, 1 ejemplar (B. Quirós leg.) (Col. MNCN)

Perales [de Tajuña]: 1 ejemplar (Hidalgo leg.) (Col. MNCN) Pinto: 1 ejemplar (Col. MNCN)

Puerto de la Fuenfría: 4-IV-1999 (R. Sánchez leg.) (Col. RS) Rivas [de Jarama]: 1 ejemplar (Dusmet leg.) (Col. MNCN) Villarejo [de Salvanés]: 1 ejemplar (Col. MNCN)

Meloe (Meloe) violaceus Marsham, 1802

Cercedilla: 24-IV-98 [1898], 1 ejemplar (Col. MNCN) Cercedilla:1 ejemplar (Moróder leg.) (Col. MNCN) Cercedilla: V-98 [1898], 1 ejemplar (Col. MNCN)

Cercedilla: 1 ejemplar (G. Mercet leg.) (Col. MNCN) Cercedilla: V-1909, 1 ejemplar (Bolívar leg.) (Col. MNCN) Cercedilla: 1 ejemplar (Museo) (Col. MNCN)

El Escorial: 1 ejemplar (C. Bolívar leg.) (Col. MNCN)

El Escorial: 1 ejemplar (J. Ardois leg.) (Col. MNCN)

Navacerrada: 1 ejemplar (Col. del Sr. Pérez Arcas) (Col. MNCN)

Somosierra: 14-VI, 1 ejemplar (Col. MNCN)

Meloe (Treiodus) autumnalis Olivier, 1792

Cercedilla: 1 ejemplar (C. Bolívar leg.) (Col. MNCN)

Cercedilla: 4 ejemplares (Museo) (Col. MNCN)

Cercedilla: IV-1921, 1 ejemplar (Col. MNCN)

El Escorial: 8-XI-1915,1 ejemplar (Col. MNCN)

El Paular: 1 ejemplar (Arias leg.) (Col. MNCN)

El Paular: 1 ejemplar (Col. MNCN)

Madrid: 1 ejemplar (J. Ardois leg.) (Col. MNCN)

Matalpino: 20-III-1995, 1 ejemplar (J.L. Zapata leg.) (Col. JLZ)

Peñalara: 1 ejemplar (C. Bolívar leg.) (Col. MNCN)

Vía Vallecas: 15-XI- 96 [1896], 1 ejemplar (Col. MNCN)

Actenodia billbergi (Gyllenhal, 1817)

Colmenar Viejo: 18-V-1990, 1 ejemplar (M.A.S. Sobrino leg.) (Col. MAS)

Hycleus duodecimpunctatus (Olivier, 1811)

Arroyo Antequina, Casa de Campo: 11-VII-1987 (R. Sánchez leg.) (Col. RS)

Casa de Campo: 9-VII-1984 (R. Sánchez leg.) (Col. RS)

Casa de Campo: 7-VII-1985 (R. Sánchez leg.) (Col. RS)

Cerro Alarcón, Valdemorillo: 22-VII-1984 (R. Sánchez leg.) (Col. RS)

Cruce carretera Boadilla- Pozuelo: 14-VII-1985 (R. Sánchez leg.)

(Col. RS)

Puente de los Franceses, Casa de Campo: 6-VII-1985 (R. Sánchez leg.) (Col. RS)

Puente de los Franceses, Casa de Campo: 16-VI-1986 (R. Sánchez leg.) (Col. RS)

Villaviciosa de Odón: 1-VIII-1984 (R. Sánchez leg.) (Col. RS)

Hycleus scutellatus (Rosenhauer, 1856)

Cerro Alarcón, Valdemorillo: 4-VII-1987 (R. Sánchez leg.) (Col. RS) Cruce carretera Boadilla- Pozuelo: 5-VII-1985 (R. Sánchez leg.) (Col. RS)

El Pardo: 7-VI-1986 (R. Sánchez leg.) (Col. RS)

El Pardo, embalse: 16-VI-1987 (R. Sánchez leg.) (Col. RS

Puente Río Guadarrama, Villaviciosa: 11-VII-1987 (R. Sánchez leg.) Col. RS)

Mylabris (Chalcabris) uhagonii Martínez Sáez, 1873

Aranjuez: 30-VI-1907, 2 ejemplares (C. Rodríguez leg.) (Col. MNCN) El Escorial: 1 ejemplar (G. Carrasco leg.) (Col. MNCN)
El Escorial: 1 ejemplar (J. Lauffer leg.) (Col. MNC N)

El Escorial:1 ejemplar (Col. MNCN)

El Pardo: 1 ejemplar (Arias leg.) (Col. MNCN)

El Pardo: 1 ejemplar (J. Lauffer leg.) (Col. MNCN)

Getafe: 1 ejemplar (S.V. Peris leg.) (Col. MNCN)

Madrid: 1 ejemplar (A. Sainz leg.) (Col. MNCN)

Madrid: 2 ejemplares (J. Ardois leg.) (Col. MNCN)

Madrid: 33 ejemplares (J. Abajo leg.) (Col. MNCN)

Madrid: 8 ejemplares (Col. MNCN)

VL11 Madrid: Casa de Campo: VI-1908, 4 ejemplares (Bolívar leg.)

$$
\text { (Col. MNCN) }
$$

Madrid: Salón del Prado: 1 ejemplar (Col. MNCN)

Montarco: 12 ejemplares (G. Schramm leg.) (Col. MNCN)

Montarco: 2 ejemplares (Col. MNCN)

Montarco: 4 ejemplares (Bolívar leg.) (Col. MNCN)

K5 58

VK65

VL1

VK5

VK74

Montarco:

6, 2 ejemplares (Col. MNCN)

Ribas: 15 ejemplares (Bolívar leg.) (Col. MNCN)

Rivas: 1 ejemplar (Bolívar leg.) (Col. MNCN)

Rivas: 3-VII-1908 1 ejemplar (C. Rodríguez leg.) (Col. MNCN)

Rivas: 4 ejemplares (G. Carrasco leg.) (Col. MNCN)

Valdemoro: 2 ejemplares (F. Beltrán leg.) (Col. MNCN)

VL1

VL1

VL11

VK09

VL11

VL55

(Dusmet leg.) (Col. MNCN)

Mylabris (Mesosulcata) hieracii Graells, 1849

Alberche: VI-1906, 4 ejemplares (Arias leg.) (Col. MNCN)

Alberche: VI-1906, 6 ejemplares (Arias leg.)

(Pardo Alcaide det.) (Col. MNCN)

Alcobendas: 1 ejemplar (Col. MNCN)

Alpedrete: 6-V-1983 (R. Sánchez leg.) (Col. RS)

Cercanías de Miraflores, hacia La Morcuera: 12-VI-1990

(R. Sánchez leg.) (Col. RS)

VK09

VK09

VK38

VK38

VK38

VK47

VK47

VK47

VK47

VK37

VK47

VK56

VK56

VK56

VK56

VK56

VK56

VK57

VK57

VK57

VK57

K44

VK26

LII

VL11

VL11

VK09

VL22

VL22

K4 7

VL20

VL12

VK46

Cercedilla: 1 ejemplar (C. Bolívar leg.) (Pardo Alcaide det.

Cercedilla: 1 ejemplar (J. Lauffer leg.) (Col. MNCN)

Cercedilla: 1 ejemplar (Pardo Alcaide det.) (Col. MNCN)

Cercedilla: 2 ejemplares (F. Bonet leg.) (Col. MNCN)

Cercedilla: 24-VII-1893, 1 ejemplar (Col. MNCN)

Cercedilla: 25 ejemplares (Moróder leg.) (Col. MNCN)

Cercedilla: 2-VII-1898, 3 ejemplares (Col. MNCN)

Cercedilla: 5 ejemplares (Exp. del Museo) (Col. MNCN)

Cercedilla: 6 ejemplares (Museo) (Col. MNCN)

Cercedilla: 8 ejemplares (C. Bolívar leg.) (Col. MNCN)

Cercedilla: Estación Alpina, 1460 m: VII-1956, 4 ejemplares

(J. Abajo leg.) (Col. MNCN)

Cercedilla: Estación Alpina, 1500 m: VII-1935, 3 ejemplares

(J. Hernández leg.) (MNCN)

UK87

UK87

VK46

VL10

VL31

VL30

Estacion Alpina: 3 ejemplares (D. Peláez leg.) (Col.

Cojer (Col MAS)

Colmenar Viejo: 1-V-1985 (R. Sánchez leg.) (Col. RS)

VK37

VK37

VK37

(Col. RS)

5 (R. Sánchez leg.) (Col. RS)

Cruce carret

(Col. RS)

VL11

VL11

VL11

VL11

VL11

VL11

VL11

VL11

VL11

VL11

VL11

(J.L. Zapata leg.) (Col. JLZ)

El Escorial (G. Carrasco) (Col. Escherich)

El Escorial, Fuente de la Teja: 1 ejemplar (Col. MNCN)

El Escorial, pinar: 4 ejemplares (Col. MNCN)

El Escorial, pinares: 1 ejemplar (J. Ardois leg.) (Col. MNCN)

El Escorial, pinares: 11 ejemplares (Lauffer leg.) (Col. MNCN)

El Escorial, Puerto: 1 ejemplar (Graells leg.) (Col. MNCN)

VK08 El Escorial: 1 ejemplar (Arias leg.) (Pardo Alcaide det.) (Col. MNCN)

El Escorial: 1 ejemplar (Col. Pérez Arcas) (Col. MNCN)

VL11

El Escorial: 1 ejemplar (J. Abajo leg.) (Pardo Alcaide det) (Col. MNCN) VK09

VK38 El Escorial: 1 ejemplar (J. Abajo leg.) (Col. MNCN) VK09

$\begin{array}{lll}\text { VK38 } & \text { El Escorial: 1 ejemplar (J. Abajo leg.) (Col. MNCN) } & \text { VK09 } \\ \text { VK38 } & \text { El Escorial: } 11 \text { ejemplares (Col. MNCN) } & \text { VK09 }\end{array}$

$\begin{array}{ll}\text { El Escorial: } 11 \text { ejemplares (Col. MNCN) } & \text { VK09 } \\ \text { El Escorial: 16-VI-1907, } 1 \text { ejemplar (Pardo alcaide det.) (Col. MNCN) } & \text { VK09 }\end{array}$

El Escorial: 2 ejemplares (Arias leg.) (Col. MNCN) VK09

El Escorial: 2-V, 1 ejemplar (Pardo Alcaide det.) (Col. MNCN)

El Escorial: 5 ejemplares (G. Carrasco leg.) (Col. MNCN)

VK09

E1 Escorial: 8 ejemplares (Lauffer leg.) (Col. MNCN)
VK09

VK09

VL30

VL30

VK27

VK27

K27

VK09

VK09

VK09

VK09

K99

09

ro9
$\mathbf{1} 09$

VK09 
El Pardo: 1 ejemplar (C. Rodríguez leg.) (Pardo Alcaide det.) (Col. MNCN)

El Pardo: 12-V-1956, 1 ejemplar (S.V. Peris leg.) (Pardo Alcaide det.) (Col. MNCN)

El Pardo: 12-VII, 2 ejemplares (C. R. leg.) (Col. MNCN)

El Pardo: 14-V-1957, 1 ejemplar (J. Templado leg.) (Pardo Alcaide det.) (Col. MNCN)

El Pardo: 21-IV-1907, 3 ejemplares (Graells leg.) (Col. MNCN)

El Pardo: 21-VII-1908, 3 ejemplares (C. Rodríguez leg.) (Col. MNCN) El Pardo: 22 ejemplares (Arias leg.) (Col. MNCN)

El Pardo: 27-V-1987, 5 ejemplares (F.A. Montes leg.) (Col. MNCN)

El Pardo: 4 ejemplares (Arias leg.) (Pardo Alcaide det.) (Col. MNCN)

El Pardo: 4 ejemplares (J. Lauffer leg.) (Col. MNCN)

El Pardo: 4-VI-1907, 4 ejemplares (C. Rodríguez leg.) (Pardo Alcaide det.) (Col. MNCN)

El Pardo: 4-VII-1956, 1 ejemplar (J. Templado leg.) (Pardo Alcaide det.) (Col. MNCN)

El Pardo: 6 ejemplares (Col. MNCN)

El Pardo: 61 ejemplares (Bolívar leg.) (Col. $\mathrm{MNCN})$

El Pardo: V-1896, 3 ejemplares (Col. MNCN)

El Pardo: VI-1908, 2 ejemplares (Arias leg.) (Col. MNCN)

El Pardo: VI-1908, 3 ejemplares (Arias leg.) (Pardo Alcaide det.) (Col. MNCN)

El Pardo: X-1908, 1 ejemplar (Arias leg.) (Pardo Alcaide det.) (Col. MNCN)

El Rincón: 9 ejemplares (Col. MNCN)

Embalse de Valmayor: 2-VII-1990, 1 ejemplar (J.L. Zapata leg.) (Col. JLZ)

Madrid: 1 ejemplar (G. Carrasco leg.) (Graells det.) (Col. MNCN)

Madrid: 1 ejemplar (Graells leg.) (Col. MNCN)

Madrid: 1 ejemplar (M. Escalera leg.) (Col. MNCN)

Madrid: 1 ejemplar (Pardo Alcaide det.) (Col. MNCN)

drid: 2 ejemplares (C. Bolivar leg.) (Pardo Alcaide det.) (Col. MNCN) VK47

Madrid: 2 ejemplares (E. Morales leg.) (Col. MNCN)

Madrid: 4 ejemplares (C. Bolívar leg.) (Col. MNCN)

Madrid: 4 ejemplares (Col. MNCN)

Madrid: 45 ejemplares (A. Sainz leg.) (Col. MNCN)

VK38

VK38

VK38

VK38

VK38

VK38

VK3

VK3

VK38

VK38

VK38

VK38

VK38

VK38

VK38

VK38

VK3

UK96

VK18

VK47

VK4

VK47

VK47

VK47

VK47

VK47

VK47

Madrid: 5 ejemplares (J. Ardois leg.) (Pardo Alcaide det.) (Col. MNCN) VK47

Madrid 6 ejemplares (A. Sainz leg) (Pardo Alcaide det) (Col. MNCN) VK47

Madrid: 74 ejemplares (J. Ardois leg.) (Col. MNCN)

Madrid: Casa de Campo: 11-VI-1956, 1 ejemplar (J. Álvarez leg.) (P. Alcaide d.) (MNCN)

Madrid: Casa de Campo: VI-1972, 1 ejemplar (V. Llorente leg.) (Col. MNCN)

Madrid: Dehesa de la Villa: 9-VII-1909, 1 ejemplar (C. R. leg.) (Col. MNCN)

Madrid: Fuencarral; 8 ejemplares (G. Schramm leg.) (Col. MNCN) VK4

Madrid: Moncloa: 19-VII-1907, 2 ejemplares (Graells leg.) (Col. MNCN) VK37

Madrid: Moncloa: 26-VI-1907, 2 ejemplares (Graells leg.) (Col. MNCN) VK37

Madrid: Moncloa: 4-VII-1908, 1 ejemplar (C. Rodríguez leg.) (Col. MNCN)

Madrid: Moncloa: 8-VII-1908, 1 ejemplar (C. R. leg.) (Col. MNCN) VK37

Madrid: V-1961, 1 ejemplar (Col. MNCN)

Montejo de la Sierra: 2 ejemplares (C. Bolívar leg.) (Col. MNCN)

Móstoles: 6 ejemplares (J. Ardois leg.) (Pardo Alcaide det.) (Col. MNCN) VK26

Navacerrada: 1 ejemplar (J. Ardois leg.) (Col. MNCN)

Navacerrada: 2 ejemplares (Col. MNCN)

Navacerrada: 2 ejemplares (Uhagón leg.) (Pardo Alcaide det.) (Col. MNCN) VL10

Navalquejigo, 15-V-1957, 21 ejemplares (E. Ortiz leg.) (Pardo Alcaide det.) (Col. MNCN)

Navalquejigo: 17-V-1957, 13 ejemplares (J. Álvarez leg.) (Pardo Alcaide det.) (Col. MNCN)

VK19

VK19

Puerto de Canencia: 2-VI-1988, 1 ejemplar (J.L. Zapata leg.) (Col. JLZ) VL32

Puerto de Somosierra: 5-V11-1995, 1 ejemplar (P. Bahillo leg.)

(Col. MNCN)

VL55

San Agustín de Guadalix: 18-V-1982, 1 ejemplar (F.M. Piera leg.) VL40

San Sebastián de los Reyes: 25-VI-1988, 1 ejemplar

(J.L. Zapata leg.) (Col. JLZ)

Somontes, El Pardo: 29-V-1988 (R. Sánchez leg.) (Col. RS)

Soto del Real: 6-VI-1985, 1 ejemplar (C. Viejo leg.) (Col. MNCN)

Valdemorillo: 6-VI-1985 (R. Sánchez leg.) (Col. RS)

Villalba: 18-VII-1960, 1 ejemplar (Col. MNCN)

Villaviciosa de Odón: 19-V-1987 (R. Sánchez leg.) (Col. RS)

Villaviciosa: 20-6-1922, 1 ejemplar (Col. MNCN)
Mylabris (Micrabris) dejeani (Gyllenhal, 1817)

Cercedilla: 1 ejemplar (Moróder leg.) (Col. MNCN)

VL11

El Cuadrón, Garganta de los Montes: 27-VI-1993 (R. Sánchez leg.)

Col. RS)

El Escorial: 1 ejemplar (Escribano leg.) (Col. MNCN)

El Escorial: 2 ejemplares (Col. MNCN)

El Escorial: 43 ejemplares (Lauffer leg.) (Col. MNCN)

El Escorial: V, 47 ejemplares (F. Escalera leg.) (Col. MNCN)

El Escorial: VI, 2 ejemplares (F. Escalera leg.) (Col. MNCN)

Madrid: 1 ejemplar (J. Lauffer leg.) (Col. MNCN)

Madrid: 2 ejemplares (Mercet leg.) (Col. MNCN)

Madrid: 4 ejemplares (Mercet leg.) (Col. MNCN)

Madrid: 8 ejemplares (Col. MNCN)

Madrid: Canal: 1 ejemplar (Graells) (Col. MNCN)

Madrid: Canal: 20-VII-1897, 1 ejemplar (Col. MNCN)

Madrid: Canal: 25-VI-1899, 1 ejemplar (Col. MNCN)

Madrid: Canal: 3 ejemplares (Col. MNCN)

Madrid: Canal: 5-IX-1897, 2 ejemplares (Col. MNCN)

Madrid: Casa de Campo: 31-V-53, 7 ejemplares (E. Ortiz leg.)

(P. Alcaide det.) (MNCN)

VK56

Navacerrada: 25-VII-1897, 1 ejemplar (Col. MNCN) VL10

Navacerrada: 29-1897, 1 ejemplar (Col. MNCN) VL10

Rascafría: Puerto de la Morcuera, 1 ejemplar (L. Bágena leg.) (Col. $\mathrm{MNCN})$

Villaverde: 18- VII-1908, 20 ejemplares (C. Rodríguez leg.) (Col. MNCN)

Villaverde: 2 ejemplares (Col. MNCN)

VK36
-

Villaverde: 23- VI-1907, 4 ejemplares (Col. MNCN)

Mylabris (Micrabris) maculosopunctata Graells, 1858

Alberche: 1 ejemplar (Mercet leg.) (Col. MNCN)

Aranjuez: 2-VI- 1907, 1 ejemplar (Col. MNCN)

Casa de Campo, Madrid: 9-VI-1984 (R. Sánchez leg.) (Col. RS)

Cerro Alarcón, Valdemorillo: 4-VII-1987 (R. Sánchez leg.) (Col. RS)

Chinchón: 1 ejemplar (Col. Peréz Arcas) (Pardo Alcaide det.)

(Col. MNCN)

Chinchón: 12 ejemplares (Col. MNCN)

Chinchón: 2 ejemplares (Valera leg.) (Col. MNCN)

Ciempozuelos: 1 ejemplar (Col. Peréz Arcas) (Pardo Alcaide det.) (Col. MNCN)

El Escorial, Urb. los Arroyos: 15-VI-1980, 1 ejemplar (C. Rey leg.) (Col. MNCN)

El Escorial: 1 ejemplar (Lauffer leg.) (Col. MNCN)

El Escorial: 1 ejemplar (Zarco leg.) (Col. MNCN)

El Escorial: 3 ejemplares (Col. MNCN)

El Escorial: V, 55 ejemplares (F. Escalera leg.) (Col. MNCN)

El Pardo: 22-V-1985 (R. Sánchez leg.) (Col. RS)

El Pardo, Embalse: 8-VII-1985 (R. Sánchez leg.) (Col. RS)

El Pardo, Lomas del Corcho: 20-V-1989 (R. Sánchez leg.) (Col. RS)

El Pardo, Mingorrubio: 12-VI-1987 (R. Sánchez leg.) (Col. RS)

El Pardo: 1 ejemplar (Arias leg.) (Col. MNCN)

El Pardo: 29-V-1983, 1 ejemplar (M. García París leg.) (Col. MNCN) VK38

El Rincón : V-1907, 7 ejemplares (Col. MNCN) UK96

Guadarrama: VII-1956, 1 ejemplar (L. Báguena leg.) (Col. MNCN) VL00

Leganés: La Fortuna: V-1928,1 ejemplar (J. Dusmet leg.) (Col. MNCN) VK36

Madrid: Moncloa: 13 ejemplares (Col. MNCN) $\quad$ VK37

Montarco: 1 ejemplar (C. Bolívar leg.) (Col. MNCN) VK56

Montarco: 1 ejemplar (Dusmet leg.) (Col. MNCN)

Montarco: 1 ejemplar (G. Ceballos leg.) (Col. MNCN) VK56

Montarco: 1 ejemplar(F. Bonet leg.) (Col. MNCN) VK56

Montarco: 10-VI-1919, 3 ejemplares (Col. MNCN) VK56

Montarco: 2 ejemplares (Arias leg.) (Col. MNCN)

Montarco: 23-V-1918, 2 ejemplares (Col. MNCN)

Montarco: 4-VI-1924, 1 ejemplar (Col. MNCN) VK56

Montarco: 55 ejemplares (Lauffer leg.) (Col. MNCN) VK56

Montarco: 6 ejemplares (Col. MNCN) VK56

Montarco: 9 ejemplares (G. Schramm leg.) (Col. MNCN) VK56

Montarco: IV-1916, 5 ejemplares (Col. MNCN) VK56

Montarco: V-1918, 17 ejemplares (Col. MNCN) VK56

Montarco: VI-1908, 24 ejemplares (Arias leg.) (Col. MNCN) VK56

Montarco: VI-1916, 4 ejemplares (Col. MNCN) $\quad$ VK56

Navacerrada: 2-V-1897, 1 ejemplar (Col. MNCN) VL10

Paracuellos de Jarama: 3 ejemplares (J. Dusmet leg.) (Col. MNCN) VK58 
Ribas: 10 ejemplares (Bolívar leg ) (Pardo Alcaide det.) (Col. MNCN) VK57 Ribas: 2 ejemplares (Col. MNCN)

VK57

Río Jarama, Mejorada del Campo: 9-VI-1984 (R. Sánchez leg.) (Col. RS) VK57

Rivas: 2 ejemplares (Bolívar leg.) (Col. MNCN)

Rivas: 4 ejemplares (Bolivar leg) (Pardo Alcaide det) (Col. MNCN) VK57

Torrelaguna: 9 ejemplares (F. Beltrán leg.) (Pardo Alcaide det.)

(Col. MNCN)

Mylabris (Micrabris) sobrina Graells, 1849

Cercedilla: 1 ejemplar (Bolívar leg.) (Col. MNCN)

Cercedilla: 1 ejemplar (Pardo Alcaide det.) (Col. MNCN)

Cercedilla: 2 ejemplares (Museo) (Col. MNCN)

Cercedilla: 24 ejemplares (Moróder leg.) (Pardo Alcaide det.) (Col. MNCN)

Cercedilla: 4 ejemplares (Col. MNCN)

Cercedilla: 47 ejemplares (Moróder leg.) (Col. MNCN) VL11

Cercedilla: 9 ejemplares (Museo) (Pardo Alcaide det.) (Col. MNCN) VL11

Cercedilla: El Ventorrillo: 1480m, 4 ejemplares (J. Abajo leg.) (Col. MNCN)

Cercedilla: Estación Alpina: 1500m: VIII-1935, 3 ejemplares

(J. Hernández leg.) (MNCN)

El Escorial, Puerto: 8 ejemplares (Col. MNCN)

El Escorial: 1 ejemplar (Graells leg.) (Col. MNCN)

El Escorial: 11 ejemplares (Col. MNCN)

El Escorial: 17 ejemplares (Lauffer leg.) (Col. MNCN)

El Escorial: 3 ejemplares (Graells leg.) (Col. MNCN)

El Escorial: 42 ejemplares (Bolívar leg.) (Col. MNCN)

El Escorial: 6 ejemplares (Bolívar leg.) (Pardo Alcaide det.) (Col. MNCN)

El Escorial: 6 ejemplares (Escribano leg.) (Col. MNCN)

El Escorial: VI-1907, 3 ejemplares (Graells leg.) (Col. MNCN)

El Escorial: VII-1908, 1 ejemplar (Arias leg.) (Col. MNCN)

El Palancar, Morcuera, Km. 17, Rascafría : 12-VII-1992

(R. Sánchez leg.) (Col. RS)

Escurial [El Escorial]. (D. Seidlitz) (Col. Escherich)

Guadarrama: 1 ejemplar (Pardo Alcaide det.) (Col. MNCN)

Los Molinos: 23-VII-1985, 1 ejemplar (Col. MNCN)

Lozoya: 1 ejemplar (F. Navarro leg.) (Col. MNCN)

(Col. MNCN) VL33

Montejo de la Sierra: 4 ejemplares (Pardo Alcaide det.) (Col. MNCN) VL54

Montejo de la Sierra: 6 ejemplares (C. Bolívar leg.) (Col. MNCN) VL54

Montejo de la Sierra: 9-VI-1907, 1 ejemplar (Graells leg.) (Col. MNCN) VL54

Montejo de la Sierra: 9-VI-1907, 2 ejemplares (Graells leg.)

(Col. MNCN)

Navacerrada: 1 ejemplar (Col. MNCN)

Navacerrada: 10-VII-1953, 1 ejemplar (Á. Gauceiro leg.) (P. Alcaide det) (Col. MNCN)

Navacerrada: 2 ejemplares (J. Ardois leg.) (Col. MNCN)

Navacerrada: 20-VII-1909, 2 ejemplares (Arias leg.) (Col. MNCN)

Navacerrada: 20-VII-1953,2 ejemplares (A. Gauceiro leg.) (P. Alcaide det)

(Col. MNCN)

Navacerrada: 21-VII-1953, 2 ejemplares (W. Steiner leg.) (Pardo Alcaide det.)

(Col. MNCN)

Navacerrada: 25- VII- 1897, 5 ejemplares (Graells leg.) (Col. MNCN) VL10

Navacerrada: 7-VII-1953, 8 ejemplares (W. Steiner leg.)

(Pardo Alcaide det.) (Col. MNCN)

Navacerrada: VI, 1 ejemplar (J. Sanz leg.) (Col. MNCN)

Puerto de Guadarrama: 27-VII-1985, 2 ejemplares (P. Bercedo leg.) (Col. PBLA)

Puerto de la Morcuera: 21-VI-1992 (R. Sánchez leg.) (Col. RS)

Puerto de la Morcuera: 2-VII-1994 (R. Sánchez leg.) (Col. RS

Puerto de la Morcuera: 3-VII-1998 (R. Sánchez leg.) (Col. RS)

Puerto de Los Cotos: 8-VI-1990, 1 ejemplar (M.A.S. Sobrino leg.) (Col. MAS

Puerto de Navafría: 16-VII-2000 (R. Sánchez leg.) (Col. RS

Puerto de Navafría: 18-VII-2000 (R. Sánchez leg.) (Col. RS)

Puerto de Navafría: 28-VII-2000 (R. Sánchez leg.) (Col. RS)

Somosierra: 2 ejemplares (Col. MNCN)

Mylabris (Micrabris) varians (Gyllenhal, 1817)

Cerced.[illa]: 1 ejemplar (Col. MNCN)

Cercedilla: 2 ejemplares (Col. MNCN)

Cercedilla: 25-VII-1926, 1 ejemplar (Col. MNCN)

Cercedilla: 3 ejemplares (Moróder leg.) (Pardo Alcaide det., 1947)

(Col. MNCN)
Cercedilla: 4-VIII-1908, 1 ejemplar (C. Rodríguez leg.) (P. Alcaide det.) Col. MNCN)

VL11

Cercedilla: VIII-[18]96, 2 ejemplares (Col. MNCN) VL11

Cruce carretera Boadilla- Pozuelo: 6-VII-1985 (R. Sánchez leg.) (Col. RS) VK27

El Cuadrón, Garganta de los Montes: 27-VI-1993 (R. Sánchez leg.) (Col. RS)

El Escorial: 1 ejemplar (Carrasco leg.) (Pardo Alcaide det., 1947) (Col. MNCN)

El Escorial: 1 ejemplar (Col. Pérez Arcas) (Pardo Alcaide det.) (Col. MNCN)

El Escorial: 1 ejemplar (J. Lauffer leg.) (Col. MNCN)

El Escorial: 4 ejemplares (Col. MNCN)

El Escorial: 7 ejemplares (Lauffer leg.) (Col. MNCN)

El Escorial: Puerto [de Malagón]: 1 ejemplar (Col. MNCN)

El Escorial: Puerto [de Malagón]: 7 ejemplares (Lauffer leg.) (Col. MNCN)

El Escorial: V, 103 ejemplares (F. Escalera leg.) (Col. MNCN)

El Escorial: VI, 3 ejemplares (F. Escalera leg.) (Col. MNCN)

El Escorial: VII, 1 ejemplar (F. Escalera leg.) (Col. MNCN)

El Palancar, Morcuera, Km. 17, Rascafría : 12-VII-1992

(R. Sánchez leg.) (Col. RS)

El Palancar, Morcuera, Km. 17, Rascafría : 5-VII-1992 (R. Sánchez leg.) (Col. RS)

VK09

VK09

VK09

VK09

VK09

UK99

UK99

VK09

VK09

VK09

VL22

VL22

Km. 29, hacia Morcuera, desde Rascafría : 22-VI-1998

(R. Sánchez leg.) (Col. RS)

VL22

$\begin{array}{ll}\text { Montarco [Rivas de Jarama]: 1-VII-1917, } 1 \text { ejemplar (Col. MNCN) } & \text { VK56 } \\ \text { Montarco [Rivas de Jarama]: 30-V-[18]97, } 1 \text { ejemplar (Col. MNCN) } & \text { VK56 }\end{array}$

Montarco [Rivas de Jarama]: 30-V-[18]97, 1 ejemplar (Col. MNCN) VK56

Montarco [Rivas de Jarama]: 5 ejemplares (Lauffer leg.) (Col. MNCN) VK56

Montarco [Rivas de Jarama]: VI-1916, 1 ejemplar (Col. MNCN)

: 1 ejemplar (Col. Pérez Arcas) (Pardo Alcaide det., 1947) (Col. MNCN)

Navacerrada : 26-VII-[18]97, 1 ejemplar (Col. MNCN)

Puerto de Guadarrama: 27-VII-1985, 1 ejemplar (P. Bercedo leg.)

(Col. PBLA)

Puerto de Navafría: 16-VII-2000 (R. Sánchez leg.) (Col. RS)

Puerto de Navafría: 28-VII-2000 (R. Sánchez leg.) (Col. RS)

VL10

VL00

VL33

VL33

Mylabris (Mylabris) amorii Graells, 1858

El Rincón: V-1909, 135 ejemplares (Col. MNCN)

UK96

Mylabris (Mylabris) quadripunctata (Linnaeus, 1767)

Alcalá de Henares: VI-1950, 1 ejemplar (A. Chaves leg.) (Col. MNCN) VK68 Alcobendas: 1 ejemplar (Col. MNCN)

Alrededores de Madrid: VII-1959, 1 ejemplar (UTM Cerro de los Angeles) (Col. MNCN)

Aranjuez: 30-VI-1907, 4 ejemplares (C. Rodríguez leg.) (Col. MNCN) VK43

Aranjuez: 30-VI-1907, 7 ejemplares (Col. MNCN) VK43

Brunete: 1 ejemplar (Col. MNCN)

Campo Real: 25-VI-1987, 1 ejemplar (V. Llorente leg.) (Col. MNCN)

Casa de Campo: 28-VII-1984 (R. Sánchez leg.) (Col. RS) VK37

Casa de Campo: 30-V-1985 (R. Sánchez leg.) (Col. RS) VK37

Casa de Campo: 30-VII-1984 (R. Sánchez leg.) (Col. RS) VK37

Casa de Campo: 6-VII-1985 (R. Sánchez leg.) (Col. RS)

Cercedilla: 1 ejemplar (F. Bonet leg.) (Col. MNCN) VL11

Cercedilla: 17-VII-1957, 1 ejemplar (S.V. Peris leg.) (Col. MNCN) VL11

Cercedilla: 2 ejemplares (Peris Torres leg.) (Col. MNCN) VL11

Cercedilla: 22 ejemplares (Col. MNCN) VL11

VL10 Cercedilla: 4 ejemplares (C. Bolívar leg.) (Col. MNCN) VL11

VL10 Cercedilla: 5 ejemplares (Moróder leg.) (Col. MNCN) VL11

$\begin{array}{ll}\text { Cercedilla: 6-VII-1985 (R. Sánchez leg.) (Col. RS) } & \text { VL11 }\end{array}$

VL00 Cercedilla: Estación Alpina, 1460m: VII-1956, 17 ejemplares

(J. Abajo leg.) (MNCN)

Cercedilla: Estación Alpina, 1500m: VIII-1936, 1 ejemplar

(J. Hernández leg.) (MNCN)

VL11

Cercedilla: Estación Alpina: 4 ejemplares (D. Peláez leg.) (Col. MNCN) VL11

Cercedilla: IV-1896, 1 ejemplar (Col. MNCN) VL11

Cerro Alarcón, Valdemorillo: 22-VII-1984 (R. Sánchez leg.) (Col. RS) VK08

Colmenar Viejo: 15-VI-1990, 1 ejemplar (S. Sobrino leg.) (Col. MAS) VL30

Cruce carretera Boadilla- Pozuelo: 17-VII-1985 (R. Sánchez leg.) (Col. RS)

Cruce carretera Boadilla- Pozuelo: 30-VI-1984 (R. Sánchez leg.) (Col RS)

VK27

VK27

Cruce carretera Boadilla- Pozuelo: 5-VII-1985 (R. Sánchez leg.) (Col. RS)

Dehesa el Sotillo, Villaviciosa de Odón: 21-VI-1987 (R. Sánchez leg.) Col. RS)

VK27

Escorial, Fuente de la Teja: 2 ejemplares (Col. MNCN) 
El Escorial: 1 ejemplar (Escribano leg.) (Col. MNCN)

El Escorial: 6 ejemplares (Lauffer leg) (Col. MNCN)

El Escorial: 9 ejemplares (Col. MNCN) VK09

El Pardo: 21-VII-1908, 2 ejemplares (C. Rodríguez leg.) (Col. MNCN) VK38

El Pardo: 23-V-1986 (R. Sánchez leg.) (Col. RS) VK38

El Pardo: 27-V-1987, 2 ejemplares (F. A. Montes leg.) (Col. MNCN) VK38

El Pardo: 8-VII-1985 (R. Sánchez leg.) (Col. RS)

El Paular: X-1909, 1 ejemplar (Arias leg.) (Col. MNCN)

El Rincón: 14 ejemplares (Col. MNCN)

El Rincón: V-1909, 1 ejemplar (Col. MNCN)

Guadarrama: 1 ejemplar (Col. MNCN)

Guadarrama: VII-1956, 4 ejemplares (L. Báguena leg.) (Col. MNCN)

Km. 29, hacia Morcuera, desde Rascafría : 22-VI-1998

(R. Sánchez leg.) (Col. RS)

Loeches: 1 ejemplar (G. Carrasco leg.) (Col. MNCN)

Madrid: 1 ejemplar (C. Bolívar leg.) (Col. MNCN)

Madrid: 2 ejemplares (G. Carrasco leg.) (Col. MNCN)

Madrid: 21-VII-1960, 1 ejemplar (Col. MNCN)

Madrid: 3 ejemplares (Arias leg.) (Col. MNCN)

Madrid: 5-VII-1960, 1 ejemplar (Col. MNCN)

Madrid: 6 ejemplares (A. Sanz leg.) (Col. MNCN)

Madrid: 6 ejemplares (Col. MNCN)

Madrid: 9 ejemplares (J. Ardois leg.) (Col. MNCN)

Madrid: Dehesa de la Villa: 23-VII-1907, 1 ejemplar (P. Bahillo leg.) (Col. MNCN)

Madrid: Fuencarral: 1 ejemplar (G. Schramm leg.) (Col. MNCN)

Madrid: Moncloa: 1 ejemplar (G. Schramm leg.) (Col. MNCN)

Madrid: Moncloa: 2 ejemplares (Col. MNCN)

Madrid: Moncloa: 26-VII-1907, 1 ejemplar (Col. MNCN)

Madrid: Moncloa: 8-VIII-1908, 3 ejemplares (C. Rodríguez leg.) (Col. MNCN)

Madrid: V-1961, 3 ejemplares (Col. MNCN)

Montarco: 1 ejemplar (F. Bonet leg.) (Col. MNCN)

Montarco: 2 ejemplares (C. Bolívar leg.) (Col. MNCN)

Montarco: 2 ejemplares (Col. MNCN)

Montarco: 20-VII-1914, 1 ej (Lauffer leg.) (Col. MNCN)

Montarco: 30-V-1897, 1 ejemplar (Col. MNCN)

Montarco: 47 ejemplares (Arias leg.) (Col. MNCN)

Montarco: 5 ejemplares (G. Schramm leg.) (Col. MNCN)

Montarco: IV-1896, 1 ejemplar (Col. MNCN)

Montarco: VI-1908, 1 ejemplar (Arias leg.) (Col. MNCN)

Montarco: VII-1951, 2 ejemplares (Col. MNCN)

Montejo de la Sierra: 2 ejemplares (M. Escalera leg.) (Col. MNCN)

Montejo de la Sierra: 9-VII-1907, 2 ejemplares

(C. Rodríguez leg.) (Col. MNCN)

Navacerrada: 1 ejemplar (Arias leg.) (Col. MNCN)

Navacerrada: 25-VII-1897, 1 ejemplar (Col. MNCN)

Puente Río Guadarrama, Villaviciosa: 11-VII-1987

(R. Sánchez leg.) (Col. RS)

Puente Río Guadarrama, Villaviciosa: 4-VII-1992 (R. Sánchez leg.) (Col. RS)

Ribas: 1 ejemplar (Dusmet leg.) (Col. MNCN)

Ribas: 4 ejemplares (Bolívar leg.) (Col. MNCN)

Río Jarama, Mejorada del Campo: 31-V-1986 (R. Sánchez leg.) (Col. RS) VK57

Río Jarama, San Fernando-Mejorada Km7.7: 30-V-1987

(R. Sánchez leg.) (Col. RS)

Torrelodones: 20-IX-1962, 1 ejemplar (Col. MNCN)

Torres de la Alameda: 16-VII-1999, 2 ejemplares (J.L. Zapata leg.)

(Col. JLZ)

Valdetorres de Jarama: VII-1983, 4 ejemplares (M. García-París leg.)

(Col. MNCN)

Vicálvaro: 30-VI-1907, 1 ejemplar (C. Rodríguez leg.) (Col. MNCN)

Villalba: 1 ejemplar (Col. MNCN)

Villanueva (P.R.): 1918, 2 ejemplares (Smith leg.)

[UTM V. del Pardillo] (Col. MNCN)

Villanueva (P.R.): V-1919, 2 ejemplares (Smith leg.)

[UTM V. del Pardillo] (Col. MNCN)

Villaverde: 18-VII-1908, 6 ejemplares (C. Rodríguez leg.) (Col. MNCN) VK36

Villaverde: 23-VII-1907, 3 ejemplares (C. Rodríguez leg.) (Col. MNCN) VK36

Villaverde: 3 ejemplares (A. Sanz leg.) (Col. MNCN)

Villaverde: 5 ejemplares (G. Schramm leg.) (Col. MNCN)

Villaviciosa de Odón: 10-VI-1987 (R. Sánchez leg.) (Col. RS)

Villaviciosa: 3 ejemplares (J. Ardois leg.) (Col. MNCN)

Mylabris (Mylabris) variabilis (Pallas, 1781)

Río Jarama, Mejorada del Campo: 16-VI-1981 (R. Sánchez leg.)
(Col. RS)

VK57

Alcobendas: 1 ejemplar (Col. MNCN) $\quad$ VK48

Aranjuez: 1 ejemplar (G. Carrasco leg.) (Col. MNCN) VK43

Aranjuez: 30-VI-1907, 1 ejemplar (C. Rodríguez leg.) (Col. MNCN) VK43

Cercedilla: 2 ejemplares (C. Bolívar leg.) (Col. MNCN)

Cercedilla: 2 ejemplares (J. Lauffer leg.) (Col. MNCN) VL11

Cercedilla: 5 ejemplares (Moróder leg.) (Col. MNCN) VL11

Cercedilla: 6-VIII-1945, 8 ejemplares (Peris Torres leg.) (Col. MNCN) VL11

Cercedilla: Est. Alpina, 1460m: 1/10-VIII-1960, 1 ejemplar

(J. Abajo leg.) (Col. MNCN)

Cercedilla: Est. Alpina, 1500m: VIII-1935, 2 ejemplares

(J. Hernández leg.) (Col. MNCN)

VL11

Cercedilla: Estación Alpina: 1 ejemplar (D. Peláez leg.) (Col. MNCN) VL11

Cruce carretera Boadilla- Pozuelo: 17-VII-1985 (R. Sánchez leg.)

(Col. RS)

El Escorial, Puerto: 7 ejemplares (Lauffer leg) (Col. MNCN)

El Escorial: 16-VI-1907, 3 ejemplares (C. Rodríguez leg.) (Col. MNCN) VK09

El Escorial: 18-VI-1907, 2 ejemplares (C. Rodríguez leg.) (Col. MNCN) VK09

El Escorial: 20 ejemplares (Lauffer leg.) (Col. MNCN) VK09

El Escorial: 21-VII-1957, 1 ejemplar (A. García Velázquez leg.)

(Col. MNCN) VK09

El Escorial: 28-VII-1907, 3 ejemplares (C. Rodríguez leg.) (Col. MNCN) VK09

El Escorial: 6 ejemplares (Col. MNCN) VK09

El Pardo: 21-VII-1908, 1 ejemplar (C. Rodríguez leg.) (Col. MNCN) VK38

El Ventorrillo, Sierra Guadarrama: VII-1957, 5 ejemplares

(F. Español leg.) (Col. MZB)

Guadarrama: 1 ejemplar (Closes leg.) (Col. MZB)

Guadarrama: VI-1896, 1 ejemplar (Col. MNCN)

Guadarrama: VII-1956, 3 ejemplares (L. Báguena leg.) (Col. MNCN)

Madrid: 1 ejemplar (Arias leg.) (Col. MNCN)

Madrid: 1 ejemplar (Bolívar leg.) (Col. MNCN)

Madrid: 1 ejemplar (G. Carrasco leg.) (Col. MNCN)

Madrid: 14 ejemplares (A. Sanz leg.) (Col. MNCN)

Madrid: 6 ejemplares (Col. MNCN)

Madrid: 6 ejemplares (J. Ardois leg.) (Col. MNCN)

Madrid: Canal: 1 ejemplar (Col. MNCN)

Madrid: Canal: 15-VII-1897, 1 ejemplar (Col. MNCN)

Madrid: Casa de Campo: 1 ejemplar (Col. MNCN)

Madrid: Casa de Campo: 25-VI-1953, 1 ejemplar (E. Ortiz leg.) (Col. MNCN)

Madrid: Dehesa de la Villa: 2-VII-1909, 3 ejemplares (C. Rodríguez leg.) (Col. MNCN)

Madrid: Dehesa de la Villa: 8-VII-1909, 1 ejemplar

(C. Rodríguez leg.) (Col. MNCN)

Madrid: Dehesa de la Villa: 9-VII-1909, 5 ejemplare

(C. Rodríguez leg.) (Col. MNCN)

Madrid: Moncloa: 14-VII-1908, 3 ejemplares (C. Rodríguez leg.) (Col. MNCN)

Madrid: Moncloa: 19-VII-1907, 6 ejemplares (C. Rodríguez leg.) (Col. MNCN)

Madrid: Moncloa: 19-VII-1908, 2 ejemplares (C. Rodríguez leg.) (Col. MNCN)

Madrid: Moncloa: 26-VI-1907, 1 ejemplar (C. Rodríguez leg.) (Col. MNCN)

Madrid: Moncloa: 27-VII-1907, 1 ejemplar (C. Rodríguez leg.) (Col. MNCN)

Madrid: Moncloa: 8-VIII-1908, 1 ejemplar (C. Rodríguez leg.) (Col. MNCN)

Madrid: V-1961, 1 ejemplar (Col. MNCN)

Montarco: 1 ejemplar (Lauffer leg.) (Col. MNCN)

Montarco: VI-1917, 1 ejemplar (Col. MNCN)

Puerto de Navacerrada: VII-1957, 4 ejemplares (F. Español leg.) (Col. MZB)

Puerto de Somosierra: 5-VII-1985, 1 ejemplar (P. Bahillo leg.) (Col. MNCN)

VL11

VL00

VL00

VK47

VK47

VK47

VK47

VK47

VK47

VK47

VK47

VK37

VK37

VK37

VK37

VK37

VK37

VK37

VK37

VK37

VK37

VK37

VK47

VK56

VK56

VL11

VL55

VL00

Torres de la Alameda: 16-VIL-1999, 1 ejet leg.) (Col. MNCN) (Col. JLZ)

Valdetorres de Jarama: VII-1983, 6 ejemplares (M. García-París leg.) (Col. MNCN)

VK67

Vicálvaro: 30-VII-1907, 2 ejemplares (C. Rodríguez leg.) (Col. MNCN) VK57

Villalba: 1 ejemplar (Col. MNCN) VK19

Villaverde: 18-VII-1908, 10 ejemplares (C. Rodríguez leg.) (Col. MNCN) VK36

Villaverde: 2 ejemplares (Col. MNCN) VK36

Villaverde: 20-VI, 1 ejemplar (Col. MNCN) $\quad$ VK36

Villaverde: 23-VII-1907, 11 ejemplares (C. Rodríguez leg.) (Col. MNCN) VK36

Villaverde: 27-VII-1907, 2 ejemplares (C. Rodríguez leg.) (Col. MNCN) VK36 
Villaverde: 3 ejemplares (G. Schramm leg.) (Col. MNCN) Villaverde: 31-VII-1896, 3 ejemplares (Col. MNCN) Villaviciosa: 1 ejemplar (J. Ardois leg.) (Col. MNCN)

Euzonitis haroldi (Heyden, 1870)

M. Loeches: 1 ejemplar (G. Carrasco leg.) (M. A. Bologna det., 1992) (Col. MNCN)

Madrid: 1 ejemplar (J. Ardois leg.) (Col. MNCN)

Madrid: 1 ejemplar (M. A. Bologna det., 1992) (Col. MNCN)

Euzonitis quadrimaculata (Pallas, 1782)

Aranjuez: 12 ejemplares (G. Mercet leg.) (Col. MNCN)

Aranjuez: 30-VI-1907, 1 ejemplar (Col. MNCN)

Ciudad Universitaria: 22-V-1957, 1 ejemplar (E. Ortiz leg.)

(P.Alcaide det.) (Col. MNCN)

El Pardo: 1 ejemplar (Col. MNCN)

El Pardo: 1 ejemplar (J. Ardois leg.) (Col. MNCN)

El Pardo: 23 ejemplares (Arias leg.) (Col. MNCN)

El Pardo: 7 ejemplares (Bolívar leg.) (Col. MNCN)

El Pardo: VI-1921, 1 ejemplar (Col. MNCN)

El Pardo: 1 ejemplar (Col. MNCN)

Madrid: 1 ejemplar, Dr. Martin, Slg. R. Oberthür, 1956 (Kaszab det.) (Col. Kaszab)

Madrid: 1 ejemplar (G. Carrasco leg.) (Col. MNCN)

Madrid: 2 ejemplares (Col. MNCN)

Madrid: 3 ejemplares (A. Sanz leg.) (Col. MNCN)

Madrid: 74 ejemplares (J. Ardois leg.) (Col. MNCN)

Madrid: Santa Eugenia: 27-V-1986, 1 ejemplar (P. Bercedo leg.) (Col. PBLA)

Nuevo Baztán: 15-VI-1927, 1 ejemplar (Col. MNCN)

Nuevo Baztán: 15-VI-1928, 1 ejemplar (Col. MNCN)

Villaviciosa: 20-VI-1927, 6 ejemplares (Col. MNCN)

Euzonitis sexmaculata (Olivier, 1789)

Madrid: 1 ejemplar (Col. Pérez Arcas) (Col. MNCN)

Villaverde: VII-1896, 1 ejemplar (Col. MNCN)

Nemognatha (Nemognatha) chrysomelina (Fabricius, 1775)

Ribas, 1 ejemplar (G. Mercet leg.) (Col. MNCN)
VK36

Arroyo Antequina, Casa de Campo: 11-VII-1987 (R. Sánchez leg.) (Col. RS)

Navacerrada: 5-VII-1987 (R. Sánchez leg.) (Col. RS)

Casa de Campo: 10-VI-1985 (R. Sánchez leg.) (Col. RS)

El Pardo: 21-VII-1908, 1 ejemplar (C. Rodríguez leg.) (Col. MNCN) VK38

El Paular: 1 ejemplar (Gelphe leg.) (Col. MNCN) VL22

VK47 Madrid: 1 ejemplar (Col. MNCN) VK47

VK47 Madrid: 1 ejemplar (Col. del Sr. Pérez Arcas) (Col. MNCN)

Madrid: 2 ejemplares (A. Sanz leg.) (Col. MNCN) VK47

Madrid: Casa de Campo: VII, 1 ejemplar (Col. MNCN)

$\begin{array}{ll}\text { Madrid: Casa de Campo: VII, } 1 \text { ejemplar (Col. MNCN) } & \text { VK37 } \\ \text { Madrid: Fuencarral: } 1 \text { ejemplar (G. Schramm leg.) (Col. MNCN) } & \text { VK48 }\end{array}$

$\begin{array}{lll}\text { VK43 Madrid: Moncloa: } 1 \text { ejemplar (Col. MNCN) } & \text { VK37 }\end{array}$

VK43 Mejorada del Campo: 27-VI-1992, 1 ejemplar (F. A. Montes leg.)

(Col. MNCN) VK57

$\begin{array}{ll}\text { (Col. MNCN) } & \text { VK57 } \\ \text { Montarco: } 6 \text { ejemplares (Arias leg.) (Col. MNCN) } & \text { VK56 }\end{array}$

Montarco: VI-1908, 4 ejemplares (Arias leg.) (Col. MNCN) VK56

Móstoles: 1 ejemplar (J. Ardois leg ) (Col. MNCN)

Villaviciosa: 6 ejemplares (J. Ardois leg.) (Col. MNCN) VK26

Zonitis (Zonitis) immaculata (Olivier, 1789)

Alcalá: 2 ejemplares (Col. MNCN)

El Escorial: 1 ejemplar (Sanz leg.) (Col. MNCN)

El Escorial: 11 ejemplares (Col. MNCN)

VK09

El Escorial: 8 ejemplares (J. Lauffer leg.) (Col. MNCN) VK09

El Goloso: 11-VI-1989, 4 ejemplares (J.L. Zapata leg.) (Col. JLZ) VK48

Guadarrama: 22-VI-1991, 4 ejemplares (J.L. Zapata leg.) (Col. JLZ) VL00

Madrid: 1 ejemplar (Col. MNCN)

Madrid: 2 ejemplares (J. Ardois leg.) (Col. MNCN) VK47

VK76 Madrid: Moncloa: 1 ejemplar (G. Schramm leg.) (Col. MNCN) VK37

VK26 Puerto de Los Leones: 23-IV-1998, 1 ejemplar (F.A. Montes leg.) (Col. MNCN)

Vill. [Villaviciosa]: 1 ejemplar (Col. MNCN)

VL00

VK26

VK47

VK36

Apalus (Criolis) guerini (Mulsant, 1858)

Cerro del Telégrafo, Rivas: 15-V-1987 (R. Sánchez leg.) (Col. RS) 